\title{
An Analytical and Numerical Study of Internal Gravity Waves Forced by Isolated Topography
}

\section{Lidia Nikitina}

\author{
A thesis submitted to \\ the Faculty of Graduate Studies and Research \\ in partial fulfillment of \\ the requirements for the degree of \\ Master of Science
}

School of Mathematics and Statistics Ottawa-Carleton Institute of Mathematics and Statistics

Carleton University

Ottawa, Ontario, Canada

August, 2008

Copyright (C)

2008 Lidia Nikitina 


$\begin{array}{ll}\begin{array}{l}\text { Library and } \\ \text { Archives Canada }\end{array} & \begin{array}{l}\text { Bibliothèque et } \\ \text { Archives Canada }\end{array} \\ \begin{array}{l}\text { Published Heritage } \\ \text { Branch }\end{array} & \begin{array}{l}\text { Direction du } \\ \text { Patrimoine de l'édition }\end{array} \\ \begin{array}{l}\text { 395 Wellington Street } \\ \text { Ottawa ON K1A 0N4 } \\ \text { Canada }\end{array} & \begin{array}{l}\text { 395, rue Wellington } \\ \text { Ottawa ON K1A 0N4 } \\ \text { Canada }\end{array}\end{array}$

Your file Votre référence ISBN: 978-0-494-44135-0 Our file Notre référence ISBN: 978-0-494-44135-0

NOTICE:

The author has granted a nonexclusive license allowing Library and Archives Canada to reproduce, publish, archive, preserve, conserve, communicate to the public by telecommunication or on the Internet, loan, distribute and sell theses worldwide, for commercial or noncommercial purposes, in microform, paper, electronic and/or any other formats.

The author retains copyright ownership and moral rights in this thesis. Neither the thesis nor substantial extracts from it may be printed or otherwise reproduced without the author's permission.
AVIS:

L'auteur a accordé une licence non exclusive permettant à la Bibliothèque et Archives Canada de reproduire, publier, archiver, sauvegarder, conserver, transmettre au public par télécommunication ou par l'Internet, prêter, distribuer et vendre des thèses partout dans le monde, à des fins commerciales ou autres, sur support microforme, papier, électronique et/ou autres formats.

L'auteur conserve la propriété du droit d'auteur et des droits moraux qui protège cette thèse. $\mathrm{Ni}$ la thèse ni des extraits substantiels de celle-ci ne doivent être imprimés ou autrement reproduits sans son autorisation.
In compliance with the Canadian Privacy Act some supporting forms may have been removed from this thesis.

While these forms may be included in the document page count, their removal does not represent any loss of content from the thesis.
Conformément à la loi canadienne sur la protection de la vie privée, quelques formulaires secondaires ont été enlevés de cette thèse.

Bien que ces formulaires aient inclus dans la pagination, il n'y aura aucun contenu manquant.

\section{Canada}




\begin{abstract}
This thesis describes an analytical and numerical study of internal gravity waves in the atmosphere or ocean. Gravity waves result from the effects of gravitational forces and density stratification, and are frequently generated by fluid flow over topography (mountain). Previous related studies considered gravity waves generated by imposing a boundary condition in the form of a horizontally periodic monochromatic function at the lower boundary of a rectangular domain. Here a horizontally localized forcing, such as a Gaussian function, is used to represent an isolated mountain.

The problem is governed by two small parameters that define the height and width of the mountain. Depending on their magnitude the problem can be linear or nonlinear. Analytical techniques including Laplace and Fourier transforms, multiple scaling and asymptotic expansions are used to obtain approximate solutions of the governing linear and nonlinear equations. The analytical results are compared with the results of numerical simulations.
\end{abstract}




\section{Acknowledgements}

I would like to express my deepest gratitude to my supervisor, Prof. Lucy Campbell and thank her for her permanent support and patience, for her wisdom and kindness, for her high-level of professionalism both in research and teaching, for the great time I spent at the School of Mathematics and Statistics during my work over my Master's thesis. 


\section{Contents}

Abstract $\quad$ i

Acknowledgements ii

List of figures vii

1 Introduction 1

1.1 Study of gravity waves . . . . . . . . . . . . . . . . . . 1

1.2 Motivation for this study and overview of the thesis . . . . . . . . . 4

$\begin{array}{lll}2 & \text { Governing equations } & 8\end{array}$

2.1 Fluid mechanics . . . . . . . . . . . . . . . . . 8

2.2 Conservation laws . . . . . . . . . . . . . . . . . . . . 9

Conservation of mass . . . . . . . . . . . . . . . 10

Conservation of momentum . . . . . . . . . . . . . . 11

The first law of thermodynamics . . . . . . . . . . . . . . . 14

Specific heat and the equation of state ............. 15

Conservation of energy . . . . . . . . . . . . . . . . 17

2.3 The Boussinesq approximation . . . . . . . . . . . . . . . 20

The basic approximation . . . . . . . . . . . . . . 21 
2.4 The streamfunction and the vorticity . . . . . . . . . . . . 27

2.5 Some important parameters . . . . . . . . . . . . . . . . . . . 29

3 Gravity waves in the atmosphere $\quad 34$

3.1 Gravity waves: governing equations . . . . . . . . . . . . . . 34

Vorticity equation . . . . . . . . . . . . . . . . . . 37

3.2 The Taylor-Goldstein equation . . . . . . . . . . . . . . . . 4 40

3.3 Normal mode solution and the Taylor-Goldstein equation . . . . . . . . 41

The solution of the Taylor-Goldstein equation for the case $\bar{u}=$ constant 42

3.4 Stability of stratified shear flows . . . . . . . . . . . . 48

4 Exact solutions of the Taylor-Goldstein equation 51

4.1 Solution of the Taylor-Goldstein equation: the general case, using the method of Frobenius . . . . . . . . . . . . . . . . 52

Solution of the Taylor-Goldstein equation for an arbitrary function $\bar{u}(z) \quad 52$

4.2 Solution of the Taylor-Goldstein equation: $\bar{u}$ is a linear function of $z$,

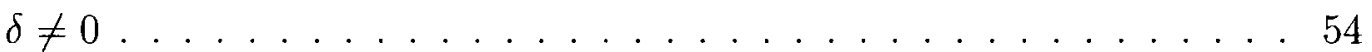

4.3 Solution of the Taylor-Goldstein equation: $\bar{u}$ is a linear function of $z$, $\delta=0 \ldots \ldots \ldots \ldots \ldots$

5 Gravity waves: solutions of the time-dependent equations with $k \neq 0 \quad 59$

5.1 Linear problem for monochromatic forced waves . . . . . . . . . . . . 60

5.2 Nonlinear solution . . . . . . . . . . . . . . . . . . 61

5.3 Wave packet forcing, $\mathrm{k} \neq 0, \delta=0 \ldots \ldots \ldots \ldots$

6 Gravity waves forced by an isolated mountain ( $k=0$ : analytical $\begin{array}{ll}\text { solution) } & 67\end{array}$

6.1 Steady state case . . . . . . . . . . . . . . . . . 68 
6.2 Time-dependent solution . . . . . . . . . . . . . . . . 75

6.3 Inner solution. Linear case . . . . . . . . . . . . . . . . 79

6.4 Nonlinear case . . . . . . . . . . . . . . . . . . . . . . . . . 81

6.5 Late-time solution . . . . . . . . . . . . . . . . . . 85

$\begin{array}{lll}7 & \text { Numerical study of gravity waves over an isolated topography } & 89\end{array}$

7.1 Description of the numerical methods . . . . . . . . . . 89 Horizontal discretization . . . . . . . . . . . . . . 91

Vertical discretization . . . . . . . . . . . . . . . 93

Non-uniform mesh for the critical layer . . . . . . . . . . . 94

7.2 Solutions for $k \neq 0$ (Campbell and Maslowe, 2003) . . . . . . 96

7.3 Results of the numerical simulation for the gravity wave over an isolated

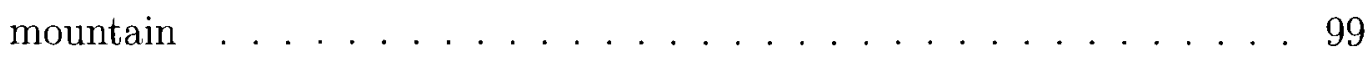

8 Conclusions $\quad 104$

8.1 Discussion of the analytical solutions $\ldots \ldots \ldots \ldots$

8.2 Discussion of the numerical solutions . . . . . . . . . . 106

$\begin{array}{ll}\text { A } & 108\end{array}$

Laplace transform . . . . . . . . . . . . . . 108

Inverse Laplace transform $\ldots \ldots \ldots$. . . . . . . . . . . . . . . .

$\begin{array}{ll}\text { B } & 115\end{array}$

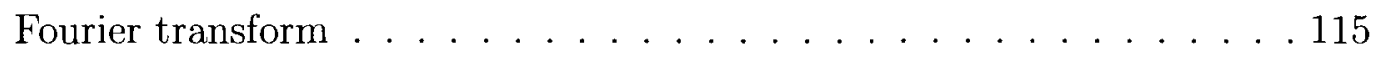

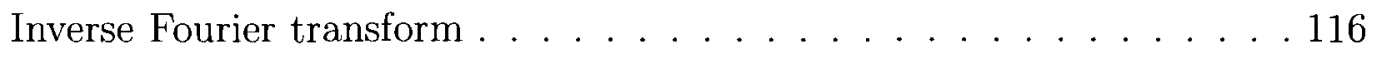

$\begin{array}{ll}\text { C Asymptotic and multiple scale analysis } & 120\end{array}$ 
D The Eliassen-Palm theorem and the mean flow evolution

D.1 Momentum flux and mean flow . . . . . . . . . . . . . . 122

D.2 The Eliassen-Palm theorem . . . . . . . . . . . . . 125

Bibliography 


\section{List of Figures}

3.1 Propagation of gravity waves. The gravity waves propagate with the phase speed $c$. The wave number vector $\mathbf{k}(k, m)$ points in the direction of the wave propagation. Lines of constant phase (wave front) are given by $\theta=k x+m z-\omega t=$ constant. The vector of the group velocity $\mathbf{c}_{\mathfrak{g}}$ is perpendicular to the wavenumber vector $\mathbf{k}$. The vectors $\mathbf{k}$ and $\mathbf{c}_{\mathbf{g}}$ have vertical components of opposite signs. . . . . . . . . . . . . . 47

5.1 Boundary conditions in the form of a wave packet $\psi=e^{-\mu^{2} x^{2}} e^{i k x}$ with wavenumber $k$ and length $\sim \mu^{-1} \ldots \ldots \ldots \ldots \ldots 6$

6.1 Boundary conditions in the form of an isolated mountain $\psi=e^{-\mu^{2} x^{2}}$ with length $\sim \mu^{-1} \ldots \ldots \ldots \ldots \ldots \ldots \ldots$

6.2 Contours of the streamfunction $\psi(X, z)(6.39)$ with the boundary condition $A(\mu x)=e^{-\mu^{2} x^{2}}, N=2.0, \mu=0.2$ and $\delta=0.1 \ldots \ldots 88$

7.1 Wave packet forcing: $e^{-\mu^{2} x^{2}} e^{2 i x}$. Horizontal velocity perturbation as a function of $x$ and $z$ at $t=10$. Parameters: $\varepsilon=0.05, \mu=0.2, N=2.0$. Regions where $u>0$ are shaded. . . . . . . . . . . . 97

7.2 Vertical variation of the mean vertical momentum flux $F(z, t)$ for the wave packet forcing $e^{-\mu^{2} x^{2}} e^{2 i x}$. The result obtained at $t=50$. Parameters: $\varepsilon=0.05, \mu=0.2, N=2.0$ 
7.3 Variation of the jump in the momentum flux with $t$. Comparison of the results obtained with the wave-packet forcing $e^{-\mu^{2} x^{2}} e^{2 i x}$ for the linear case $\varepsilon=0$ (dashed line) and for the nonlinear case $\varepsilon=0.05$ (solid line). $\mu=0.2, N=2.0$

7.4 Developing of the gravity wave packet forced by isolated mountain $e^{-\mu^{2} x^{2}}$. Contour plots of streamfunction $\psi(X, z, t)$ obtained at $t=2$ (a), $t=5$ (b), $t=10$ (c), $t=40$ (d) for the nonlinear case when $\varepsilon=0.2$. Parameters: $\mu=0.2, N=2.0 \ldots \ldots \ldots$. . . . . . . 100

7.5 Momentum flux as a function of z. $t=10$. Gravity waves forced by an isolated mountain $e^{-\mu^{2} x^{2}}$. Parameters: $\mu=0.2, N=2.0, t=50.0$. the momentum flux is plotted for the nonlinear case (solid line) where $\varepsilon=0.2$ and for the linear case where $\varepsilon=0$ (dashed line) . . . . . . 101

7.6 Gravity wave packet forced by an isolated mountain $e^{-\mu^{2} x^{2}}$. Contour plots of streamfunction $\psi(X, z, t)$ for the case $\varepsilon=0.01$ (a) and $\varepsilon=0.2$ (b) The results are obtained at $t=50$. Parameters: $\mu=0.2, N=2.0 . .103$

A.1 Contour of integration in the complex plane for equation (A.8). . . . 114 


\section{Chapter 1}

\section{Introduction}

\subsection{Study of gravity waves}

A stably stratified fluid is one in which the fluid density increases with depth. A characteristic of a stably stratified fluid is the ability to support and propagate wave motions. The atmosphere is almost always stably stratified, and it is reasonable to assume that it always contains waves forced by buoyancy and gravity, and Coriolis effects. The waves that result from the Coriolis force, i.e. the rotation of the Earth, are generally large-scale with wavelengths that can be of the order of magnitude of the Earth's circumference. These waves are called planetary waves, or Rossby waves (after the Swedish meteorologist Carl-Gustaf Rossby (1898-1957)).

Gravity waves are oscillations that occur as a result of upward buoyancy forces and the restoring force of gravity. When gravity waves occur at the free surface of a liquid they are called surface gravity waves; these include gravity waves on an air-sea interface, for example, ocean waves generated by wind. In this thesis we shall focus on internal gravity waves which propagate within a continuously stratified fluid such as the atmosphere or ocean. We shall refer to these internal waves simply as 
gravity waves. The wavelengths of internal gravity waves vary from 10 to $1000 \mathrm{~km}$ with periods from 10 minutes up to several hours. The speed of the particles inside gravity waves in the atmosphere is about $30-50 \mathrm{~ms}^{-1}$. The frequency of gravity waves is generally much larger than the Coriolis frequency. So, we can neglect the effect of the Earth's rotation on the waves.

We cannot see atmospheric gravity waves; we can only see the effects of the waves in the atmosphere, for example in clouds, and we can feel their effect, for example as turbulence while flying in aircraft. Today, it is recognized that gravity waves play an essential role in atmospheric processes on all meteorological scales.

Although the characteristics of waves in stratified fluid had been known for many years, one of the pioneering works on the theory of gravity waves was written by Hines (1960). He used gravity wave theory to explain the origins of turbulence observed in the ionosphere. Since then there have been many studies of effects of gravity waves on the upper atmosphere and the general circulation of the atmosphere. Some of these are reviewed by Fritts (1984) and Nappo (2002). Gravity waves sources include thunderstorms, thermal effects and air flow over topography (mountains). Here we shall focus mainly on topographic or mountain waves.

Most theoretical studies of gravity waves have been based on the linear theory. The process of linearization divides the meteorological variables into slowly varying or stationary background parts and small first-order perturbations, which we take to be due to waves. In the middle and upper atmosphere, the background flow often approaches these conditions. The linearization process eliminates the interactions of waves with waves and the resultant transfers of energy, but simplifies the governing equations to the point where they can sometimes be solved analytically.

In this study we consider the dynamics of gravity waves forced by isolated topography, i.e. a mountain or a hill with a single peak. Density-stratified air flow over 
mountains may give rise to internal gravity waves which affect the general circulation in the atmosphere. Such waves are referred to as topographic or mountain waves. Theoretical studies of gravity wave propagation in stratified shear flows have helped us understand the mechanisms for topographic wave generation, propagation, and gravity-wave - mean flow interactions. By means of simplifications such as linearization, the governing equations can be simplified to cases where the equations can be solved analytically.

An important property of waves is their ability to transport energy. Gravity waves transport energy away from the disturbances that generate them (mountains, hills, thunderstorms) and act to distribute this energy through the atmosphere. The distribution of energy is more rapidly done by wave than by mean flow.

One of the mechanisms for energy and momentum transport by gravity waves is the critical-level interaction. Such interactions take place when gravity waves reach a level in the atmosphere where the background wind speed equals the wave phase speed, i.e. if the background flow velocity is $\bar{u}(z)$ and the wave takes the form $\phi(z) e^{i k(x-c t)}$, then the critical level is the level $z_{c}$ where $\vec{u}(z)=c$.

The critical level corresponds to a singularity in the governing linearized steadystate equations, as we shall see in section 4.3. The wave amplitude is discontinuous across the critical level. For a wave propagating upwards towards a critical level, the amplitude is zero above the critical level, which implies that the wave is "absorbed" by the mean flow at the critical level (Booker and Bretherton, 1967). In a nonlinear problem, there are wave-mean flow interactions, and the mean flow changes as a result of the wave absorption (Brown and Stewartson, 1982). Nonlinear numerical solutions (Campbell and Maslowe, 2003) show that this wave absorption takes place at an early time, but later wave reflection may occur. This was suggested by the earlier analytical study of Brown and Stewartson (1982). There has been considerable debate in the 
past few decades about the mechanisms for the absorption or reflection of the waves.

In this study, analytic and numerical methods are used to examine the nonlinear dynamics of gravity waves forced by an isolated mountain. The topographic gravity waves take the form of a packet, localized in the horizontal direction and comprising a continuous spectrum of horizontal wavenumbers centered at zero. The wave packet propagates upwards in a density-stratified shear flow and reaches a critical level, where the horizontal mean wind is the same as the wave phase speed. The governing linear and nonlinear time-dependent equations are solved to study gravity wave propagation and the nonlinear interactions that take place between the wave packet and the mean wind in the vicinity of the critical level.

The motivation for the thesis is discussed in section 1.2 and an overview of the thesis is given.

\subsection{Motivation for this study and overview of the thesis}

Campbell and Maslowe's (2003) numerical study dealt with gravity waves critical levels where the gravity waves take the form of a wave packet localized in the horizontal direction. They focused on the specific case where the wave packet is generated by a lower boundary condition of the form $e^{-\mu^{2} x^{2}} \cos k x$, where $\mu$ and $k$ are constants, $k$ corresponds to a horizontal wavenumber, and $\mu \ll 1$. This is a more realistic representation of gravity waves in the real atmosphere or ocean than in previous studies which used monochromatic periodic forcing functions with no amplitude modulation such as $\cos k x$.

Campbell and Maslowe (2003) found that with a wave packet forcing the wave - 
mean-flow interactions differ from what is observed with a monochromatic forcing. With a monochromatic forcing they saw that the waves are absorbed at the critical level at early time and reflected at later time, as predicted by the analytic studies of Brown and Stewartson (1982). Campbell and Maslowe (2003) found that with the wave packet forcing, wave absorption continues to late time with no reflections. There was a flux of wave momentum in the horizontal direction which allowed the waves to continue being absorbed at late time. Campbell and Maslowe (2003) suggested that the mechanisms for this could be better understood and explained by carrying out an analytic study and deriving approximate solutions. However, the process of deriving approximate analytic solutions to the nonlinear problem with wave packet forcing is extremely complicated. In this study we derive approximate analytic solutions for the special case where the wave packet forcing comprises a spectrum of horizontal wavenumbers centered at $k=0$, so that the lower boundary condition takes the form $\psi=A(\mu x), \mu \ll 1$, which varies slowly with respect to $x$ and goes to zero as $x \rightarrow \pm \infty$. An example of such a function is the Gaussian profile $A(\mu x)=e^{-\mu^{2} x^{2}}$. With $k=0$ the derivation of an approximate analytic solution is more straightforward and so we are able to obtain some insight into the nonlinear evolution of the wave packet.

An overview of the thesis is as follows.

Chapters 2-4 provide a lot of background information to help the reader follow the solutions derived in the later chapters. The original work of the author of the thesis starts in Chapter 5.

In Chapter 2 we review fundamental concepts and laws of fluid mechanics which will be used in other chapters and give an outline of the derivation of the equations of fluid mechanics based on conservation of mass, momentum and energy.

In Chapter 3 the governing equations for gravity wave propagation are derived. Special attention is paid to the normal mode solution which assumes that the waves 
are periodic in space and time, and the Taylor-Goldstein equation which is derived using the normal mode approach.

In Chapter 4 we consider some analytical solutions of the Taylor-Goldstein equation for certain special configurations of the basic state. Also, the influence of the critical layer on gravity wave propagation is discussed.

Chapters 2-4 follow basic textbooks on fluid dynamics such as Landau and Lifshitz (1953), Batchelor (1967), Kundu and Cohen (2004), and also classic monographs and papers on gravity wave propagation such as Booker and Bretherton (1967), Baines (1989), and Nappo (2002). Throughout the thesis we consider two dimensional configurations defined in terms of a horizontal and a vertical coordinate, and a time variable.

Chapter $\mathbf{5}$ is devoted to the time-dependent problem in which the waves are assumed to be periodic in the horizontal direction only, but the wave amplitude varies in time and in the vertical direction. The governing time-dependent equations are linearized and approximate analytical solutions are derived for the two cases: a wave forced by a monochromatic horizontally-periodic boundary condition $e^{i k x}$, and a wave packet forced by a horizontally-localized boundary condition like a mountain range $A(\mu x) e^{i k x}$. In the monochromatic case the solution was first derived by Booker and Bretherton (1967) and we describe it here. For the wave packet case numerical solutions for the linear and nonlinear problem were obtained by Campbell and Maslowe (2003). In this thesis an approximate analytic solution for the linear case is derived using Booker and Bretherton's (1967) monochromatic solution as a starting point. The details of the solution procedure are given in the appendix.

Chapter 6 also presents the original research of the thesis' author. A timedependent analytical solution is derived for the governing equations for the case when boundary conditions are in the form of an isolated mountain, $A(\mu x) \cos k x$ with $k=0$. 
This study includes

- the solution of the governing linearized equation using the method of multiple scaling for the steady case and for the time-dependent case;

- a discussion of the nature of the critical-level interaction for the linear solution;

- the solution of the governing equations for an inner layer in the vicinity of a critical layer for both linear and nonlinear cases.

Chapter 7 is devoted to a numerical study of gravity waves forced by an isolated mountain. The numerical simulations make use of the numerical methods of Campbell and Maslowe (2001), and Campbell and Maslowe (2003). The computational code developed there is modified to suit the present problem. The results of the numerical simulations are compared with the approximate analytical solutions derived in Chapter 6. 


\section{Chapter 2}

\section{Governing equations}

\section{$2.1 \quad$ Fluid mechanics}

In this chapter we describe and derive the basic equations of fluid mechanics.

Fluid mechanics deals with the flow of fluids. Most substances exist in one of the two states: solid or fluid. An element of solid has a preferred shape. In contrast, a fluid does not have any preferred shape. Under the action of a shear force, the solid element assumes another shape. If the solid is perfectly elastic, it goes back to its preferred shape when the force is withdrawn. In contrast, a fluid deforms continuously under the action of a shear force, however small. Although solids and fluids behave very differently when subjected to shear stresses, they behave similarly under the action of compressive normal stresses. A solid can support both tensile and compressive normal stresses, and a fluid usually supports only compression stresses. Fluids again may be divided into two classes, liquids and gases. A gas always expands and occupies the entire volume of any container. In contrast, the volume of a liquid does not change very much, so that it cannot completely fill a large container. The properties of solids, liquids and gases are related to their molecular structure and 
forces between molecules.

A fluid, or any other substance, is composed of a large number of molecules in constant motion or undergoing collisions with each other. So, any substance is discontinuous at microscopic scales. There are two levels of studying a fluid: small scale, or microscopic, and large scale, or macroscopic consideration. It is possible to ignore the discrete molecular structure of matter and replace it by a continuous distribution. For the continuum or macroscopic approach to be valid, the size of the flow system must be much larger than the molecules and intermolecular distance. Here a fluid will be considered as continuous, because we are interested in the gross behaviour of the fluid. This consideration is known as a continuum hypothesis.

In this study we consider waves in the atmosphere and in the ocean, so the fluid under consideration could be either a gas or a liquid. The theory and properties of fluids are described in most fluid mechanics books. In the rest of this chapter we describe and derive the governing equations of fluid mechanics following Landau and Lifshitz (1953), Batchelor (1967), and Kundu and Cohen (2004).

\subsection{Conservation laws}

The study of fluid mechanics is based on the laws of conservation of mass, momentum, and energy. These laws can be stated in differential form, applicable at a point. They can also be stated in integral form, applicable to a volume. The equations of fluid mechanics are defined in terms of the fluid properties such as density $\rho$, pressure $p$ and velocity $\mathbf{u}$. We also define a time variable $t \geq 0$. In this thesis we define the equations in a rectangular system of coordinates $x, y$, and $z$ with unit vectors $\mathbf{i}, \mathbf{j}, \mathbf{k}$ respectively, but any other coordinate system could be assumed instead, e.g. spherical or cylindrical coordinate systems. In this section we give an overview of the derivation 
of the governing equations of fluid mechanics. Our derivation and notation follow that of Batchelor (1967) and Kundu and Cohen (2004).

\section{Conservation of mass}

To derive an equation for conservation of mass we consider some volume $V$ that is fixed in space. The total mass inside the volume will be

$$
\int_{V} \rho(x, y, z, t) d x d y d z=\int_{V} \rho d V
$$

if the density of the fluid occupying the volume is $\rho(x, y, z, t)$.

For conservation of mass the decrease in mass inside $V$ has to be balanced by the rate of mass flow out of the volume, i.e.

$$
\int_{V} \frac{\partial \rho}{\partial t} d V=-\int_{A} \rho u d A
$$

where $A$ is the surface of the volume $V$.

The surface integral on the right-hand side of equation (2.2) can be transformed into a volume integral by means of the divergence theorem, which gives

$$
\int_{A} \rho u d A=\int_{V} \nabla \cdot(\rho \mathbf{u}) d V
$$

Equation (2.1) then becomes

$$
\int_{V}\left[\frac{\partial \rho}{\partial t}+\nabla \cdot(\rho \mathbf{u})\right] d V=0
$$

Since this relation holds for any arbitrary volume $V$, it means that the integrand equals zero at any point: 


$$
\frac{\partial \rho}{\partial t}+\nabla \cdot(\rho \mathbf{u})=0
$$

assuming that $\rho$ and $\mathbf{u}$ are continuous and differentiable everywhere in $V$. This equation is called the continuity equation and expresses the differential form of the principle of conservation of mass.

A fluid is usually called incompressible if the density does not change with pressure. Liquids are almost incompressible. Although gases are compressible, for speeds $\leq$ $100 \mathrm{~ms}^{-1}$ (that is for Mach numbers $\leq 0.3$ ) the fractional change of absolute pressure in the flow is small (Batchelor, 1967). In this and several other cases the density changes in the flow are also small. In such a case the continuity equation reduces to the incompressible form

$$
\nabla \cdot \mathbf{u}=0
$$

\section{Conservation of momentum}

Consider a fluid element of fixed mass with a volume $d V$. According to Newton's second law the rate of change in momentum due to an external force is

$$
\mathbf{F}=\frac{D \mathbf{M}}{D t}
$$

where $\mathbf{M}$ is the momentum vector and $\mathbf{F}$ is the external force. For a unit volume

$$
\mathbf{M}=\rho \frac{D \mathbf{u}}{D t}
$$


The notation $\frac{D}{D t}$ refers to the total derivative:

$$
\frac{D}{D t}=\frac{\partial}{\partial t}+u_{i} \frac{\partial}{\partial x_{i}}
$$

where repeated index $i$ implies summation over $i=1,2,3$.

Any volume is under the action of two kinds of forces; body forces and surface forces. Body forces act on the volume of a body without physical contact; they are external forces produced by force fields, such as gravitational fields or electro-magnetic fields. In our case we will consider a situation where there is a gravitational force. Per unit volume this force is $\rho \mathbf{g}$, where $\mathbf{g}=-g \mathbf{k}$ is the acceleration due to gravity.

Surface forces are defined in terms of a stress tensor which can be defined using the notation $\left\{\tau_{i j}\right\}$, where the first index specifies the orientation of the surface and the second index denotes the direction of the force applied to the surface. So, for a volume $V$, the rate of change of momentum is

$$
\frac{D}{D t} \int_{V} \rho u_{i} d V=\int_{V} \rho g_{i} d V+\int_{A} \tau_{i j} d A_{j}
$$

where $i=1,2,3$ in a three-dimensional configuration and the repeated index $j$ in the last term implies that we are summing over $j=1,2,3$. The differential form can be obtained by transforming the surface integral on the right-hand side of equation to a volume integral by means of the divergence theorem (2.3). Equation (2.10) then becomes

$$
\int_{V}\left[\rho \frac{D u_{i}}{D t}-\rho g_{i}-\frac{\partial \tau_{i j}}{\partial x_{j}}\right] d V=0 .
$$

Since the relations holds for any arbitrary volume, it means that the integrand equals zero at any point, which gives: 


$$
\rho \frac{D u_{i}}{D t}=\rho g_{i}+\frac{\partial \tau_{i j}}{\partial x_{j}}
$$

This equation is known as Cauchy's equation of motion.

For a moving fluid, Cauchy's stress tensor consists of two parts:

$$
\tau_{i j}=-p \delta_{i j}+\sigma_{i j}, \quad i, j=1,2,3
$$

where $p$ is the pressure, and $\delta_{i j}$ is the Kronecker delta defined as:

$$
\delta_{i j}= \begin{cases}1, & \text { if } i=j \\ 0, & \text { otherwise }\end{cases}
$$

In a fluid, the first part $-p \delta_{i j}$ corresponds to pressure, and the second part $\sigma_{i j}$ describes viscous friction. For an incompressible fluid, the viscous term is proportional to the velocity gradients, i.e.

$$
\sigma_{i j}=\mu\left(\frac{\partial u_{i}}{\partial x_{j}}+\frac{\partial u_{j}}{\partial x_{i}}\right)+\lambda \delta_{i j} \frac{\partial u_{m}}{\partial x_{m}}
$$

where $\mu$ and $\lambda$ are called first and second coefficients of dynamic viscosity, respectively. As before repeated indices, i.e. $m$ in the last term, imply summation over $m=1,2,3$. A simplifying assumption that is frequently made is the Stoke's assumption $\lambda=-\frac{2}{3} \mu$. Substituting this into Cauchy's equation gives

$$
\rho \frac{D u_{i}}{D t}=-\frac{\partial p}{\partial x_{j}}+\rho g_{i}+\frac{\partial}{\partial x_{j}}\left[\mu\left(\frac{\partial u_{i}}{\partial x_{j}}+\frac{\partial u_{j}}{\partial x_{i}}\right)-\frac{2}{3} \mu \nabla \cdot \mathbf{u} \delta_{i j}\right]
$$

which is known as the Navier-Stokes equation. For an incompressible fluid $\nabla \cdot \mathbf{u}=0$ 
and we so can rewrite the momentum equation in the form:

$$
\rho \frac{D u_{i}}{D t}=-\frac{\partial p}{\partial x_{i}}+\rho g_{i}+\mu \nabla^{2} u_{i}
$$

or in the vector form as

$$
\rho \frac{D \mathbf{u}}{D t}=-\nabla p+\rho \mathbf{g}+\mu \nabla^{2} \mathbf{u}
$$

For an inviscid fluid $\mu=0$ and we obtain Euler's equation

$$
\rho \frac{D \mathbf{u}}{D t}=-\nabla p+\rho \mathbf{g}
$$

\section{The first law of thermodynamics}

The first law of thermodynamics states that the total energy of a system is conserved. It states that

$$
Q+W=\Delta e
$$

where $Q$ is the heat added to the system, $W$ is the work done on the system, and $\Delta e$ is the increase of internal energy of the system. Frictionless quasi-static processes, carried out at an extremely slow rate so that the system is at all times in equilibrium with the surroundings, are called reversible processes. The most common type of reversible work in fluid flow is an expansion or contraction of the boundaries of the fluid element. The work done by the body force per unit mass in reversible process is $-p d V$, where $d V$ is the increase of $V$. The first law (2.19) for a reversible process then becomes

$$
d e=d Q-p d V
$$




\section{Specific heat and the equation of state}

In a simple system composed of a single component only, two independent properties completely determine the state of the system. This means that any fluid properties can be determined if two properties are known, e.g. if we know the specific volume $V$ and the temperature $T$ we can find the pressure $p$. We can write relations such as

$$
p=p(V, T)
$$

which is a thermal equation of state, and

$$
e=e(p, T)
$$

which is a caloric equation of state. Such relations are called equations of state. For more complicated systems composed of more than one physical component, the two properties are not enough to completely determine the state. For example, for sea water containing dissolved salt, the density is a function of the three variables, salinity, temperature, and pressure.

The enthalpy of a fluid is a thermodynamic property defined in terms of the internal energy $e$, the pressure $p$ and the volume $V$ by the equation

$$
h=e+p V
$$

For a single-component system, the specific heats at constant pressure and constant volume are defined as

$$
C_{p}=\left(\frac{\partial h}{\partial T}\right)_{p}
$$




$$
C_{v}=\left(\frac{\partial e}{\partial T}\right)_{V} .
$$

Here, equation (2.24) means that we consider $h$ as a function of $p$ and $T$, and find the partial derivative of $h$ with respect to $T$, keeping $p$ constant. Equation (2.25) means that we find the partial derivative with respect to $T$, keeping $V$ constant.

A perfect gas is defined as one that obeys the thermal equation of state,

$$
p=\rho R T
$$

where $p$ is the pressure, $\rho$ is the density, $T$ is the absolute temperature, and $R$ is the gas constant. The value of the gas constant depends on the molecular mass $m$ of the gas according to a relation

$$
R=R_{u} / m,
$$

where

$$
R_{u}=8314.36 \mathrm{Jkmol}^{-1} \mathrm{~K}^{-1}
$$

is the universal gas constant. For example, the molecular mass for dry air is $m=$ $28.966 \mathrm{~kg} / \mathrm{kmol}$, for which equation (2.27) gives

$$
R=287 \mathrm{Jkg}^{-1} \mathrm{~K}^{-1}
$$

The gas constant is related to the specific heats of the gas through the relation

$$
R=C_{p}-C_{V}
$$

where $C_{p}$ is the specific heat at constant pressure and $C_{V}$ is the the specific heat at 
constant volume. The ratio of specific heats of a gas

$$
\gamma=\frac{C_{p}}{C_{V}}
$$

is a constant. For air at ordinary temperatures, $\gamma \approx 1.4$.

A process is called adiabatic if it takes place without the addition of heat. A process is called isentropic if it is adiabatic and frictionless, for then the entropy of the fluid does not change. The isentropic flow of a perfect gas with constant specific heats obeys the relation

$$
\frac{p}{\rho^{\gamma}}=\text { const. }
$$

Using the equation of state $p=\rho R T$, it follows that the temperature and density change during an isentropic process from state 1 to a state 2 according to

$$
\frac{T_{1}}{T_{2}}=\left(\frac{p_{1}}{p_{2}}\right)^{(\gamma-1) / \gamma}
$$

and

$$
\frac{\rho_{1}}{\rho_{2}}=\left(\frac{p_{1}}{p_{2}}\right)^{1 / \gamma}
$$

\section{Conservation of energy}

To define an equation for the conservation of energy we first consider Cauchy's equation (2.12) and multiply each $i$-component of the equation by $u_{i}$, the $i$-component of the velocity:

$$
\rho \frac{D\left(\frac{1}{2} u_{i}^{2}\right)}{D t}=\rho g_{i} u_{i}+u_{i} \frac{\partial \tau_{i j}}{\partial x_{j}}
$$

Equation (2.35) is called the mechanical energy equation. The term on the left-hand side is the derivative of the kinetic energy $E=\frac{1}{2} \rho u_{i}^{2}$. We can rewrite the second term 
on the right-hand side as

$$
u_{i} \frac{\partial \tau_{i j}}{\partial x_{j}}=\frac{\partial}{\partial x_{j}}\left(u_{i} \tau_{i j}\right)-\tau_{i j} \frac{\partial u_{i}}{\partial x_{j}}
$$

and rewrite the mechanical energy equation in the form

$$
\rho \frac{D E}{D t}=\rho g_{i} u_{i}+\frac{\partial u_{i} \tau_{i j}}{\partial x_{j}}+p(\nabla \cdot \mathbf{u})-\phi
$$

where the first term on the right-hand side is the rate of work done by the body force (here it is the gravitational force), the second term is the work done by surface forces, the third term is a rate of work done by volume expansion due to change in the pressure, and $\phi$ is a rate of viscous dissipation:

$$
\phi=\mu\left[\frac{1}{4}\left(\frac{\partial u_{i}}{\partial x_{j}}+\frac{\partial u_{j}}{\partial x_{i}}\right)^{2}-\frac{2}{3}(\nabla \cdot \mathbf{u})^{2}\right]
$$

For a fixed volume $V$ we can write the mechanical energy equation in integral form as

$$
\int_{V}\left[\frac{\partial E}{\partial t}+\frac{\partial}{\partial x_{j}}\left(u_{i} E\right)\right] d V=\int_{V}\left[\rho g_{i} u_{i}+\frac{\partial u_{i} \tau_{i j}}{\partial x_{j}}+p(\nabla \cdot \mathbf{u})-\phi\right] d V
$$

Making use of the divergence theorem we get

$$
\frac{d}{d t} \int_{V} E d V+\int_{A} E \overline{\mathbf{u}} d A=\int_{V} \rho \mathbf{g} \mathbf{u} d V+\int_{A} u_{i} \tau_{i j} d A_{j}+\int_{V} p(\nabla \cdot \mathbf{u}) d V-\int_{V} \phi d V
$$

The first term on the right-hand side is the rate of change of kinetic energy and the second term is the rate of outflow across the boundary of the volume.

In fluid flow where there are temperature variations we need an additional principle 
for energy conservation taking thermodynamics into account. We make use of the first law of thermodynamic. The total energy per unit volume is the sum of the internal energy $e$ and the kinetic energy $E$.

For a volume $V$, the first law of the thermodynamics states that the rate of a change of total energy is the sum of the rate of work done by body forces and surface forces and the rate of heat flux through a surface of the volume. This can be written as

$$
\int_{V} \rho \frac{D\left(\frac{1}{2} u_{i}^{2}+e\right)}{D t} d V=\int_{V} \rho g_{i} u_{i} d V+\int_{A} \tau_{i j} u_{i} d A-\int_{A} q_{i} d A
$$

where vector $\mathbf{q}$ is the heat flux through a unit area. Using the divergence theorem and taking into account that the relation holds for any arbitrary volume, we can rewrite the equation (2.41) in the form:

$$
\rho \frac{D\left(\frac{1}{2} u_{i}^{2}+e\right)}{D t}=\rho g_{i} u_{i}+\frac{\partial}{\partial x_{j}}\left(\tau_{i j} u_{i}\right)-\frac{\partial q_{i}}{\partial x_{j}} .
$$

This is the first law of thermodynamics in differential form. The term on the left-hand side represents the rate of change of total energy (internal energy together with kinetic energy) and the first term on the right-hand side represents a potential energy. If we subtract the mechanical energy equation (2.37) from the equation for the first law of thermodynamics (2.42) we obtain:

$$
\rho \frac{D e}{D t}=-p(\nabla \cdot \mathbf{u})+\phi-\frac{\partial q_{i}}{\partial x_{i}}
$$

This is called the thermal energy equation.

The set of equations (2.5), (2.15) and (2.43) are used as the starting point for studies of fluid dynamics. The problem of deriving an exact solution to these equations 
remains open. However, under certain simplifying assumptions approximate solutions can be derived. An approximation that is often used for studying density stratified flows and internal gravity waves is the Boussinesq approximation, which is described in the next section.

\subsection{The Boussinesq approximation}

For flows satisfying certain conditions, Boussinesq in 1903 suggested that the density changes in the fluid can be neglected except in the gravity term where $\rho$ is multiplied by $\mathbf{g}$. The Boussinesq approximation is often used to simplify the equations governing fluid motion in order to facilitate both theoretical analysis and numerical computation. A lot of problems have been investigated on the basis of the Boussinesq equations, with good correlation between theoretical results and experimental measurements.

In fluid dynamics, the Boussinesq approximation is used in the field of buoyancydriven flow. It states that density differences are sufficiently small to be neglected, except where they appear in terms multiplied by $\mathrm{g}$, the acceleration due to gravity. The essence of the Boussinesq approximation is that the difference in inertia is negligible but gravity is sufficiently strong to make the specific weight appreciably different. Boussinesq flows are common in nature (such as atmospheric fronts, oceanic circulation, downhill winds), industry (dense gas dispersion, fume cupboard ventilation), and the built environment (natural ventilation, central heating). The approximation is accurate for many such flows, and makes the mathematics and physics simpler. The approximation is well-suited for studies of internal gravity waves. The governing equations for the configuration we shall study in this thesis are based on the Boussinesq approximation.

The Boussinesq approximation is summarized by two statements (see, e.g. Spiegel 
and Veronis (1960)):

- The fluctuations in density result principally from thermal effects.

- In the equations for the rate of change of momentum and mass, density variations can be neglected except when they are coupled to the gravitational acceleration in the buoyancy force.

\section{The basic approximation}

In this section we derive the basic Boussinesq approximation following the derivation given by Spiegel and Veronis (1960). We consider a three-dimensional configuration defined by rectangular coordinates $x, y, z$ and time $t$. Let $f$ represent any one of the state variables: density $\rho$, temperature $T$, or pressure $p$. We express each of these variables in the form:

$$
f(x, y, z, t)=f_{0}(z)+f^{\prime}(x, y, z, t)
$$

where $f_{0}$ is the horizontal and time average of $f$ and $f^{\prime}$ is any departure of the state variable from the average value. The scale height is

$$
D_{f}=\left|\frac{1}{f_{0}} \frac{d f_{0}}{d z}\right|^{-1}
$$

and defines the rate at which $f_{0}$ changes in the vertical direction. The basic condition for the Boussinesq approximation is that the fluid is confined to a layer whose thickness, $d$, is much less than the smallest scale height, $D_{f}$. So, if $D=\left(D_{f}\right)_{\min }$, then

$d<<D$. This means that changes in the fluid with height are not large and can be neglected to some extent. In this layer the temperature is $T=T_{0}+T^{\prime}$, where $T_{0}$ is a mean temperature value, $T^{\prime}<<T_{0}$, density $\rho=\rho_{0}+\rho^{\prime}$, where $\rho_{0}$ is constant, and 
$\rho^{\prime}<<\rho_{0}$, and the pressure is $p=p_{0}+p^{\prime}$, where $p_{0}$ is a function of the vertical variable $z$

$$
p=-\rho_{0} g z+p^{\prime}
$$

where $z$ is directed upward.

Let us consider how to simplify the equations of motions (2.5), (2.15) and (2.43) using the Boussinesq approximation.

The continuity equation under the Boussinesq approximation

With the density written as $\rho=\rho_{0}+\rho^{\prime}$ the continuity equation (2.5)

$$
\frac{\partial \rho}{\partial t}+\nabla \cdot(\rho \mathbf{u})=0
$$

can be written as

$$
\frac{\partial\left(\rho_{0}+\rho^{\prime}\right)}{\partial t}+\left(\rho_{0}+\rho^{\prime}\right) \nabla \cdot \mathbf{u}+\mathbf{u} \cdot \nabla\left(\rho_{0}+\rho^{\prime}\right)=0
$$

Under the Boussinesq approximation $\partial \rho / \partial t=0$, perturbations $u$ and $\rho^{\prime}$ are so small relative to the basic flow quantities $\rho$ and $u$ that their second order products can be neglected. The continuity equation can thus be approximated by

$$
\nabla \cdot \mathbf{u}=0
$$

\section{The momentum equation under the Boussinesq approximation}

The Navier-Stokes equation (2.15) for a viscous liquid can be written as

$$
\frac{\partial \mathbf{u}}{\partial t}+(\mathbf{u} \cdot \nabla) \mathbf{u}=-\frac{\nabla p}{\rho}+\mathbf{g}+\frac{\mu}{\rho} \nabla^{2} \mathbf{u}+\frac{\mu}{3 \rho} \nabla(\nabla \cdot \mathbf{u})
$$


where $\mu$ is the coefficient of kinematic viscosity. Here $\mu$ is taken to be constant. According to the continuity equation under the Boussinesq approximation (2.49), $\nabla \cdot \mathbf{u}=0$, so the last viscous term on the right hand side of equation (2.50) is zero. Let us consider a perfect gas for which the density $\rho$ is a function of two variables: $T$ and $p$, and write $p$ and $\rho$ each as a sum of a reference value and a perturbation:

$$
\begin{aligned}
& p=p_{0}(z)+p^{\prime}(x, y, z, t), \\
& \rho=\rho_{0}(z)+\rho^{\prime}(x, y, z, t) .
\end{aligned}
$$

Substituting these expressions into the momentum equation gives

$$
\left(\rho_{0}+\rho^{\prime}\right)\left(\frac{\partial \mathbf{u}}{\partial t}+(\mathbf{u} \nabla) \mathbf{u}\right)=-\nabla p_{0}-\nabla p^{\prime}+\rho_{0} \mathbf{g}+\rho^{\prime} \mathbf{g}+\mu \nabla^{2} \mathbf{u}
$$

According to (2.46) the reference pressure $p_{0}$ is a function of $z$ and $\nabla p_{0}=-\rho_{0}$ g. So, the second and third terms on the right-hand side of (2.53) cancel each other and we have:

$$
\left(1+\frac{\rho^{\prime}}{\rho_{0}}\right)\left(\frac{\partial \mathbf{u}}{\partial t}+(\mathbf{u} \nabla) \mathbf{u}\right)=-\frac{\nabla p^{\prime}}{\rho_{0}}+\frac{\rho^{\prime}}{\rho_{0}} \mathbf{g}+\frac{\mu}{\rho_{0}} \nabla^{2} \mathbf{u}
$$

On the left-hand side the term $\rho^{\prime} / \rho_{0}$ can be neglected, because $\rho^{\prime} / \rho_{0}<<1$, but it should not be neglected on the right-hand side because of the Boussinesq assumption that the density variation can be neglected everywhere except in the gravitational term in the momentum equation. If we neglect it in the gravitational term there would be no effects of gravity in the equation. So, the momentum equation in the Boussinesq approximation has the form:

$$
\frac{\partial \mathbf{u}}{\partial t}+(\mathbf{u} \nabla) \mathbf{u}=-\frac{\nabla p^{\prime}}{\rho_{0}}+\frac{\rho^{\prime}}{\rho_{0}} \mathbf{g}+\nu \nabla^{2} \mathbf{u}
$$


where $\nu=\mu / \rho_{0}$ is called the kinematic viscosity.

\section{The heat equation under the Boussinesq approximation}

According to the thermal heat equation (2.43)

$$
\rho \frac{D e}{D t}=-\nabla \cdot \mathbf{q}-p(\nabla \mathbf{u})+\phi
$$

where $e$ is the internal energy, the first term on the right-hand side is the heat flux, the second term is the work done by volume expansion, and $\phi$ is the viscous term. Under the Boussinesq approximation, we have

$$
-p(\nabla \mathbf{u})=\frac{p}{\rho} \frac{D \rho}{D t} \simeq \frac{p}{\rho}\left(\frac{\partial \rho}{\partial T}\right)_{p} \frac{D T}{D t}
$$

Now under the Boussinesq approximation $\nabla \cdot \mathbf{u}$ was approximated by zero in the continuity equation (2.49), but we can not justify setting it to zero in the energy equation (2.56). Although $\nabla \cdot \mathbf{u}$ is small, when it is multiplied by $p$ it is not negligible compared with the other terms in the equation. The continuity equation states that

$$
-\nabla \cdot \mathbf{u}=\frac{1}{\rho} \frac{D \rho}{D t}<<1
$$

but

$$
-p \nabla \cdot \mathbf{u}=\frac{p}{\rho} \frac{D \rho}{D t}
$$

which can be $O(1)$ if $p$ is large. In fact

$$
-p \nabla \cdot \mathbf{u}=\frac{p}{\rho} \frac{D \rho}{D t} \approx \frac{p}{\rho}\left(\frac{\partial \rho}{\partial T}\right)_{p} \frac{D T}{D t}
$$


For a perfect gas for which $p=\rho R T, C_{p}-C_{v}=R$, and $C_{p}=\left(\frac{\partial e}{\partial T}\right)_{V}$ we can estimate $p(\nabla \mathbf{u})$ as

$$
\begin{array}{r}
-p \nabla \cdot \mathbf{u}=\frac{p}{\rho} \frac{D}{D t}\left(\frac{p}{R T}\right) \approx \frac{p}{\rho} \frac{p}{R} \frac{D}{D t}\left(\frac{1}{T}\right)=\frac{p}{\rho} \frac{p}{R}\left(-\frac{1}{T^{2}}\right) \frac{D T}{D t}= \\
-R \rho \frac{D T}{D t}=-\left(C_{p}-C_{v}\right) \rho \frac{D T}{D t} .
\end{array}
$$

The heat equation (2.56) then becomes

$$
\rho \frac{D e}{D t}=-\nabla \cdot \mathbf{q}-\left(C_{p}-C_{v}\right) \rho \frac{D T}{D t}+\phi
$$

Taking into account that $e=C_{V} T$ we get

$$
C_{v} \rho \frac{D T}{D t}=-\nabla \cdot \mathbf{q}-\left(C_{p}-C_{v}\right) \rho \frac{D T}{D t}+\phi
$$

which simplifies to

$$
\rho C_{p} \frac{D T}{D t}=-\nabla \mathbf{q}+\phi
$$

Under the Boussinesq approximation viscous dissipation of energy is negligible. An assumption that is generally made is that $q$ satisfies Fourier's law of heat conduction

$$
\mathbf{q}=-\kappa \nabla T
$$

where $\kappa$ is the thermal conductivity. The heat equation then reduces to

$$
\rho C p\left(\frac{\partial}{\partial t}+\mathbf{u} \nabla\right) T^{\prime}=\nabla \cdot\left(\kappa \nabla T^{\prime}\right)
$$


or, if we consider $\kappa$ as a constant, this equation can be rewritten as

$$
\left(\frac{\partial}{\partial t}+\mathbf{u} \nabla\right) T^{\prime}=K \nabla^{2} T^{\prime}
$$

where $K=\kappa /\left(\rho C_{p}\right)$ is the coefficient of thermal diffusivity. For a steady flow with $D T / D t=0$ the heat equation takes the form of Laplace's equation

$$
\nabla^{2} T=0
$$

Finally we note that under the Boussinesq approximation we can rewrite the heat equation in terms of the density $\rho$ instead of the temperature $T$. This is because the equation of state under the Boussinesq assumptions can be approximated by a linear relation between the density $\rho$ and the temperature $T$. For a perfect gas

$$
\rho T=\frac{p}{R}
$$

We can write $\rho=\rho_{0}+\rho^{\prime}$ and $T=T_{0}+T^{\prime}$ as before, and linearize the equation about the reference state, assuming that the basic state satisfies the perfect gas relation.

$$
\left(\rho_{0}+\rho^{\prime}\right)\left(T_{0}+T^{\prime}\right)=\frac{p_{0}+p^{\prime}}{R} .
$$

Assuming that $\rho^{\prime}<<\rho_{0}$ and $T^{\prime}<<T_{0}$ we can neglect products of perturbations. So, we have a linear relation between $\rho^{\prime}$ and $T^{\prime}$

$$
\rho^{\prime}=\frac{\rho_{0}}{T_{0}} T^{\prime}
$$


or

$$
\rho-\rho_{0}=\frac{\rho_{0}}{T_{0}}\left(T-T_{0}\right)
$$

Because of this linear relation between $\rho$ and $T$ we can rewrite the heat equation (2.67) in terms of $\rho$

$$
\frac{D \rho}{D t}=K \nabla^{2} \rho
$$

In the rest of this thesis we will use the heat equation in this form.

\subsection{The streamfunction and the vorticity}

For an incompressible fluid flow (or a fluid flow under the Boussinesq approximation) the continuity equation takes the form $\nabla \cdot \mathbf{u}=0$. If the flow is two-dimensional, in rectangular coordinates, say $x$ and $z$, the continuity equation is

$$
u_{x}+w_{z}=0
$$

where $u$ and $w$ are the horizontal and vertical components of the velocity and the subscripts denote partial differentiation. This allows us to define a streamfunction by

$$
u=-\Psi_{z}, \quad w=\Psi_{x}
$$

which satisfies the continuity equation. Lines (or curves) with $\Psi=$ constant are called streamlines. Streamlines are curves that are everywhere tangent to the velocity vector $(u, w)$ at any time. For unsteady flows the velocity changes with time so the streamlines change with time too. Let $d \mathbf{s}=(d x, d z)$ be an element of an arc length along a streamline. Then

$$
\frac{d x}{u}=\frac{d z}{w}
$$


along a streamline. So,

$$
\frac{d x}{-\Psi_{z}}=\frac{d z}{\Psi_{x}}
$$

and

$$
\Psi_{z} d z+\Psi_{x} d x=0 .
$$

This means that $d \Psi=0$ and $\Psi=$ constant, so the streamfunction is constant along a streamline. In the next chapter we will use the streamfunction to simplify the governing equations for our problem. We also make use of vorticity which is defined as

$$
\zeta=\nabla \times \mathbf{u}
$$

In 3-dimensional space for a velocity vector $\mathbf{u}=(u, v, w)$, we can write the components of vorticity as

$$
\begin{aligned}
& \zeta_{x}=w_{y}-v_{z}, \\
& \zeta_{y}=u_{z}-w_{x}, \\
& \zeta_{z}=v_{x}-u_{y} .
\end{aligned}
$$

The physical essence of the vorticity is that it is equal to two times the local angular rate of rotation. In 2-dimensional space $(x, z)$, the vorticity has only a $y$-component $\zeta_{y}$ which we shall simply denote as $\zeta$. It is related to the streamfunction by

$$
\zeta=u_{z}-w_{x}=\Psi_{z z}+\Psi_{x x}=\nabla^{2} \Psi .
$$

We will use the streamfunction and vorticity to derive governing equations in the next chapter. 


\subsection{Some important parameters}

In our discussion of fluids so far, we have been assuming that all the fluid properties (dependent and independent variables) are measured in terms of pre-defined units, e.g. $\mathrm{m}$, sec, $\mathrm{K}$ etc., i.e. the equations are dimensional and all the terms in a given equation must have the same dimension.

In mathematical studies of fluids and other physical systems we often rewrite the governing equations in terms of nondimensional quantities by scaling all the variables and parameters in the equations with respect to some fixed reference values.

$$
\text { nondimensional quantity }=\frac{\text { dimensional quantity }}{\text { reference value of that quantity }} \text {. }
$$

The advantage of doing this is that in terms of nondimensional quantities it is easy to compare different problems that may have different physical scales. Two problems may be "dynamically similar" when written in terms of nondimensional quantities although they may have different scales in physical space. This allows us for example to predict the solution of one problem based on the solution of a dynamically similar problem, or to use experimental data from one problem to understand another dynamically similar one.

In section 3.1 we shall describe the process of nondimensionalization of the governing equations in our problem. In general, after nondimensionalization, certain nondimensional parameters arise. In this section we define and describe some of the important nondimensional parameters that are frequently used in the study of geophysical fluid flows. 


\section{Richardson number}

The Richardson number is named after Lewis Fry Richardson (1881 - 1953). It is a dimensionless number that expresses the ratio of potential to kinetic energy

$$
R i=\frac{g h}{u^{2}}
$$

where $g$ is the acceleration due to gravity, $h$ is a representative vertical lengthscale, and $u$ is a representative speed.

If the Richardson number is much less than unity, buoyancy is unimportant in the flow. If it is much greater than unity, buoyancy is dominant (in the sense that there is insufficient kinetic energy to homogenize the fluids). If the Richardson number is of order unity, then the flow is likely to be buoyancy-driven: the energy of the flow derives from the potential energy in the system originally.

In thermal convection problems, the Richardson number represents the importance of natural convection relative to the forced convection. The Richardson number in this context is defined as

$$
R i=\frac{g \beta \Delta T L}{U^{2}}
$$

where $\beta$ is the thermal expansion coefficient, $\Delta T$ is the change of temperature in the layer, $L$ is the characteristic length, and $U$ is the characteristic velocity.

Typically, natural convection is negligible when $R i \leq 0.1$, forced convection is negligible when $R i>10$, and neither is negligible when $0.1<R i<10$. It may be noted that usually the forced convection is large relative to natural convection except in the case of extremely low forced flow velocities.

In oceanography, the Richardson number has a more general form which takes stratification into account. It is a measure of the relative importance of inertial and 
density effects in the water column. It is defined as

$$
R i=\frac{N^{2}}{(d u / d z)^{2}}
$$

where $N$ is the frequency of free oscillations excited in the fluid because of unstable density stratification.

$$
N^{2}=-\frac{g}{\rho} \frac{d \rho}{d z}
$$

$N$ is called Brunt-Väisälä frequency.

The Richardson number defined above is always positive. An imaginary $N$ indicates unstable density gradients with active convective overturning. Under such circumstances, $N$ does not have an accepted quantitative meaning and the magnitude of negative $R i$ is not generally of interest. When $R i$ is small (typically considered below $1 / 4)$, then velocity shear is considered sufficient to overcome the tendency of a stratified fluid to remain stratified, and some mixing will generally occur. When $R i$ is large, turbulent mixing across the stratification is generally suppressed. It has been shown rigorously that $R i<1 / 4$ corresponds to instability. This is known as the Miles-Howard theorem and is described in section 3.4.

\section{Froude number}

Another important dimensionless parameter in the study of stratified flows is the Froude number Fr. The Froude number is named after William Froude $(1810-1879)$ who made numerous experiments on modelling the wave and frictional drag of ships. The nondimensional Froude number is defined as

$$
F r=U / c
$$


where $U$ is an average velocity, and $c$ is the wave propagation velocity. When the Froude number is considered in the context of the gravity waves it is defined as $F=$

$U / \sqrt{g l}$, where $\sqrt{g l}$ is the speed of gravity waves. So, the Froude number represents the fluid speed divided by the significant wave speed. The Froude number is analogous to the Mach number in compressible flows, defined as the ratio of the speed of flow to the speed of sound in the medium. The flow may be said to be subcritical if $F r<1$, critical if $F r=1$, and supercritical if $F r>1$.

\section{Reynolds number}

The Reynolds number is named after Osborne Reynolds (1842-1912), who proposed it in 1883. It is a measure of the ratio of inertial forces $(U / L)$ to viscous forces $\left(\nu / L^{2}\right)$ and, consequently, it quantifies the relative importance of these two types of forces for given flow conditions

$$
R e=U L / \nu
$$

The Reynolds number is one of the most important dimensionless number in fluid dynamics and is used to provide a criterion for determining dynamic similitude.

It is also used to identify and predict different flow regimes, such as laminar or turbulent flow. Laminar flow occurs at low Reynolds numbers, where viscous forces are dominant, and is characterized by smooth, constant fluid motion, while turbulent flow occurs at high Reynolds numbers and is dominated by inertial forces, which tend to produce random eddies, small-scales vortices and other flow fluctuations.

\section{Prandtl number}

The Prandtl number is a dimensionless number that approximates the ratio of momentum diffusivity (kinematic viscosity) and thermal diffusivity. It is named after 
the German physicist Ludwig Prandtl (1875 - 1953) and is defined as

$$
\operatorname{Pr}=\nu / K
$$

where $\nu$ is the kinematic viscosity, and $K$ is the thermal diffusivity, $K=\frac{\kappa}{\rho C_{p}}$ where $\kappa$ is the thermal conductivity and $C_{p}$ is the specific heat capacity. The Prandtl number compares the heat conduction with convection in the medium. For air this number is approximately $\operatorname{Pr} \approx 0.72$, for water $\operatorname{Pr} \approx 7$. It means that in air both convection and a heat conduction are significant processes, while in water convection provides a dominant input into the processes of heat transfer. 


\section{Chapter 3}

\section{Gravity waves in the atmosphere}

\subsection{Gravity waves: governing equations}

In this chapter the governing equations for gravity wave propagation will be derived.

The governing equations for this study are the equations of motion for a twodimensional fluid defined in terms of Cartesian coordinates $x$ and $z$ in the horizontal and vertical directions, respectively.

In the atmosphere density varies slowly in the vertical direction. So, we can use the Boussinesq approximation for our problem, and in the momentum equation we retain the density variation $\bar{\rho}(z)$ only in the gravitational term and use the constant reference value of density $\rho_{1}$ in all other terms as described in (Landau, Lifshitz, 1953). The momentum equation under the Boussinesq approximation (2.55) is:

$$
\frac{\partial \mathbf{u}}{\partial t}+(\mathbf{u} \cdot \nabla) \mathbf{u}=-\frac{\nabla p^{\prime}}{\rho_{1}}+\frac{\rho^{\prime}}{\bar{\rho}} \mathbf{g}+\nu \nabla^{2} \mathbf{u}
$$

where $\bar{\rho}$ is a function of $z: \bar{\rho}=\rho_{1} e^{-z / H}$, where $H$ is the height scale of the atmosphere.

To simplify the further consideration of the problem let us write the momentum 
equation in nondimensional form.

Let us denote all dimensional variables by an asterisk $*$ and write the equations as:

$$
\frac{\partial \mathbf{u}^{*}}{\partial t^{*}}+\left(\mathbf{u}^{*} \nabla\right) \mathbf{u}^{*}=-\frac{\nabla p^{\prime *}}{\rho_{1}^{*}}+\frac{\rho^{\prime *}}{\bar{\rho}^{*}} \mathbf{g} *+\nu \nabla^{2} \mathbf{u}^{*}
$$

with $\nu$ being the dimensional coefficient of kinematic viscosity. Let the characteristic length of our problem be $L$, the characteristic velocity be $U$ and the characteristic density be $\Re$. Then the characteristic time is $T=L / U$. We define the nondimensional variables in terms of the corresponding dimensional ones as follows:

$$
x=\frac{x^{*}}{L}, z=\frac{z^{*}}{L}, t=\frac{t^{*}}{T}=\frac{U t^{*}}{L}, \rho=\frac{\rho^{*}}{\Re}, p=\frac{p^{*}-p_{\infty}}{\Re U^{2}}, g=\frac{g^{*} L}{U^{2}} .
$$

This means that the derivatives in terms of non-dimensional variables will be:

$$
\begin{aligned}
\frac{\partial}{\partial x^{*}} & =\frac{\partial}{\partial x} \cdot \frac{\partial x}{\partial x^{*}}=\frac{1}{L} \cdot \frac{\partial}{\partial x} ; \\
\frac{\partial}{\partial z^{*}} & =\frac{1}{L} \frac{\partial}{\partial z} \\
\frac{\partial}{\partial t^{*}} & =\frac{1}{T} \frac{\partial}{\partial t} .
\end{aligned}
$$

So, $\nabla^{*}=(1 / L) \nabla$. We write the equation (3.2) in terms of non-dimensional variables as

$$
\frac{U^{2}}{L} \frac{\partial \mathbf{u}}{\partial t}+\frac{U^{2}}{L}(\mathbf{u} \cdot \nabla) \mathbf{u}=-\frac{1}{L} \Re U^{2} \frac{\nabla p^{\prime}}{\Re \rho_{1}}+\frac{U^{2}}{L} \frac{\rho^{\prime}}{\bar{\rho}} \mathbf{g}+\nu \frac{U}{L^{2}} \nabla^{2} \mathbf{u}
$$

After some simplifications we get

$$
\frac{\partial \mathbf{u}}{\partial t}+(\mathbf{u} \cdot \nabla) \mathbf{u}=-\frac{\nabla p^{\prime}}{\rho_{1}}+\frac{\rho^{\prime}}{\bar{\rho}} \mathbf{g}+\frac{\nu}{L U} \nabla^{2} \mathbf{u}
$$

We define the nondimensional parameter Reynolds number $R e=U L / \nu$. The physical essence of the parameter was discussed in section 2.5. In terms of the Reynolds number 
the nondimensional momentum equation can be rewritten as

$$
\frac{\partial \mathbf{u}}{\partial t}+(\mathbf{u} \nabla) \mathbf{u}=-\frac{\nabla p^{\prime}}{\rho_{1}}+\frac{\rho^{\prime}}{\bar{\rho}} \mathbf{g}+R e^{-1} \nabla^{2} \mathbf{u}
$$

The Reynolds number describes the relation between inertial and viscous forces. For geophysical flows with large scales and relatively small viscosity the Reynolds number is usually very large, $R e^{-1}<<1$, and viscous terms in the momentum equation can be neglected as far as we ignore microscopic fluid scales. We shall make this approximation in deriving our analytic solutions. The numerical solutions however include a viscous term as described in chapter 7 .

Under the Boussinesq approximation the dimensionless continuity equation is

$$
u_{x}+w_{z}=0
$$

where $u$ and $w$ are the horizontal and vertical components of the velocity and the subscripts denote partial differentiation. This allows us to define a streamfunction by

$$
u=-\Psi_{z}, \quad w=\Psi_{x}
$$

so that the horizontal and vertical momentum equations can be combined into a single equation for the streamfunction (see section 2.4). The function $\Psi(x, z, t)$ is the total streamfunction; it is decomposed into a contribution from the steady basic flow and a time dependent perturbation in the form of a gravity wave packet:

$$
\Psi(x, z, t)=\bar{\psi}(z)+\varepsilon \psi(x, z, t)
$$

It is assumed that $\psi \sim O(1)$, so the parameter $\varepsilon$ gives a measure of the magnitude of 
the waves relative to that of the basic flow. If the gravity waves are assumed to be forced by a mountain then $\varepsilon$ is determined by the height of the mountain.

The basic flow is a shear flow depending on $z$ and independent of $x$; it has a horizontal component only, so the basic flow has velocity $(\bar{u}, 0)$, where

$$
\bar{u}(z)=-\bar{\psi}_{z}
$$

Thus, the total horizontal velocity of the fluid flow is

$$
u_{\mathrm{total}}=\bar{u}(z)+\varepsilon u(x, z, t)
$$

and the vertical velocity is

$$
w_{\text {total }}=\varepsilon w(x, z, t)
$$

since $\bar{w}=\bar{\psi}_{x}=0$. The other dependent variables are also separated into mean and perturbation parts, for example, the total density is written as $\rho_{\text {total }}(x, z, t)=$ $\bar{\rho}(z)+\varepsilon \rho(x, z, t)$. From this point on we will work with equations written in terms of the perturbation variables. So we will represent the perturbations by $\psi, u, w, p, \rho$ and so on without primes.

\section{Vorticity equation}

We shall rewrite our equations in streamfunction-vorticity formulation. The definition and physical interpretation of the streamfunction and vorticity was given in section 2.4. According to (2.83) the perturbation of vorticity $\zeta$ is defined as

$$
\zeta=\nabla^{2} \psi
$$


We can derive an equation for the vorticity using the momentum equation. The horizontal and vertical Navier-Stokes equations are respectively:

$$
\begin{gathered}
\frac{\partial u}{\partial t}+\bar{u} \frac{\partial u}{\partial x}+\varepsilon u \frac{\partial u}{\partial x}+w \frac{\partial \bar{u}}{\partial z}+\varepsilon w \frac{\partial u}{\partial z}=-\frac{1}{\rho_{1}} \frac{\partial p}{\partial x}+R e^{-1} \nabla^{2} u+\varepsilon^{-1} R e^{-1} \bar{u}^{\prime \prime}, \\
\frac{\partial w}{\partial t}+\bar{u} \frac{\partial w}{\partial x}+\varepsilon u \frac{\partial w}{\partial x}+\varepsilon w \frac{\partial w}{\partial z}=-\frac{1}{\rho_{1}} \frac{\partial p}{\partial z}-\frac{\rho}{\bar{\rho}} g+R e^{-1} \nabla^{2} w
\end{gathered}
$$

In terms of the streamfunction $\psi$ these equations can be written as

$$
-\left(\frac{\partial}{\partial t}+\bar{u} \frac{\partial}{\partial x}\right) \psi_{z}+\varepsilon\left(\psi_{z} \psi_{x z}-\psi_{x} \psi_{z z}\right)+\bar{u}_{z}^{\prime} \psi_{x}=-\frac{1}{\rho_{1}} \frac{\partial p}{\partial x}-R e^{-1} \nabla^{2} \psi_{z}-\varepsilon^{-1} R e^{-1} \bar{u}^{\prime \prime}
$$

$$
\left(\frac{\partial}{\partial t}+\bar{u} \frac{\partial}{\partial x}\right) \psi_{x}-\varepsilon\left(\psi_{z} \psi_{x x}+\psi_{x} \psi_{x z}\right)=-\frac{1}{\rho_{1}} \frac{\partial p}{\partial z}-\frac{\rho}{\bar{\rho}} g+R e^{-1} \nabla^{2} \psi_{x}
$$

Subtracting the $x$-derivative of the $z$ momentum equation from the $z$-derivative of the $x$ momentum equation we get an equation for the perturbation vorticity $\zeta$ and perturbation streamfunction $\psi$ :

$$
\zeta_{t}+\bar{u} \zeta_{x}-\bar{u}^{\prime \prime} \psi_{x}+g(\bar{\rho})^{-1} \rho_{x}+\varepsilon\left(\psi_{x} \zeta_{z}-\psi_{z} \zeta_{x}\right)-R e^{-1} \nabla^{2} \zeta+R e^{-1} \varepsilon^{-1} \bar{u}^{\prime \prime \prime}=0
$$

In the two equations (3.16) and (3.21) we have three dependent variables $\psi, \zeta$ and $\rho$. So we need a third equation for $\rho$ to complete the system. Under the Boussinesq approximation an equation for the density can be derived from the heat equation (2.67), as was shown in section 2.3. According to (2.73)

$$
\left(\frac{\partial}{\partial t}+\mathbf{u} \cdot \nabla\right) \rho_{\text {total }}=K \nabla^{2} \rho_{\text {total }}
$$


Taking into account that $\rho_{\text {total }}=\bar{\rho}+\varepsilon \rho$ we can rewrite equation (3.22) in terms of the perturbations $\rho, u, w$;

$$
\rho_{t}+\bar{u} \rho_{x}+\varepsilon w \rho_{z}+\varepsilon u \rho_{x}=\varepsilon^{-1} K \bar{\rho}_{z z}+K \nabla^{2} \rho
$$

or, in terms of the streamfunction $\psi$ as

$$
\rho_{t}+\bar{u} \rho_{x}+\bar{\rho}^{\prime} \psi_{x}+\varepsilon\left(\psi_{x} \rho_{z}-\psi_{z} \rho_{x}\right)-K \nabla^{2} \rho-K \varepsilon^{-1} \bar{\rho}^{\prime \prime}=0
$$

If the equation is written in nondimensional form

$$
\rho_{t}+\bar{u} \rho_{x}+\bar{\rho}^{\prime} \psi_{x}+\varepsilon\left(\psi_{x} \rho_{z}-\psi_{z} \rho_{x}\right)-\frac{K}{U L} \nabla^{2} \rho-\frac{K}{U L} \varepsilon^{-1} \bar{\rho}^{\prime \prime}=0
$$

there is a nondimensional combination $K / U L$ in the last two terms of the equation. We can represent this combination in terms of the Reynolds number $(2.90)$ and the Prandtl number (2.91):

$$
\frac{K}{U L}=\frac{\nu}{U L} \frac{K}{\nu}=\operatorname{Re}^{-1} \operatorname{Pr}^{-1}
$$

and we rewrite the equation as

$$
\rho_{t}+\bar{u} \rho_{x}+\bar{\rho}^{\prime} \psi_{x}+\varepsilon\left(\psi_{x} \rho_{z}-\psi_{z} \rho_{x}\right)-\operatorname{Re}^{-1} \operatorname{Pr}^{-1} \nabla^{2} \rho-\operatorname{Re}^{-1} \operatorname{Pr}^{-1} \varepsilon^{-1} \bar{\rho}^{\prime \prime}=0
$$

Now we have three equations, (3.16), (3.21) and (3.27), with three dependent variables $\psi, \rho$ and $\zeta$.

The Prandtl number for air is $\approx 0.72$. It is assumed that $R e>>1$ which is an appropriate assumption for geophysical flows with large scales and relatively small viscosity. Thus, the last terms in equations (3.21) and (3.27) can be neglected and we 
shall do so in our analytical solutions. In the numerical solution described in Chapter 7 these terms are included because, although they are small, their presence reduces numerical instabilities.

\subsection{The Taylor-Goldstein equation}

To describe gravity wave propagation, instead of the three equations (3.16), (3.21) and (3.27), it is convenient to work with one equation with one dependent variable $\psi$. In the absence of viscosity and nonlinearity it is straightforward to combine these three equations into one. Setting to zero the viscous and non-linear terms in equation (3.21) and taking into account that $\zeta=\nabla^{2} \psi$, we get

$$
\left(\frac{\partial}{\partial t}+\bar{u} \frac{\partial}{\partial x}\right) \nabla^{2} \psi-\bar{u}^{\prime \prime}(z) \psi_{x}-g \frac{\rho_{x}}{\bar{\rho}(z)}=0
$$

Similarly, setting to zero the heat conduction and nonlinear terms in (3.27), we get

$$
\left(\frac{\partial}{\partial t}+\bar{u} \frac{\partial}{\partial x}\right) \rho+\psi_{x} \frac{\partial \rho}{\partial z}=0
$$

If we apply the linear differential operator $\left(\frac{\partial}{\partial t}+\bar{u} \frac{\partial}{\partial x}\right)$ to equation (3.28) and $\left(\frac{\partial}{\partial x}\right)$ to equation (3.29), we obtain

$$
\left(\frac{\partial}{\partial t}+\bar{u} \frac{\partial}{\partial x}\right)^{2} \nabla^{2} \psi-\bar{u}^{\prime \prime}(z)\left(\frac{\partial}{\partial t}+\bar{u} \frac{\partial}{\partial x}\right) \psi_{x}+\left(\frac{\partial}{\partial t}+\bar{u} \frac{\partial}{\partial x}\right) g \frac{\rho_{x}}{\bar{\rho}(z)}=0
$$

and

$$
\left(\frac{\partial}{\partial t}+\bar{u} \frac{\partial}{\partial x}\right) \rho_{x}+\psi_{x x} \frac{d \bar{\rho}(z)}{d z}=0
$$


Combining these two equations we get an equation for $\psi$ :

$$
\left(\frac{\partial}{\partial t}+\bar{u} \frac{\partial}{\partial x}\right)^{2} \nabla^{2} \psi-\bar{u}^{\prime \prime}(z)\left(\frac{\partial}{\partial t}+\bar{u} \frac{\partial}{\partial x}\right) \psi_{x}-\frac{g}{\bar{\rho}} \frac{d \bar{\rho}(z)}{d z} \psi_{x x}=0
$$

The last term in this equation represents the density change due to the vertical density stratification. As described in section 2.5 we can define the buoyancy frequency, or Brunt-Väisälä frequency by

$$
N^{2}=-\frac{g}{\bar{\rho}} \frac{d \bar{\rho}}{d z}
$$

A good approximation to the background density profile of the atmosphere is the exponential function $\bar{\rho}(z)=\rho_{1} e^{-z / H}$. With this density profile $N^{2}=g / H$ and $N$ is constant.

Finally, we have a single equation of streamfunction perturbation $\psi$

$$
\left(\frac{\partial}{\partial t}+\bar{u} \frac{\partial}{\partial x}\right)^{2} \nabla^{2} \psi-\bar{u}^{\prime \prime}(z)\left(\frac{\partial}{\partial t}+\bar{u} \frac{\partial}{\partial x}\right) \psi_{x}+N^{2} \psi_{x x}=0
$$

We now show how this equation can be used to set an equation for the wave amplitude.

\subsection{Normal mode solution and the Taylor-Goldstein equation}

Gravity waves in the atmosphere and ocean are in general approximately periodic in time and in the horizontal direction. Based on that observation we can assume the solutions of (3.34) are of the form

$$
\psi(x, z, t)=\phi(z) e^{i k(x-c t)}+\text { c.c. }
$$


where c.c. defines the complex conjugate of the preceding term, $\phi$ is the complex wave amplitude, $k$ is the horizontal component of a wave number, $c$ is the horizontal phase speed of wave propagation, $k$ and $c$ are real numbers, $k$ is positive. The horizontal wavelength is $2 \pi / k$ and the wave period is $2 \pi / k c$. The expression (3.35) is called a normal mode solution. By adding the complex conjugate we ensure that $\psi$ is real although $\phi$ generally has a nonzero imaginary part. After substituting this normal mode solution into equation (3.34) we get an ordinary differential equation for $\phi(z)$ :

$$
(\bar{u}-c)^{2}\left(\phi_{z z}-k^{2} \phi\right)-(\bar{u}-c) \bar{u}_{z z} \phi+N^{2} \phi=0 .
$$

In our problem, in which we consider the gravity waves forced by topography the horizontal length scale of the problem $L_{x}$ is much larger then the vertical scale $L_{z}$. So, it makes sense to insert in our consideration a small parameter $\delta$ which is defined as the square of the aspect ratio: $\delta=\left(L_{z} / L_{x}\right)^{2}$. We will define a new variable $z=z_{\text {old }}\left(L_{x} / L_{z}\right)$. In terms of the new variables our equation (3.36) becomes

$$
(\bar{u}-c)^{2}\left(\frac{\partial^{2}}{\partial z^{2}}-\delta k^{2}\right) \phi-(\bar{u}-c) \bar{u}_{z z} \phi+N^{2} \phi=0
$$

This is the Taylor-Goldstein equation (Taylor, 1931), (Goldstein, 1931) and it governs the behaviour of wave perturbations in a stratified parallel flow. We now describe solutions for the Taylor-Goldstein equation.

\section{The solution of the Taylor-Goldstein equation for the case $\bar{u}=$ constant}

We begin our examination of the solutions of the Taylor-Goldstein equation (3.37) with the simple case of constant background stratification $N$ and constant background wind 
$\bar{u}$ and use this simple example to illustrate how to find the group velocity and direction of gravity wave propagation. Under these assumptions equation (3.37) simplifies to

$$
\frac{d^{2} \phi}{d z^{2}}+\left(\frac{N^{2}}{(\bar{u}-c)^{2}}-\delta k^{2}\right) \phi=0
$$

where $k$ is the horizontal component of the wave vector, and $c$ is a horizontal component of the phase speed. We define the wave frequency $\omega=c k$. To describe wave propagation relative to the basic flow we also define the intrinsic frequency

$$
\Omega=\omega-\bar{u} k
$$

Following Booker and Bretherton (1967) we look for the general solution of the TaylorGoldstein equation (3.38) in the form

$$
\phi(z)=A e^{i m z}+B e^{-i m z}
$$

where $A$ and $B$ are arbitrary constants, and the vertical wavenumber $m$ is given by

$$
m^{2}=\frac{N^{2}}{(\bar{u}-c)^{2}}-\delta k^{2}
$$

Define $k$ positive. If $\frac{N^{2}}{(\bar{u}-c)^{2}}<\delta k^{2}$ then $m$ is complex and we have solutions that either grow or decay with height. However we only consider cases where $\delta \ll 1$ and $\frac{N^{2}}{(\bar{u}-c)^{2}}>>k^{2}$ which is the situation that occurs in real geophysical flows. Thus $m$ is real and the solutions are sinusoidal in the vertical direction and their amplitude is constant. We define $m$ such that $m \sim \frac{N}{(\bar{u}-c)}$, so that $\operatorname{sign}[m]=\operatorname{sign}\left[\frac{N}{(\bar{u}-c)}\right]$. Following Booker and Bretherton (1967) we can show that the solution $e^{i m z}$ corresponds to an upward-propagating wave, with positive vertical group velocity $c_{g r}>0$, and the 
solution $e^{-i m z}$ corresponds to a downward-propagating wave with negative vertical group velocity $c_{g r}<0$. The frequency is

$$
\omega=k \bar{u} \pm \frac{k N}{\left(\delta k^{2}+m^{2}\right)^{1 / 2}}
$$

The horizontal component of the wave phase speed is $c=\omega / k=\bar{u} \pm \frac{N}{\left(\delta k^{2}+m^{2}\right)^{1 / 2}}$. For the solution with the plus sign, $e^{i m z}$, the vertical component of the group velocity is

$$
c_{g r}=\frac{\partial \omega}{\partial m}=\mp \frac{k N m}{\left(\delta k^{2}+m^{2}\right)^{3 / 2}}
$$

Using the fact that

$$
\mp \frac{N}{\left(\delta k^{2}+m^{2}\right)^{1 / 2}}=\bar{u}-c \Rightarrow \mp \frac{N^{3}}{\left(\delta k^{2}+m^{2}\right)^{3 / 2}}=(\bar{u}-c)^{3} \text {, }
$$

(3.43) can be rewritten as

$$
c_{g r}=\frac{k m(\bar{u}-c)^{3}}{N^{2}}
$$

Since by definition $m$ and $\bar{u}-c$ are of the same sign, $(\bar{u}-c)^{3}$ is always positive and $c_{g r}$ is positive, regardless of the sign of $m$ and $\bar{u}-c$. This means the solution $e^{i m z}$ corresponds to an upward-propagating wave.

Similarly for the solution $e^{-i m z}$ which has vertical wavenumber $-m$ the group velocity is

$$
c_{g r}=\frac{\partial \omega}{\partial(-m)}=-\frac{\partial \omega}{\partial m}= \pm \frac{k N m}{\left(\delta k^{2}+m^{2}\right)^{3 / 2}}=-\frac{k m(\bar{u}-c)^{3}}{N^{2}}
$$

As before $m(\bar{u}-c)^{3}$ is always positive and so $c_{g r}$ is negative. This means the solution $e^{-i m z}$ corresponds to a downward-propagating wave.

Throughout this thesis we shall only consider solutions that correspond to upward- 
propagating waves. This is because our waves are forced at the lower boundary of the domain under consideration (by a mountain or other obstacle at ground level) and propagate upwards. It is assumed there is no incoming wave energy at infinity.

Note that the solution corresponding to a downward-propagating wave would be relevant only in a situation where the waves are assumed to be forced be a high-altitude source, e.g. by radiation in the ionosphere, but in the present context we are interested in upward-propagating topographic waves in the lower atmosphere (troposphere).

To find vectors of the group velocity and phase velocity let us consider the dispersion relation in terms of the intrinsic frequency $\Omega$ :

$$
\Omega= \pm \frac{k N}{\left(\delta k^{2}+m^{2}\right)^{1 / 2}}
$$

From the dispersion relation we can find the group velocity relative to the basic flow. For wave of the form $e^{i m z}$ the horizontal component of the group velocity is

$$
c_{g r x}=\frac{\partial \Omega}{\partial k}=\frac{N m^{2}}{\left(\delta k^{2}+m^{2}\right)^{3 / 2}},
$$

and the vertical component as we have already shown is

$$
c_{g r z}=\frac{\partial \Omega}{\partial m}=-\frac{N m k}{\left(\delta k^{2}+m^{2}\right)^{3 / 2}} .
$$

So, the vector of the group velocity is

$$
\mathbf{c}_{g r}=\frac{N m}{\left(\delta k^{2}+m^{2}\right)^{3 / 2}} \quad(m,-k) .
$$


The relative phase velocity of the perturbation has horizontal and vertical components:

$$
\begin{gathered}
c_{x}=\frac{\Omega}{k}=\frac{N}{\left(\delta k^{2}+m^{2}\right)^{1 / 2}}, \\
c_{z}=\frac{\Omega}{m}=\frac{N k}{\left(\delta k^{2}+m^{2}\right)^{1 / 2} m} .
\end{gathered}
$$

So, the phase velocity is

$$
\mathbf{c}=\frac{N m}{k\left(\delta k^{2}+m^{2}\right)^{3 / 2}} \quad(k, m)
$$

The scalar product of the group velocity and the phase velocity of a wave equals zero, $\mathbf{c} \cdot \mathbf{c}_{g r}=0$, and we can conclude that vectors $\mathbf{c}$ and $\mathbf{c}_{g r}$ are perpendicular and have opposite vertical components (see Figure 3.1).

The spatial distribution of the velocity associated with the first term $e^{i m z}$ in the solution $(3.40)$ is

$$
\begin{gathered}
w=w_{0} \exp (i(k x+m z-\omega t)), \\
u=u_{0} \exp (i(k x+m z-\omega t)) .
\end{gathered}
$$

It represents a plane wave with the phase front

$$
k x+m z-\omega t=\text { constant } .
$$

Substituting (3.54) and (3.55) into the continuity equation we get $u_{x}+w_{z}=0$ and $k u+m w=0$. This means that the velocity vector and the wave number vector are perpendicular as $(k, m) \cdot(u, w)=0$, and we can conclude that particles in the gravity wave move perpendicular to the direction of wave propagation. This is a characteristic 


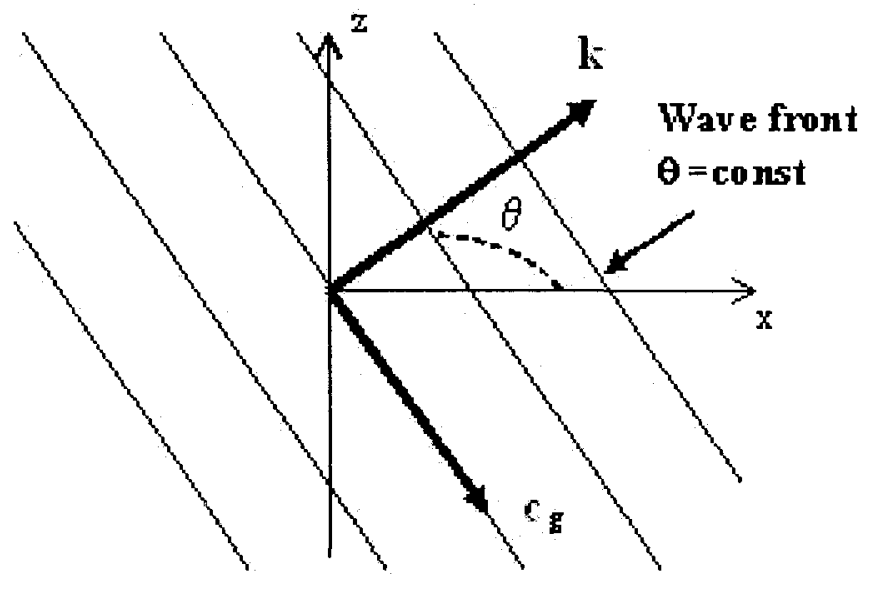

Figure 3.1: Propagation of gravity waves. The gravity waves propagate with the phase speed $c$. The wave number vector $\mathbf{k}(k, m)$ points in the direction of the wave propagation. Lines of constant phase (wave front) are given by $\theta=k x+m z-\omega t=$ constant. The vector of the group velocity $\mathbf{c}_{\mathbf{g}}$ is perpendicular to the wavenumber vector $\mathbf{k}$. The vectors $\mathbf{k}$ and $\mathbf{c}_{\mathbf{g}}$ have vertical components of opposite signs. 
feature of internal gravity waves.

\subsection{Stability of stratified shear flows}

In this section we derive a well-known criterion for the stability of stratified shear flows which is very important for our future consideration. This criterion was first derived by Miles (1961) and Howard (1961).

First we define what it means to say that a flow is stable or unstable. Assume a perturbation in the flow of the form $e^{i k(x-c t)}$, where $c$ is complex, $c=c_{R}+i c_{I}$. So the perturbation can be written as $e^{i k\left(x-c_{R} t\right)} e^{k c_{I} t}$. If the imaginary part of $c$ is nonzero then the amplitude of the perturbation changes with time. If $c_{I}<0$ the amplitude decays with time and the flow is stable. If $c_{I}>0$ the amplitude increases with time and the flow is unstable. If $c_{I}=0$ the flow is neutrally stable.

'To derive a stability criterion we assume a two-dimensional, frictionless, irrotational flow, make the Boussinesq approximation and consider the Taylor-Goldstein equation (3.37). The stability criterion is defined for flow in a layer of finite depth, say $0 \leq z \leq H$, with zero boundary conditions at the top and bottom $\phi(0)=\phi(H)=0$. To obtain a necessary condition for linear instability we define a new variable $\tilde{\phi}$ by

$$
\tilde{\phi}(z)=\phi(z)(\bar{u}(z)-c)^{-1 / 2}
$$

The derivatives of $\phi$ can then be expressed in terms of $\tilde{\phi}$ :

$$
\begin{gathered}
\phi_{z}=(\bar{u}-c)^{1 / 2} \tilde{\phi}_{z}+\frac{1}{2} \tilde{\phi}_{\bar{u}}(\bar{u}-c)^{-1 / 2} \\
\phi_{z z}=(\bar{u}-c)^{1 / 2} \tilde{\phi}_{z z}+\frac{\bar{u}_{z} \tilde{\phi}_{z}+\frac{1}{2} \tilde{\phi} \bar{u}_{z z}}{(\bar{u}-c)^{1 / 2}}-\frac{1}{4} \frac{\tilde{\phi} \bar{u}_{z}^{2}}{(\bar{u}-c)^{3 / 2}} .
\end{gathered}
$$


The Taylor-Goldstein equation then becomes, after some simplifications,

$$
\frac{d}{d z}(\bar{u}-c) \tilde{\phi}_{z}=\delta k^{2}(\bar{u}-c) \tilde{\phi}+\frac{1}{2} \bar{u}_{z z} \tilde{\phi}+\frac{(1 / 4) \bar{u}_{z}^{2}-N^{2}}{\bar{u}-c} \tilde{\phi}
$$

Now we multiply the last equation by $\tilde{\phi}^{*}$ (the complex conjugate of $\tilde{\phi}$ ) and integrate it from $z=0$ to $z=H$, where $H$ is the depth of the layer. The boundary conditions are $w=0$ on the rigid boundaries at $z=0$ and $z=H$. This is possible if

$$
\phi(0)=\phi(H)=0
$$

and

$$
\tilde{\phi}(0)=\tilde{\phi}(H)=0 \text {. }
$$

The first term in (3.60) gives

$$
\begin{array}{r}
\int_{0}^{H} \frac{d}{d z}\left[(\bar{u}-c) \tilde{\phi}_{z}\right] \tilde{\phi}^{*} d z=\int_{0}^{H}\left[\frac{d}{d z}(\bar{u}-c) \tilde{\phi}_{z} \tilde{\phi}^{*}\right]-(\bar{u}-c) \tilde{\phi}_{z} \tilde{\phi}^{*} d z= \\
-\int_{0}^{H}(\bar{u}-c)\left|\tilde{\phi}_{z}\right|^{2} d z
\end{array}
$$

where we have used $\tilde{\phi}=0$ at the boundaries. After some manipulation we finally obtain

$$
\int_{0}^{d} \frac{N^{2}-(1 / 4) \bar{u}_{z}^{2}}{\bar{u}-c}|\tilde{\phi}|^{2} d z=\int_{0}^{d}(\bar{u}-c)\left(\left|\tilde{\phi}_{z}\right|^{2}+\delta k^{2}|\tilde{\phi}|^{2}\right) d z+\frac{1}{2} \int_{0}^{d} \bar{u}_{z z}|\tilde{\phi}|^{2} d z
$$

As before, let $c=c_{R}+i c_{I}$ The imaginary part of equation (3.64) gives

$$
c_{I} \int_{0}^{H} \frac{N^{2}-(1 / 4) \bar{u}_{z}^{2}}{|\bar{u}-c|^{2}}|\tilde{\phi}|^{2} d z=-c_{I} \int_{0}^{H}\left(\left|\tilde{\phi}_{z}\right|^{2}+\delta k^{2}|\tilde{\phi}|^{2}\right) d z
$$


The integral on the right-hand side of equation (3.65) is positive. So, if $N^{2}>(1 / 4) \bar{u}_{z}^{2}$ then $c_{I}=0$, and we can guarantee the stability. Usually this criterion is written in terms of the Richardson number

$$
\operatorname{Ri}(z)=\frac{N^{2}}{\bar{u}_{z}^{2}}
$$

and linear stability is guaranteed if the inequality

$$
R i>\frac{1}{4}
$$

is satisfied everywhere in the flow. So, we can formulate the Miles-Howard theorem (Miles (1961), Howard (1961)): A stratified shear flow is stable everywhere where $\mathbf{R i}>\mathbf{1} / 4$. This criterion does not state that the flow is necessarily unstable if $R i<\frac{1}{4}$ somewhere in the flow. This is a sufficient criterion for linear stability.

Note that this criterion applies strictly to steady linear perturbations that satisfy the Taylor-Goldstein equation. However, it is generally extended and applied in the context of linear time-dependent perturbations as well.

Throughout this thesis, in both the analytic and numerical solutions, we will only consider configurations where $R i>1 / 4$ everywhere in the flow at least initially. So at least initially the flow is stable. 


\section{Chapter 4}

\section{Exact solutions of the}

\section{Taylor-Goldstein equation}

In this chapter we will describe exact solutions of the Taylor-Goldstein equation (3.37) for some special cases. The Taylor-Goldstein equation has a singularity at the point where $\bar{u}(z)=c$. In section 4.1 we will use the method of Frobenius to solve the equation for the general case where $\bar{u}$ is an arbitrary function of $z$. In section 4.2 we will describe the solution of the Taylor-Goldstein equation for the case where $\bar{u}$ is a linear function of $z$ and the aspect ratio $\delta \neq 0$; the solution will be expressed in terms of the modified Bessel function. In section 4.3 we describe the case where $\bar{u}$ is a linear function of $z$ and $\delta=0$. We also discuss the critical-level behaviour for this solution. 


\subsection{Solution of the Taylor-Goldstein equation: the general case, using the method of Frobenius}

In Chapter 3 we derived a linearized equation describing the gravity wave propagation,

$$
\left(\frac{\partial}{\partial t}+\bar{u} \frac{\partial}{\partial x}\right)^{2}\left(\delta \frac{\partial^{2}}{\partial x^{2}}+\frac{\partial^{2}}{\partial z^{2}}\right) \psi-\bar{u}^{\prime \prime}\left(\frac{\partial}{\partial t}+\bar{u} \frac{\partial}{\partial x}\right) \psi_{x}+N^{2} \psi_{x x}=0
$$

where $\delta=L_{z}^{2} / L_{x}^{2}$. We assumed a solution to be in the form of a normal mode solution

$$
\psi(x, z, t)=\phi(z) e^{i k(x-c t)}+\text { c.c. }
$$

where c.c. defines the complex conjugate of the preceding term and obtained the Taylor-Goldstein equation for the amplitude $\phi(z)$ (3.37):

$$
(\bar{u}-c)^{2}\left(\frac{\partial^{2}}{\partial z^{2}}-\delta k^{2}\right) \phi-(\bar{u}-c) \bar{u}_{z z} \phi+N^{2} \phi=0
$$

This equation has a singularity at the singular point $z_{c}$ where $\bar{u}\left(z_{c}\right)=c$. In the atmosphere this level where the wave phase speed $c$ is equal to the background wind speed $\bar{u}$ is called the critical level. The region surrounding the singular level is called the critical layer. Let us find a general solution of the equation if $\bar{u}$ is a known function of $z$, analytic at the point $z=z_{c}$.

\section{Solution of the Taylor-Goldstein equation for an arbitrary function $\bar{u}(z)$}

Assuming that $\bar{u}$ is an analytic function in a neighbourhood of the singular point $z=z_{c}$, we can expand the function $\bar{u}(z)$ into a series in powers of $\left(z-z_{c}\right)$ : 


$$
\bar{u}=c+\left.\bar{u}^{\prime}\right|_{z_{c}}\left(z-z_{c}\right)+\left.\frac{1}{2} \bar{u}^{\prime \prime}\right|_{z_{c}}\left(z-z_{c}\right)^{2}+\ldots
$$

After substituting this series into (4.3) we can solve the equation using the Frobenius method

$$
\phi(z)=\sum_{n=0}^{\infty} a_{n}\left(z-z_{c}\right)^{n+\alpha} .
$$

We substitute the series (4.4) and (4.5) into the Taylor-Goldstein equation (4.3) and get:

$$
\begin{array}{r}
\sum_{n=0}^{\infty}(\alpha+n)(\alpha+n-1) a_{n}\left(z-z_{c}\right)^{\alpha+n-2}\left(\bar{u}^{\prime 2}\left(z-z_{c}\right)^{2}+\bar{u}^{\prime} \bar{u}^{\prime \prime}\left(z-z_{c}\right)\right)+ \\
N^{2} \sum_{n=0}^{\infty} a_{n}\left(z-z_{c}\right)^{\alpha+n}-\bar{u}^{\prime} \bar{u}^{\prime \prime} \sum_{n=0}^{\infty} a_{n}\left(z-z_{c}\right)^{\alpha+n+1}=0 .
\end{array}
$$

Comparing coefficients of the powers $\left(z-z_{c}\right)^{\alpha}$ we get a quadratic equation for $\alpha$ :

$$
a_{0}(\alpha-1) \alpha+a_{0} \frac{N^{2}}{\bar{u}_{c}^{\prime 2}}=0
$$

So,

$$
\alpha=\frac{1}{2} \pm i \sqrt{\frac{N^{2}}{\bar{u}_{c}^{\prime 2}}-\frac{1}{4}} .
$$

We consider only the upward propagation of gravity waves that corresponds to the plus sign

$$
\phi(z)=a_{0}\left(z-z_{c}\right)^{\frac{1}{2}+i \sqrt{\frac{N^{2}}{\bar{u}_{z}^{\prime 2}}-\frac{1}{4}}}
$$

Comparing coefficients of a power $\left(z-z_{c}\right)^{\alpha+1}$ we get an equation for $a_{1}$ :

$$
a_{1}\left[\left(\alpha^{2}+\alpha\right) \bar{u}^{\prime 2}+N^{2}\right]=\bar{u}^{\prime} \bar{u}^{\prime \prime} a_{0}\left(1-\alpha^{2}+\alpha\right)
$$


After some simplification we get an expression for $a_{1}$ :

$$
a_{1}=a_{0} \frac{\bar{u}^{\prime \prime}}{4 N^{2}}
$$

So, now we have the first two terms of the solution:

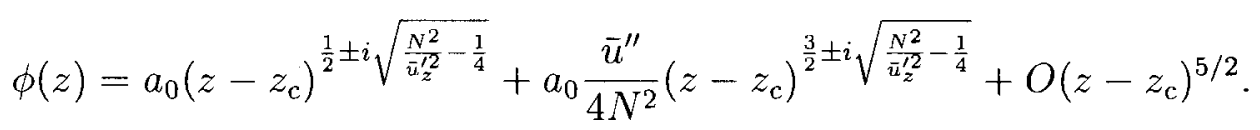

We define

$$
\gamma=\sqrt{\frac{N^{2}}{\bar{u}_{z}^{\prime 2}}-\frac{1}{4}}=\sqrt{R i-\frac{1}{4}}
$$

We are only considering the case where $R i>1 / 4$ and the flow is stable according to the Miles-Howard theorem. So, $\gamma$ is real. This series converges for $\left|z-z_{c}\right|<1$. So, we have got a solution of the Taylor-Goldstein equation (4.12) which is valid in a region close to a critical layer.

\subsection{Solution of the Taylor-Goldstein equation: $\bar{u}$ is a linear function of $z, \delta \neq 0$}

Let us consider the solution of the Taylor-Goldstein equation for the case where $\bar{u}$ is a linear function of $z: \bar{u}(z)-c=z-z_{c}$. In this case, (4.3) can be rewritten as

$$
\left(z-z_{c}\right)^{2}\left(-\delta k^{2}+\frac{\partial^{2}}{\partial z^{2}}\right) \phi-N^{2} \phi=0 .
$$


This equation can be written as a modified Bessel's equation by making a change of variables. Let us define $y=\left(z-z_{c}\right)$ and $\phi=\sqrt{y} g(y)$. Then the derivatives are

$$
\begin{array}{r}
\phi^{\prime}=\frac{g}{2 \sqrt{y}}+\sqrt{y} g^{\prime}, \\
\phi^{\prime \prime}=g^{\prime \prime} \sqrt{y}+\frac{g^{\prime}}{\sqrt{y}}-\frac{g}{4 y^{3 / 2}},
\end{array}
$$

where the primes denote differentiation with respect to the new variable $y$. In terms of the new variables $y$ and $g$ our equation takes the form

$$
y^{2} g^{\prime \prime}+y g^{\prime}+\left(-\delta k^{2} y^{2}-1 / 4+N^{2}\right) g=0 .
$$

To get the Bessel equation from (4.17) we have to make another change of variables:

$$
Y=i \sqrt{\delta} k\left(z-z_{c}\right)=i \sqrt{\delta} k y
$$

Let us note that we can do it only if $\delta \neq 0$. With the new variable, equation (4.17) takes the form of the modified Bessel equation

$$
Y^{2} g^{\prime \prime}+Y g^{\prime}+\left[Y^{2}-\left(i \sqrt{N^{2}-1 / 4}\right)^{2}\right] g=0
$$

The solutions of this equation are written in terms of the modified Bessel functions $J_{ \pm i \gamma}(i Y)$, or $J_{ \pm i \gamma}\left(i \sqrt{\delta} k\left(z-z_{c}\right)\right.$ ), where $\gamma=\sqrt{N^{2}-1 / 4}=\sqrt{R i-1 / 4}$ (note that $\bar{u}_{z}=1$, so $R i=N^{2}$ ). The general solution of (4.19) is

$$
g=A J_{i \gamma}(i Y)+B J_{-i \gamma}(i Y)
$$


where $A$ and $B$ are arbitrary constants. Using the group velocity arguments as in section 3.3, we can show that the first solution $A J_{i \gamma}(i Y)$ corresponds to an upward propagating wave while $B J_{-i \gamma}(i Y)$ corresponds to a downward propagating wave. In our study we are interested in upward propagating waves only. So we must have $B=0$.

In this case the solution of (4.14) can be written as a series

$$
\phi \sim \sqrt{z-z_{c}} \sum_{n=0}^{\infty} \frac{(-1)^{n}}{n ! \Gamma(\nu+n+1)}\left[\frac{i \sqrt{\delta} k\left(z-z_{c}\right)}{2}\right]^{2 n+\nu}
$$

The first term in the series is

$$
\phi^{(0)} \sim \sqrt{z-z_{c}} \frac{1}{\Gamma(\nu+1)}\left[\frac{i \sqrt{\delta} k\left(z-z_{c}\right)}{2}\right]^{i \sqrt{R i-1 / 4}}
$$

We note that the solution is proportional to

$$
\phi^{(0)} \sim\left(z-z_{c}\right)^{1 / 2+i \sqrt{R i-1 / 4}}
$$

which coincides with the first term of the solution derived by the Frobenius method in section 4.1 .

\subsection{Solution of the Taylor-Goldstein equation: $\bar{u}$ is a linear function of $z, \delta=0$}

In this section we examine the gravity wave behaviour at the critical level for the case where $\bar{u}(z)$ is a linear function of $z$ and $\delta=0$. In this case (4.3) simplifies to

$$
\left(z-z_{c}\right)^{2} \phi_{z z}+N^{2} \phi=0
$$


This is an Euler-Cauchy equation with solutions of the form $\phi=\left(z-z_{c}\right)^{\alpha}$. Substituting this expression into equation (4.24) we find that

$$
\alpha^{2}-\alpha+N^{2}=0
$$

So, $\alpha=1 / 2 \pm i \gamma$, where $\gamma=\sqrt{N^{2}-1 / 4}=\sqrt{R i-1 / 4}$. And the general solution for the $(4.24)$ is

$$
\phi=A\left(z-z_{c}\right)^{1 / 2+i \gamma}+B\left(z-z_{c}\right)^{1 / 2-i \gamma},
$$

where $\mathrm{A}$ and $\dot{\mathrm{B}}$ are arbitrary constants. As before we consider upward propagating waves which correspond to the plus sign. So we must have $B=0$. The solution $\phi \sim\left(z-z_{c}\right)^{1 / 2+i \gamma}$ corresponds to the first term of each of the solutions obtained in sections 4.1 and 4.2 for the more general configurations. The solution is singular at $z=z_{c}$. The singular behaviour of the solution is qualitatively the same as that of the solutions derived in 4.1 and 4.2. Having assured ourselves of this we will consider this simple case $(\delta=0)$ in most of our analytic discussions from this point on.

Below the critical level, where $z<z_{c}$ the solution can be written as

$$
\left(z-z_{c}\right)^{1 / 2+i \gamma}=\left|z-z_{c}\right|^{1 / 2} e^{i \gamma \log \left|z-z_{c}\right|}
$$

and above the critical level where $z>z_{c}$ we have

$$
\left(z-z_{c}\right)^{1 / 2+i \gamma}=i e^{-\gamma \pi}\left|z-z_{c}\right|^{1 / 2} e^{i \gamma \log \left|z-z_{c}\right|} .
$$

Thus the magnitude of the solution at a given distance from the critical level is not the same above and below it but differs by a factor of $\exp (-\gamma \pi)$, and the phase of the solution changes by $-\pi$. If the solution (4.27) is $O(1)$ below the critical layer then it is 
close to zero above the critical layer. So, we can say that the solution is discontinuous at the critical layer.

From a physical point of view this means that internal gravity waves propagating vertically in a shear flow are almost completely absorbed at the critical level at which the horizontal component of the phase velocity of the wave is equal to the mean velocity of the fluid. The vertical wavenumber becomes shorter in the vicinity of the critical level. In a nonlinear problem the wave absorption would result in a change in the mean flow in the critical layer. This is discussed in Appendix D1. 


\section{Chapter 5}

\section{Gravity waves: solutions of the time-dependent equations with}

$k \neq 0$

In this chapter we will derive solutions of the governing linear time-dependent equation (3.34). In section 5.1 we re-examine the linear inviscid solution to the time-dependent problem obtained by Booker and Bretherton (1967) with a monochromatic forcing $e^{i k x}$. In section 4.2 we generalize the solution to the case in which the forcing is of the form $e^{i k x} e^{-\mu^{2} x^{2}}$. In both sections we shall consider the simplified problem in which $\bar{u}$ is a linear function of $z$. Also we assume without loss of generality that the horizontal phase speed of the wave propagation is zero, $c=0$. We note that assuming that $c \neq 0$ would simply introduce a Doppler-shift into our linear solutions but would not change the qualitative behaviour of the solution. 


\subsection{Linear problem for monochromatic forced waves}

In this section we solve the linear time-dependent problem comprising equation (3.34) with $\bar{u}=z-z_{c}$

$$
\left(\frac{\partial}{\partial t}+\bar{u} \frac{\partial}{\partial x}\right)^{2}\left(\delta \frac{\partial^{2}}{\partial x^{2}}+\frac{\partial^{2}}{\partial z^{2}}\right) \psi+N^{2} \psi_{x x}=0
$$

with $t>0$, in a rectangular domain defined by $0<x<2 \pi, z_{1}<z<\infty$. According to the kinematic boundary conditions a fluid particle does not leave the boundary. So, the displacement of the fluid particle should be proportional to the boundary shape. In our case we solve our problem in rectangular domain. So, to satisfy kinematic boundary conditions corresponding to the real lower boundary (for example, corresponing to a mountain range) we assume a horizontally periodic monochromatic boundary condition at $z=z_{1}$

$$
\psi\left(x, z_{1}, t\right)=e^{i k x}+\text { c.c. }=2 \cos k x
$$

and periodic boundary conditions at $x=0,2 \pi$. The upper boundary condition is that there are no incoming waves from infinity which means that, as before, we consider only upward propagating waves. The boundary condition at $z=z_{1}$ means that the solution takes the form $\psi(x, z, t)=\phi(z, t) e^{i k x}+$ c.c. Note that $\phi$ is complex, but $\psi$ is real. Substituting this into the governing equation (5.1) gives an equation for $\phi$

$$
\left(\frac{\partial}{\partial t}+i k \bar{u}\right)^{2} \phi_{z z}-k^{2}\left(N^{2}+\delta\right) \phi=0
$$

with the boundary condition $\phi\left(z_{1}, t\right)=1$. We solve this equation by making use of the Laplace transform. The solution procedure is described in Appendix A. According to 
(A.27) $\phi(z, t)$ is

$$
\begin{aligned}
\phi(z, t)=\left(\frac{z-z_{c}}{z_{1}-z_{c}}\right)^{1 / 2+i \gamma} & -e^{-i k\left(z-z_{c}\right) t}\left[\frac{G_{1}(z)}{t^{3 / 2+i \gamma}}+\frac{G_{2}(z)}{t^{5 / 2+i \gamma}}\right] \\
& -e^{-i k\left(z_{1}-z_{c}\right) t}\left[\frac{G_{3}(z)}{t^{1 / 2-i \gamma}}+\frac{G_{4}(z)}{t^{3 / 2-i \gamma}}\right]
\end{aligned}
$$

where $G_{1}, G_{2}, G_{3}$ and $G_{4}$ are functions of $z$ which are defined in Appendix A (A.25). This solution consists of a time-independent term which corresponds to the solution of the Taylor-Goldstein equation that was defined in Chapter 4, and some terms proportional to $e^{-i k\left(z-z_{c}\right) t} t^{\alpha}$ where the real part of $\alpha$ is negaive. With the forcing applied at the level $z=z_{1}$, there are two additional time-dependent terms which are proportional to $e^{-i k\left(z_{1}-z_{c}\right) t} t^{\alpha}$. As $t \rightarrow \infty$, all the time dependent terms go to zero and only the steady first term remains. This steady solution corresponds to the first terms of each of the solutions of the Taylor-Goldstein equation which were derived in Chapter 4. With the forcing applied below the critical level, the first term is reduced by a factor of $e^{-\pi \gamma}$ across the critical layer as described in section 4.3 for the steady solution.

\subsection{Nonlinear solution}

The linear solution can be considered as a first approximation to the nonlinear solution. If $\varepsilon$ is nonzero in the governing equations (3.21) and (3.27), then the effects of nonlinearity must be taken into account. Assuming that $\varepsilon \ll 1$, then we can obtain the solution in powers of $\varepsilon$ and the expression for $\psi$ given in (5.4) represents the leading order term in the series. We can write

$$
\psi \sim \psi^{(0)}+\varepsilon \psi^{(1)}+O\left(\varepsilon^{2}\right)
$$


where $\psi^{(1)} \sim O(1)$. When this is substituted into the nonlinear inviscid equations, along with similar expressions for the perturbation density and vorticity, we find that the leading-order nonlinear term is

$$
\varepsilon\left(\psi_{x}^{(0)} \zeta_{z}^{(0)}-\psi_{z}^{(0)} \zeta_{x}^{(0)}\right)=\varepsilon\left(\psi_{x}^{(0)} \psi_{z z z}^{(0)}-\psi_{z}^{(0)} \psi_{z z x}^{(0)}\right)
$$

For large $t, \psi^{(0)} \sim O(1)+O\left(t^{-3 / 2}\right)$ according to (5.4), with the $O\left(t^{-3 / 2}\right)$ term being proportional to $e^{-i k\left(z-z_{c}\right) t}$. Each time $\psi(0)$ is differentiated with respect to $z$, this term is multiplied by a factor of $t$. So,

$$
\begin{aligned}
& \psi_{z}^{(0)} \sim O(1)+O\left(t^{-1 / 2}\right), \\
& \psi_{z z}^{(0)} \sim O(1)+O\left(t^{1 / 2}\right), \\
& \psi_{z z z}^{(0)} \sim O(1)+O\left(t^{3 / 2}\right),
\end{aligned}
$$

therefore, the nonlinear Jacobian is

$$
\left(\psi_{x}^{(0)} \psi_{z z z}^{(0)}-\psi_{z}^{(0)} \psi_{z z x}^{(0)}\right) \sim t^{3 / 2}
$$

which means that the nonlinear terms is $O\left(t^{3 / 2}\right)$. So the nonlinear term becomes $O(1)$ when $t \sim O\left(\varepsilon^{-2 / 3}\right)$ and we can conclude that the linear solution is only valid in the regime where $t<O\left(\varepsilon^{-2 / 3}\right)$. To examine the long-time (fully nonlinear) evolution of the disturbance in the outer region using multiple scaling, one would need to define a slow time scale $T=\varepsilon^{2 / 3} t$. Such an analysis of the late-time nonlinear solution was carried out by Brown and Stewartson (1978). 


\subsection{Wave packet forcing, $\mathrm{k} \neq 0, \delta=0$.}

In this section we generalize Booker and Bretherton's linear analysis to the case in which the forcing takes the form of a wave packet, localized in the horizontal direction and comprising a continuous spectrum of horizontal wave numbers, such as

$$
\psi\left(x, z_{1}, t\right)=A(\mu x) e^{i k x}
$$

where $A \rightarrow 0$ as $x \rightarrow \pm \infty$. Such a boundary condition can be considered as an approximation to a mountain range. The parameter $\mu$ is assumed to be sufficiently small so that the horizontal extent of the mountain range is much greater than the distance between individual peaks and there are several peaks within the mountain range. The case where $A(\mu x)=e^{-\mu^{2} x^{2}}$ is illustrated in Figure 5.1.

The solution procedure follows that employed by Campbell and Maslowe (1998) for the analogous problem for Rossby wave packets. We consider the governing equation (5.1) with $\delta=0$

$$
\left(\frac{\partial}{\partial t}+\bar{u} \frac{\partial}{\partial x}\right)^{2} \psi_{z z}+N^{2} \psi_{x x}=0
$$

with boundary conditions

$$
\psi\left(x, z_{1}\right)=A(\mu x)
$$

As in section 5.1 we consider the case $\bar{u}=z-z_{c}$. We solve the equation by applying the Fourier transform in the $x$ direction and the Laplace transform in $t$. 


\section{Fourier transform}

We define the Fourier transform of $\psi(x, z, t)$ by

$$
\Phi(\lambda, z, t)=\mathcal{F}(\psi(x, z, t))=\int_{-\infty}^{\infty} \psi(x, z, t) e^{-i \lambda x} d x
$$

The Fourier transform of equation (5.8) is

$$
\left(\frac{\partial}{\partial t}+i \lambda \bar{u}\right)^{2} \Phi_{z z}-N^{2} \lambda^{2} \Phi=0
$$

with boundary conditions

$$
\Phi\left(\lambda, z_{1}, t\right)=\mathcal{F}[A(\mu x)]=\tilde{A}(\lambda, \mu) .
$$

For the case where $A(\mu x)=e^{-\mu^{2} x^{2}}$ the boundary condition is

$$
\Phi\left(\lambda, z_{1}, t\right)=\frac{\sqrt{\pi}}{\mu} e^{-(k-\lambda)^{2} / 4 \mu^{2}}
$$

\section{Laplace transform}

We define the Laplace transform of $\Phi(\lambda, z, t)$ by

$$
\tilde{\Phi}(\lambda, z, s)=\int_{0}^{\infty} \Phi(\lambda, z, t) e^{-s t} d t
$$

The Laplace transform of equation (5.11) is

$$
\left(s+i\left(z-z_{c}\right) \lambda\right)^{2} \tilde{\Phi}_{z z}-N^{2} \lambda^{2} \tilde{\Phi}=0 .
$$


with the boundary condition:

$$
\tilde{\Phi}\left(z_{1}, t\right)=\frac{\tilde{A}(\lambda, \mu)}{s}
$$

According to (A.7) the solution is

$$
\tilde{\Phi}(\lambda, z, s)=\tilde{A}(\lambda, \mu) \frac{\left(z-z_{c}-i s / \lambda\right)^{1 / 2+i \gamma}}{\left(z_{1}-z_{c}-i s / \lambda\right)^{1 / 2+i \gamma}} \frac{1}{s}
$$

where

$$
\tilde{A}(\lambda, \mu)=\frac{\sqrt{\pi}}{\mu} e^{-(k-\lambda)^{2} / 4 \mu^{2}}
$$

if the forcing is $e^{-\mu^{2} x^{2}} e^{i k x}$.

We can find the function $\psi(x, z, t)$ by inverting the Laplace and Fourier transforms. The details are described in Appendix B. The solution of our problem is

$$
\begin{array}{r}
\psi=e^{i k x} A(\mu x)\left\{\left(\frac{z-z_{c}}{z_{1}-z_{c}}\right)^{1 / 2+i \gamma}-e^{-i k\left(z-z_{c}\right)}\left[\left(\frac{g_{1}}{(k t)^{3 / 2+i \gamma}}+\frac{g_{2}}{(k t)^{5 / 2+i \gamma}}\right)\right.\right. \\
\left.+\frac{2 \mu^{2} i x}{k}\left(g_{5}(k t)^{1 / 2+i \gamma}+\frac{g_{6}}{(k t)^{3 / 2+i \gamma}}\right)+\frac{\mu^{2}}{k^{2}}\left(g_{9}(k t)^{1 / 2-i \gamma}+\frac{g_{10}}{(k t)^{1 / 2+i \gamma}}\right)\right] \\
-e^{-i k\left(z_{1}-z_{c}\right)}\left[\left(\frac{g_{3}}{(k t)^{1 / 2-i \gamma}}+\frac{g_{4}}{(k t)^{3 / 2-i \gamma}}\right)+\frac{2 \mu^{2} i x}{k}\left(g_{7}(t k)^{1 / 2-i \gamma}+\frac{g_{8}}{(k t)^{1 / 2-i \gamma}}\right)\right. \\
\left.\left.+\frac{\mu^{2}}{k^{2}}\left(g_{11}(k t)^{3 / 2+i \gamma}+g_{12}(k t)^{1 / 2+i \gamma}\right)\right]\right\} .
\end{array}
$$

where the functions $g_{1}(z)-g_{12}(z)$ are defined by expressions (B.14), (B.15) and (B.17) in Appendix B. In the limit as $\mu \rightarrow 0$ the monochromatic solution (5.4) is recovered. The $O\left(\mu^{2}\right)$ term of the solution grows with time. To get a bounded solution we have to take into consideration nonlinear terms. This problem is included in our plans for future work. In the numerical simulation which is described in Chapter 7 the solution for the the nonlinear case was obtained, and it appears to be bounded. 


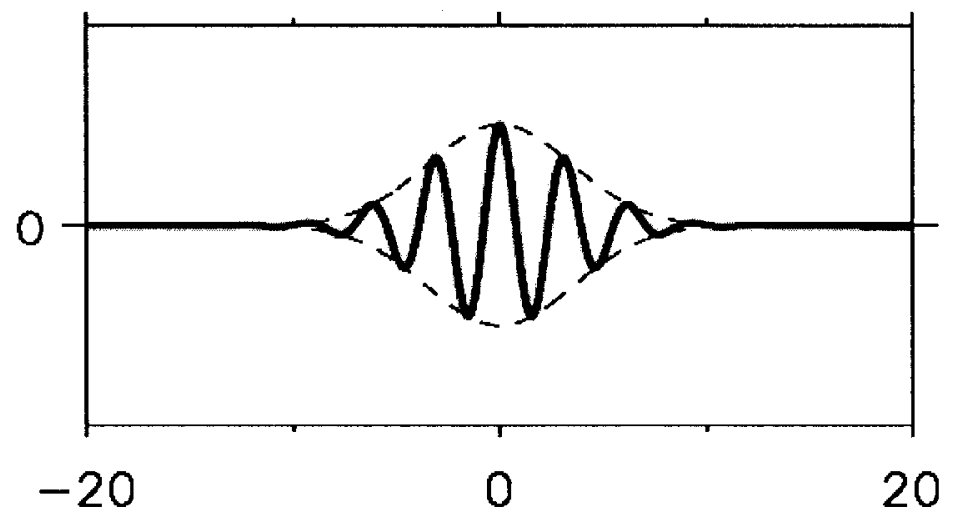

Figure 5.1: Boundary conditions in the form of a wave packet $\psi=e^{-\mu^{2} x^{2}} e^{i k x}$ with wavenumber $k$ and length $\sim \mu^{-1}$. 


\section{Chapter 6}

\section{Gravity waves forced by an isolated mountain $(k=0$ : analytical}

\section{solution)}

Now we consider the case when the gravity waves packet is forced by some isolated topography, i.e. $k=0$ in the forcing function (5.7). We will solve the time-dependent equation (5.1) with a boundary condition which corresponds to an isolated mountain.

For example, if $A(\mu x)=e^{-\mu^{2} x^{2}}$ then the "mountain" takes the form shown in Figure 6.1 .

In the previous chapter we solved the linear governing equation with the boundary condition $A(\mu x) e^{i k x}$ and obtained the solution (5.19). Now we set $k=0$. The solution (5.19) is singular if $k \rightarrow 0$ and we cannot use it for the case of an isolated mountain. To obtain an approximate solution to this problem, we use the method of multiple scales. We define a so-called "slow scale" $X=\mu x$ that corresponds to the scale of the topography and to the length of the forced wave packet. In general, if $k \neq 0$, we also have the usual "fast scale" $x$ that corresponds to the wavelength of oscillations in the 
packet. The boundary condition at $z=z_{1}$ can be written in terms of the two scales as $A(X) e^{i k x}$. To express equation (5.3) in terms of the two scales, each $x$-derivative is replaced by the linear operator $i k+\mu \frac{\partial}{\partial X}$. In this chapter we consider the case $k=0$ so we have only the large scale $X$. Each $x$-derivative in equation (5.3) is replaced by $\mu \frac{\partial}{\partial X}$. So, equation (5.1) becomes

$$
\left(\frac{\partial}{\partial t}+\mu \bar{u} \frac{\partial}{\partial X}\right)^{2}\left(\psi_{z z}+\delta \mu^{2} \psi_{X X}\right)+\mu^{2} N^{2} \psi_{X X}=0
$$

We solve the problem for the general case where the boundary condition is arbitrary function of $X$, i.e.

$$
\psi\left(X, z_{1}\right)=A(X)
$$

where $A(X) \rightarrow 0$ as $X \rightarrow \pm \infty$. The goal is to solve the time-dependent equation (6.1), but before doing that let us first consider the corresponding steady problem.

\subsection{Steady state case}

We begin our multiple scale analysis by considering a steady case in which the gravity waves do not depend on time. In general, with $k \neq 0$, we would seek a solution of the form

$$
\psi(x, X, z)=\phi(X, z) e^{i k x}+\text { c.c. }
$$

With $k=0$ the solution is simply $\phi(X, z)$. As before we impose a radiation condition at the upper boundary, so we consider only upward propagating waves. 


\section{The case $\bar{u}=$ constant (no shear)}

Let us start with the simplest case in which $k=0$ (isolated mountain) and $\bar{u}=$ constant. According to (5.1) the steady state equation under these assumptions is

$$
\bar{u}^{2}\left(\delta \mu^{2} \frac{\partial^{2}}{\partial X^{2}}+\frac{\partial^{2}}{\partial z^{2}}\right) \phi_{X X}+N^{2} \phi_{X X}=0
$$

Note that with $\bar{u}=$ constant there is no singularity and hence no critical level. With $\delta=0$ the equation becomes

$$
\bar{u}^{2} \phi_{X X z z}^{(0)}+N^{2} \phi_{X X}^{(0)}=0
$$

and the solution satisfying the boundary conditions $\phi\left(X, z_{1}\right)=A(X)$, where $A \rightarrow 0$ as $x \rightarrow \pm \infty$ and the condition of only upward propagation, is simply

$$
\phi(X, z)=A(X) e^{\frac{i N}{\bar{u}}\left(z-z_{1}\right)} .
$$

In that case the solution is periodic in the $z$ direction with vertical wavenumber $N / \bar{u}$ and at all levels it maintains the horizontal profile $A(X)$ which is specified at the boundary $z=z_{1}$. Since there is no critical level, the waves propagate up to infinity without being absorbed.

For the case $\delta \neq 0$ the form of equation (6.4) suggests that we can find a solution in powers of the small parameter $\delta \mu^{2}$ :

$$
\phi(X, z)=\phi^{(0)}(X, z)+\delta \mu^{2} \phi^{(1)}(X, z)+\delta^{2} \mu^{4} \phi^{(2)}(X, z)+O\left(\delta^{3} \mu^{6}\right) .
$$

Substituting the series (6.7) into equation (6.4) and grouping terms of orders $O(1)$, 
$O\left(\delta \mu^{2}\right)$ and so on, gives equations

$$
\begin{aligned}
& O(1): \bar{u}^{2} \phi_{X X z z}^{(0)}+N^{2} \phi_{X X}^{(0)}=0, \\
& O\left(\delta \mu^{2}\right): \bar{u}^{2} \phi_{X X X X}^{(0)}+\bar{u}^{2} \phi_{X X z z}^{(1)}+N^{2} \phi_{X X}^{(1)}=0 .
\end{aligned}
$$

Integrating equation (6.8) twice with respect to $X$ and applying the zero boundary conditions on $\phi^{(0)}$ and its derivatives as $X \rightarrow \pm \infty$ we get

$$
\bar{u}^{2} \phi_{z z}^{(0)}+N^{2} \phi^{(0)}=0
$$

There are two solutions for equation $(6.10), \phi^{(0)} \sim e^{ \pm \frac{i N}{\bar{u}}\left(z-z_{1}\right)}$, and we consider the solution with the plus sign which corresponds to upward propagating waves. Thus the solution for $\phi^{(0)}$ satisfying the boundary condition is

$$
\phi^{(0)}=A(X) \exp \left(\frac{i N}{\bar{u}}\left(z-z_{1}\right)\right)
$$

Substituting $\phi^{(0)}$ into equation (6.9) we get an equation for $\phi^{(1)}$ :

$$
\bar{u}^{2} \phi_{X X z z}^{(1)}+N^{2} \phi_{X X}^{(1)}=-\bar{u}^{2} A_{X X X X} \exp \left(\frac{i N}{\bar{u}}\left(z-z_{1}\right)\right)
$$

Integrating (6.12) twice with respect to $X$ and again applying the zero boundary conditions as $X \rightarrow \pm \infty$ gives

$$
\phi_{z z}^{(1)}+\frac{N^{2}}{\bar{u}^{2}} \phi^{(1)}=-A_{X X} \exp \left(\frac{i N}{\bar{u}}\left(z-z_{1}\right)\right)
$$

The general solution of equation (6.13) is the sum of the general solution to the related homogeneous equation and a particular solution of the inhomogeneous equation. The 
general solution of the homogeneous equation is of the same form as $\phi^{(0)}$ :

$$
\phi_{\mathrm{hom}}^{(1)}=C(X) \exp \left(i \frac{N}{\bar{u}}\left(z-z_{1}\right)\right) .
$$

To solve the inhomogeneous equation we search for a solution of the form

$$
C_{1}(X)\left(z-z_{1}\right) \exp \left(\frac{i N}{\bar{u}}\left(z-z_{1}\right)\right)
$$

After substituting it in equation (6.13) we find that

$$
C_{1}(X)=\frac{i \bar{u}}{2 N}
$$

The final solution for the steady-state case is

$$
\phi(X, z)=e^{\left(i \frac{N}{\bar{u}}\left(z-z_{1}\right)\right)}\left[A(X)+\frac{i \bar{u}}{2 N} \delta \mu^{2} A_{X X}\left(z-z_{1}\right)+O\left(\delta^{2} \mu^{4}\right)\right]
$$

It can be shown that the $O\left(\delta^{2} \mu^{4}\right)$ term is proportional to the product of $\left(z-z_{1}\right)^{2}$ and the 4 th derivative of $A(X)$.

So for the case $\delta \neq 0$ the effect of the terms of order $\delta \mu^{2}, \delta^{2} \mu^{4}$ and higher is to modify the horizontal profile of the solution. For example if the boundary function is $A(X)=e^{-X^{2}}$ then the higher order terms in the solution are proportional to $A_{X X}=\left(-2+4 X^{2}\right) e^{-X^{2}}, A_{X X X X}=\left(12-48 X^{2}+16 X^{4}\right) e^{-X^{2}}$ and so on.

The case where $\bar{u}$ is a linear function, $\bar{u}=z-z_{c}$

Now we consider the solution of the steady equation (6.4)

$$
\bar{u}^{2}\left(\delta \mu^{2} \frac{\partial^{2}}{\partial X^{2}}+\frac{\partial^{2}}{\partial z^{2}}\right) \phi_{X X}+N^{2} \phi_{X X}=0
$$


for the case where there is wind shear and the mean flow is a linear function of $z$ : $\bar{u}=z-z_{c}$. As before, the boundary condition at $z=z_{1}$ is

$$
\phi\left(X, z_{1}\right)=A(X)
$$

and we look for a solution of (6.17) of the form

$$
\phi(X, z, t)=\phi^{(0)}(X, z, t)+\delta \mu^{2} \phi^{(1)}(X, z, t)+\delta^{2} \mu^{4} \phi^{(2)}(X, z, t)+O\left(\delta^{3} \mu^{6}\right)
$$

where $\phi^{(0)}$ and $\phi^{(1)}$ satisfy equations (6.8) and (6.9). For $\phi^{(0)}$ we have the equation

$$
\bar{u}^{2} \phi_{X X z z}^{(0)}+N^{2} \phi_{X X}^{(0)}=0
$$

which can be integrated to give

$$
\bar{u}^{2} \phi_{z z}^{(0)}+N^{2} \phi^{(0)}=0
$$

since $\phi^{(0)} \rightarrow 0$ as $X \rightarrow \pm \infty$. The solution satisfying the boundary condition at $z=z_{1}$ is

$$
\phi^{(0)}=A(X)\left(\frac{z-z_{c}}{z_{1}-z_{c}}\right)^{1 / 2+i \gamma}
$$

where $\gamma=\sqrt{N^{2}-1 / 4}$. Substituting $\phi^{(0)}$ into the equation for $\phi^{(1)}$

$$
\bar{u}^{2} \phi_{X X X X}^{(0)}+\bar{u}^{2} \phi_{X X z Z}^{(1)}+N^{2} \phi_{X X}^{(1)}=0
$$

we have

$$
\left(z-z_{c}\right)^{2} \phi_{X X z z}^{(1)}+N^{2} \phi_{X X}^{(1)}=-A_{X X X X} \frac{\left(z-z_{c}\right)^{5 / 2+i \gamma}}{\left(z_{1}-z_{c}\right)^{1 / 2+i \gamma}}
$$


Integrating (6.24) twice with respect to $X$ and assuming as usual that $\phi^{(1)} \rightarrow 0$ as $X \rightarrow \pm \infty$ we get

$$
\phi_{z z}^{(1)}+\frac{N^{2}}{\left(z-z_{c}\right)^{2}} \phi^{(1)}=-A_{X X} \frac{\left(z-z_{c}\right)^{1 / 2+i \gamma}}{\left(z_{1}-z_{c}\right)^{1 / 2+i \gamma}}
$$

As before, the general solution of equation (6.25) is the sum of the general solution to the related homogeneous equation and a particular solution of an inhomogeneous equation. The two solutions of the homogeneous equation are similar to the solutions for $\phi^{(0)}$ :

$$
\begin{aligned}
& \bar{F}_{1}=\left(z-z_{c}\right)^{1 / 2+i \gamma} \\
& \bar{F}_{2}=\left(z-z_{c}\right)^{1 / 2-i \gamma}
\end{aligned}
$$

To find a particular solution of the inhomogeneous equation we use the method of variation of parameters and look for a solution of the form

$$
\phi_{p}^{(1)}=V_{1} \bar{F}_{1}+V_{2} \bar{F}_{2}
$$

where $V_{1}$ and $V_{2}$ are functions of $z$ which satisfy

$$
\frac{d V_{1}}{d z}=\frac{A_{X X} \bar{F}_{2}\left(z-z_{c}\right)^{1 / 2+i \gamma}}{W\left(z_{1}-z_{c}\right)^{1 / 2+i \gamma}}
$$

and

$$
\frac{d V_{2}}{d z}=-\frac{A_{X X} \bar{F}_{1}\left(z-z_{c}\right)^{1 / 2+i \gamma}}{W\left(z_{1}-z_{c}\right)^{1 / 2+i \gamma}}
$$

where $W$ is the Wronskian determinant. For our system of solutions the Wronskian $W$ is

$$
W=\bar{F}_{1} \frac{d \bar{F}_{2}}{d z}-\bar{F}_{2} \frac{d \bar{F}_{1}}{d z}=-2 i \gamma
$$


So,

$$
\begin{gathered}
\frac{d V_{1}}{d z}=-\frac{A_{X X}\left(z-z_{c}\right)}{2 i \gamma\left(z_{1}-z_{c}\right)^{1 / 2+i \gamma}} \\
\frac{d V_{2}}{d z}=\frac{A_{X X}\left(z-z_{c}\right)^{1+2 i \gamma}}{2 i \gamma\left(z_{1}-z_{c}\right)^{1 / 2+i \gamma}}
\end{gathered}
$$

and,

$$
\begin{gathered}
V_{1}=-\frac{A_{X X}\left(z-z_{c}\right)^{2}}{4 i \gamma\left(z_{1}-z_{c}\right)^{1 / 2+i \gamma}}, \\
V_{2}=\frac{A_{X X}\left(z-z_{c}\right)^{2+2 i \gamma}}{4 i \gamma(1+i \gamma)\left(z_{1}-z_{c}\right)^{1 / 2+i \gamma}} .
\end{gathered}
$$

So, the particular solution to $(6.25)$ is

$$
\phi_{p}^{(1)}=-\frac{A_{X X}\left(z-z_{c}\right)^{5 / 2+i \gamma}}{4(1+i \gamma)\left(z_{1}-z_{c}\right)^{1 / 2+i \gamma}}
$$

And the general solution for $\phi_{X X}^{(1)}$ of $(6.25)$ is

$$
\phi^{(1)}=C(X)\left(z-z_{c}\right)^{1 / 2+i \gamma}-\frac{A_{X X}\left(z-z_{c}\right)^{5 / 2+i \gamma}}{4(1+i \gamma)\left(z_{1}-z_{c}\right)^{1 / 2+i \gamma}}
$$

Applying the boundary conditions $\phi_{X}^{(1)}\left(X, z_{1}\right)=0$ we get the solution for the steady state case:

$$
\phi^{(1)}=\frac{A_{X X}}{4(1+i \gamma)\left(z_{1}-z_{c}\right)^{1 / 2+i \gamma}}\left[\left(z_{1}-z_{c}\right)^{2}\left(z-z_{c}\right)^{1 / 2+i \gamma}-\left(z-z_{c}\right)^{5 / 2+i \gamma}\right]
$$

The final solution for the steady state case is

$$
\begin{aligned}
& \phi(X, z)=A(X)\left(\frac{z-z_{c}}{z_{1}-z_{c}}\right)^{1 / 2+i \gamma}+ \\
& \delta \mu^{2} \frac{A_{X X}\left(z-z_{c}\right)^{1 / 2+i \gamma}}{4(1+i \gamma)\left(z_{1}-z_{c}\right)^{1 / 2+i \gamma}}\left[\left(z_{1}-z_{c}\right)^{2}-\left(z-z_{c}\right)^{2}\right]+O\left(\delta^{2} \mu^{4}\right) .
\end{aligned}
$$


The leading order term is the solution that would be obtained if $\delta=0$, which is the same steady singular solution that was derived in Chapter 4.

So $\phi^{(1)} \sim A_{X X}$ and it can be shown that the $\phi^{(2)}$ term of order $O\left(\delta^{2} \mu^{4}\right)$ is proportional to $A_{X X X X}$ and so on. So, again, the effect of specifying $\delta \neq 0$ is to modify the horizontal profile of the solution. A contour plot of this steady solution is shown in Figure 6.2. It was computed in a rectangular domain $0<z<10$ and $-20<x<20$ for the parameter values: $N=2.0, \delta=0.1$ and $\mu=0.2$. The mean flow is $\bar{u}=z-z_{c}$ where the height of the critical level $z_{c}=5$. The contour plot shows that the vertical wavelength decreases as the wave packet approaches the critical level and the horizontal structure of the solution is modified from its profile at the lower boundary.

\subsection{Time-dependent solution}

A more realistic representation of the solution is obtained by allowing the solution to vary with time. Given the boundary condition $\psi(x, X, z, t)=A(X) e^{i k x}$ we seek a solution for (5.3) of the form $\psi(x, X, z, t)=\phi(X, z, t) e^{i k x}$. For the case of isolated topography we set $k=0$ and we have only one spatial variable, the slow scale $X$ and there is no fast variable. The $x$-derivative becomes $\mu \frac{\partial}{\partial X}$. For the case of the linear function $\bar{u}=z-z_{c}$ the governing equation is

$$
\left(\frac{\partial}{\partial t}+\bar{u} \mu \frac{\partial}{\partial X}\right)^{2}\left(\delta \mu^{2} \frac{\partial^{2}}{\partial X^{2}}+\frac{\partial^{2}}{\partial z^{2}}\right) \phi+\mu^{2} N^{2} \frac{\partial^{2} \phi}{\partial X^{2}}=0
$$

To solve (6.40) we look for a solution of the form

$$
\phi(X, z, t)=\phi^{(0)}(X, z, t)+\mu \phi^{(1)}(X, z, t)+\mu^{2} \phi^{(2)}(X, z, t)+O\left(\delta \mu^{2}\right) .
$$


Substituting the series (6.41) into equation (6.40) gives three equations of orders $O(1)$, $O(\mu)$ and $O\left(\mu^{2}\right)$ :

$$
\begin{aligned}
& O(1): \quad \phi_{z z t t}^{(0)}=0 \\
& O(\mu): \quad \phi_{z z t t}^{(1)}=-2 \bar{u} \phi_{z z X t}^{(0)}, \\
& O\left(\mu^{2}\right): \quad \phi_{z z t t}^{(2)}=-\bar{u}^{2} \phi_{z z X X}^{(0)}-2 \bar{u} \phi_{z z t X}^{(1)}-N^{2} \phi_{X X}^{(0)} .
\end{aligned}
$$

Equation (6.42) is solved by direct integration to give

$$
\phi^{(0)}=F_{0}(X, z)+B_{0}(X, t)\left(z-z_{1}\right)+t g_{0}(X, z)
$$

where $B_{0}(X, t)$ and $g_{0}(X, z)$ are functions that can be determined by applying the boundary condition $\phi^{(0)}\left(X, z_{1}, t\right)=A(X)$. Thus

$$
F_{0}\left(X, z_{1}\right)=A(X) ; \quad g_{0}(x, z)=0 .
$$

This means that the $O(\mu)$ equation is

$$
\phi_{z z t t}^{(1)}=-2 \bar{u} \phi_{x z z t}^{(0)}=0
$$

After integrating we have

$$
\phi^{(1)}=F_{1}(X, z)+B_{1}(X, t)\left(z-z_{1}\right)+t g_{1}(x, z)
$$


with the functions $B_{1}(X, t)$ and $g_{1}(X, z)$ that can be determined by applying the boundary condition $\phi^{(1)}\left(X, z_{1}\right)=0$ :

$$
F_{1}\left(X, z_{1}\right)=0, \quad g_{1}(x, z)=0
$$

Now, substituting solutions (6.45) and (6.48) into (6.44),

$$
\left(\bar{u}^{2} \frac{\partial^{2}}{\partial z^{2}}+N^{2}\right) \phi_{X X}^{(0)}+2 \bar{u} \phi_{X z z t}^{(1)}+\phi_{z z t t}^{(2)}=0
$$

we obtain an equation for $\phi^{(2)}$

$$
\phi_{z z t t}^{(2)}=-N^{2} B_{0}(X, t)\left(z-z_{1}\right)-\bar{u}^{2} F_{0 z z X X}-N^{2} F_{0 X X}
$$

The last two terms on the right-hand side of (6.51) do not depend on $t$. So, if after integrating by $t$ we want to get a solution that is bounded when $t \rightarrow \infty$, we have to demand that

$$
\bar{u}^{2} F_{0 z z X X}+N^{2} F_{0 X X}=0
$$

This is called a secularity condition. If $\bar{u}$ is a linear function, $\bar{u}=z-z_{c}$, we have an equation for $F_{0}$,

$$
\left(z-z_{c}\right)^{2} F_{0 z z X X}+N^{2} F_{0 X X}=0
$$

and its solution satisfying the boundary conditions at $z=z_{1}$ and the limiting conditions at $x \rightarrow \pm \infty$ is

$$
F_{0}(X, z)=A(X)\left(\frac{z-z_{c}}{z_{1}-z_{c}}\right)^{1 / 2+i \gamma}
$$


where $\gamma=\sqrt{N^{2}-1 / 4}$. So, the first term of the time-dependent solution coincides with the first term of the steady-state solution (6.39). The solution of (6.51) is

$$
\phi^{(2)}=B_{2}(X, t)\left(z-z_{1}\right)^{3} / 6+g_{2}(X, z) t+F_{2}(X, z)
$$

where

$$
B_{2 t t}(X, t)=-N^{2} B_{0 X X}
$$

and, according to the boundary condition,

$$
g_{2}(X, z)=0, \quad F_{2}\left(X, z_{1}\right)=0
$$

For $\phi^{(3)}$ we obtain the equation

$$
\begin{aligned}
& \phi_{z z t t}^{(3)}=-2 \bar{u} B_{2 X t}(X, t)\left(z-z_{1}\right) \\
& -\bar{u}^{2} F_{1 z z}(X, z)-N^{2}\left(F_{1}(X, z)+B_{1}(X, t)\left(z-z_{1}\right) .\right.
\end{aligned}
$$

To get a bounded solution after integrating with respect to time we must impose the secularity condition

$$
\left(z-z_{c}\right)^{2} F_{1 z z x x}+N^{2} F_{1 x x}=0 .
$$

The solution $F_{1}$ is proportional to $\left(z-z_{1}\right)^{p}$, and according to the boundary condition $F_{1}\left(x, z_{1}\right)=0$ we can conclude that

$$
F_{1}(X, z)=0
$$


In a similar way we can show that

$$
F_{2}(X, z)=0
$$

Finally, putting all the terms together we obtain

$$
\begin{aligned}
\phi(X, z)=A(X)\left(\frac{z-z_{c}}{z_{1}-z_{c}}\right)^{1 / 2+i \gamma}+ & B_{0}(X, t)\left(z-z_{1}\right)+\mu B_{1}(X, t)\left(z-z_{1}\right)+ \\
& \mu^{2} B_{2}(X, t)\left(z-z_{1}\right)^{3} / 6+O\left(\mu^{3}\right)+O\left(\delta \mu^{2}\right) .
\end{aligned}
$$

The leading term in the solution consists of the steady-state term and the timedependent term $B_{0}(X, t)\left(z-z_{1}\right)$. In our solution (6.62) we have the arbitrary functions $B_{0}, B_{1}$ and $B_{2}$. To find these functions we need some additional conditions. For example, we can consider the area close to the critical level, where $\left|z-z_{c}\right|<\Delta$, which is called an inner layer. Using the solution in the inner layer we can find the arbitrary functions. In the next section the inner layer solution will be used to find $B_{0}(X, t)$. The matching conditions show that $B_{0}(X, t) \rightarrow 0$ as $t \rightarrow \infty$ and the solution tends to a steady state.

\subsection{Inner solution. Linear case}

In the vicinity of the critical level where $z \rightarrow z_{c}$ our solution $\psi \sim\left(z-z_{c}\right)^{1 / 2} e^{i \gamma \log \left(z-z_{c}\right)}$ $\rightarrow 0$, but its derivatives $\psi_{z} \sim\left(z-z_{c}\right)^{-1 / 2+i \gamma}$ and $\psi_{z z} \sim\left(z-z_{c}\right)^{-3 / 2+i \gamma}$ are singular. Thus the horizontal velocity $u=-\psi_{z}$ and the vorticity $\zeta \sim \psi_{z z}$ are also singular at $z=z_{c}$. So, to get a nonsingular solution we have to consider a so-called inner layer at $z \rightarrow z_{c}$. We look for an inner solution that is nonsingular at $z=z_{c}$. In the previous 
section we derived the outer solution for

$$
\psi^{(0)}=A(X)\left(\frac{z_{c}-z}{z_{c}-z_{1}}\right)^{1 / 2+i \gamma}+B_{0}(X, t)\left(z-z_{1}\right)
$$

We write $z-z_{1}=\left(z-z_{c}\right)+\left(z_{c}-z_{1}\right)$ so that

$$
\psi^{(0)}=A(X)\left(\frac{z_{c}-z}{z_{c}-z_{1}}\right)^{1 / 2+i \gamma}+\left(z-z_{c}\right) B_{0}(X, t)+\left(z_{c}-z_{1}\right) B_{0}(X, t) .
$$

In the vicinity of the critical layer $\left|z-z_{c}\right| \ll 1$, so the leading order term is $\left(z_{c}-z_{1}\right) B_{0}(X, t) \sim O(1)$ followed by the first term $\sim O\left(z-z_{c}\right)^{1 / 2}$ and the second term $\sim O\left(z-z_{c}\right)$. This tells us that the inner solution should also take the form

$$
\psi_{\text {inner }} \sim O(1)+O\left(\left(z-z_{c}\right)^{1 / 2}\right)+O\left(z-z_{c}\right) .
$$

It is the $O\left(\left(z-z_{c}\right)^{1 / 2}\right)$ term that is singular at $z=z_{c}$. To find a nonsingular inner solution we must chose $B(X, t)$ in the $\mathrm{O}(1)$ term so that it cancels out the singular second term on the boundary between the outer and inner solutions

$$
A(X)\left(\frac{z_{c}-z}{z_{c}-z_{1}}\right)^{1 / 2+i \gamma}+\left(z_{c}-z_{1}\right) B_{0}(X, t)=0
$$

The choice $B_{0}(X, t)$ that will cancel the singular term is

$$
B_{0}(X, t)=\frac{A(X)}{\left(z_{1}-z_{c}\right)^{3 / 2+i \gamma}} t^{\alpha}
$$

where $\alpha$ is an approximately chosen complex constant. Since we want the solution to be bounded as $t \rightarrow \infty$, the real part of $\alpha$ is negative.

If we define a critical layer of thickness $\left|z-z_{c}\right| \sim O\left(t^{-1}\right)$ the singular term is 
cancelled out and the outer and inner solutions match as $\left|z-z_{c}\right| \rightarrow t^{-1}$, if $\alpha$ is chosen to be $1 / 2+i \gamma$, i.e. if

$$
B_{0}(X, t)=\frac{A(X)}{\left(z_{1}-z_{c}\right)^{3 / 2+i \gamma}} t^{-(1 / 2+i \gamma)}
$$

Thus, the nonsingular inner solution is

$$
\psi_{\mathrm{inner}}^{(0)} \sim\left(z-z_{c}\right) B_{0}(X, t)=A(X) \frac{\left(z-z_{c}\right)}{\left(z_{1}-z_{c}\right)^{3 / 2+i \gamma}} t^{-(1 / 2+i \gamma)}
$$

Substituting (6.68) into (6.63) gives the outer solution

$$
\psi_{\text {outer }}^{(0)}=A(X)\left(\frac{z-z_{c}}{z_{1}-z_{c}}\right)^{1 / 2+i \gamma}+A(X) \frac{\left(z-z_{1}\right)}{\left(z_{1}-z_{c}\right)^{3 / 2+i \gamma}} t^{-(1 / 2+i \gamma)}
$$

The inner solution is defined for $\left|z-z_{c}\right|<O\left(t^{-1}\right)$ and the outer solution is defined for $\left|z-z_{c}\right|>O\left(t^{-1}\right)$.

So, in this section we derived the linear leading term of the inner solution for the linear case, and also found the unknown function for the leading term of the outer solution.

\subsection{Nonlinear case}

In the previous section we got a linear solution for the inner layer. In this solution the width of the inner layer is proportional to $t^{-1}$. By matching with the inner solution we found that the outer solution is of the form

$$
\Phi \sim A(X) h(z)+B_{0}(X, t)\left(z-z_{1}\right)
$$


where

$$
h(z)=\left(\frac{z-z_{c}}{z_{1}-z_{c}}\right)^{1 / 2+i \gamma}, \quad B_{0}(X, t)=\frac{A(X)}{\left(z_{1}-z_{c}\right)^{3 / 2+i \gamma}} t^{-(1 / 2+i \gamma)}
$$

In this section we will examine the corresponding nonlinear problem using this linear solution as a starting point.

The governing equation for the nonlinear case was derived in Chapter 3:

$$
\begin{array}{r}
\left(\frac{\partial}{\partial t}+\bar{u} \frac{\partial}{\partial x}\right)^{2}\left(\psi_{z z}+\delta \psi_{x x}\right)+N^{2} \psi_{x x}= \\
-\varepsilon\left(\frac{\partial}{\partial t}+\bar{u} \frac{\partial}{\partial x}\right)\left(\psi_{x} \psi_{z z z}-\psi_{z} \psi_{z z x}\right)
\end{array}
$$

We consider this equation with the same boundary condition as before $\psi\left(x, z_{1}, t\right)=$ $A(\mu x)$ with $A \rightarrow 0$ as $x \rightarrow \pm \infty$. In the nonlinear problem, there are two small parameters: $\mu$ which determines the width of the mountain and $\varepsilon$ which determines the height of the mountain. In terms of the large-scale variable $X=\mu x$ equation $(6.73)$ is

$$
\begin{array}{r}
\left(\frac{\partial}{\partial t}+\mu \bar{u} \frac{\partial}{\partial X}\right)^{2}\left[\psi_{z z}+O\left(\delta \mu^{2}\right)\right]+\mu^{2} N^{2} \psi_{X X}= \\
-\mu \varepsilon\left(\frac{\partial}{\partial t}+\mu \bar{u} \frac{\partial}{\partial X}\right)\left(\psi_{X} \psi_{z z z}-\psi_{z} \psi_{z z X}\right)+O\left(\varepsilon \delta \mu^{2}\right) .
\end{array}
$$

We look for a solution in powers of $\mu$ and $\varepsilon$, and the first few terms are

$$
\psi(X, z, t)=\Phi^{(0)}+\mu \Phi^{(1)}+\mu \varepsilon \Phi^{(2)}+O\left(\mu^{2}\right) .
$$

Since $\varepsilon$ only appears in equation (6.74) multiplied by $\mu$, but does not appear by itself we do not include an $O(\varepsilon)$ term in the series in (6.75). Depending of the relative 
magnitude of $\mu$ and $\varepsilon$ we can have different solutions. We will consider four cases:

- If $\varepsilon$ is very small, $\varepsilon \lesssim \mu^{2}$, then we can use the solution for the linear problem at least up to $O\left(\mu^{2}\right)$ because the problem depends on $\varepsilon$ only in the higher order terms, and up to $O\left(\mu^{2}\right)$ we can consider this case as the linear case. The solution for this case was derived in the previous section.

- If $\varepsilon \sim O(\mu)$ then the nonlinear $O(\mu \varepsilon)$ terms in (6.74) are $O\left(\mu^{2}\right)$, the problem is not linear, but we can use the same analytical form of the solution for the linear problem up to $O(\mu)$. At $O\left(\mu^{2}\right)$ we must include the nonlinear terms in (6.74). So, in (6.75) the third and the fourth terms on the right-hand side, $\mu^{2} \Phi^{(3)}$ and $\varepsilon \mu \Phi^{(2)}$, are of the same order and can be grouped in one term.

- If $\varepsilon \sim O(1)>>\mu$ the problem is strongly nonlinear. The second and third terms on the right-hand side of $(6.75), \mu \Phi^{(1)}$ and $\varepsilon \mu \Phi^{(2)}$, are of the same order and can be grouped in one term. We cannot find a solution in powers of $\mu$ using the linear solution as a starting point. In this case the $O(\mu)$ equation would be nonlinear.

- If $\mu^{2}<\varepsilon<\mu \ll 1$ then we can look for a solution of the form (6.75) with $\mu^{3} \ll \varepsilon \mu \ll \mu^{2}$. Let us examine this case further.

So, we examine the solution for the nonlinear case where $\mu^{2}<\varepsilon<\mu$. After substituting the solution (6.75) into (6.74) we get

$$
\begin{aligned}
& O(1): \Phi_{z z t t}^{(0)}=0 \\
& O(\mu): \Phi_{z z t t}^{(1)}=-2 \bar{u} \Phi_{t X z z}^{(0)} \\
& O(\mu \varepsilon): \Phi_{z z t t}^{(2)}=-\frac{\partial}{\partial t}\left(\Phi_{X}^{(0)} \Phi_{z z z}^{(0)}-\Phi_{z}^{(0)} \Phi_{z z X}^{(0)}\right) .
\end{aligned}
$$


For $\Phi^{(0)}$ we have the same equation as for the linear case. So the solution is

$$
\Phi^{(0)}=A(X) h(z)+B_{0}(X, t)\left(z-z_{1}\right)
$$

where

$$
h(z)=\left(\frac{z-z_{c}}{z_{1}-z_{c}}\right)^{1 / 2+i \gamma}, \quad B_{0}(X, t)=\frac{A(X)}{\left(z_{1}-z_{c}\right)^{3 / 2+i \gamma}} t^{-(1 / 2+i \gamma)}
$$

Substituting this solution into (6.76) we get an equation for $\Phi^{(1)}$ :

$$
\Phi_{z z t t}^{(1)}=0 .
$$

With the zero boundary condition $\Phi^{(1)}(x, z, t)=0$, the solution of this equation is $\Phi^{(1)}=0$. So, the nonlinear effects appear only in $\Phi^{(2)}$, in the terms of order $O(\mu \varepsilon)$. From (6.76) we have an equation for $\Phi^{(2)}$ :

$$
\Phi_{z z t}^{(2)}=-A A_{X}\left(h h_{z z z}-h_{z} h_{z z}\right)+B_{0} A_{X} h_{z z}-B_{0 X} A h_{z z z}\left(z-z_{1}\right)
$$

To solve this equation we first integrate it with respect to $t$ to obtain $\phi_{z z}^{(2)}$. The terms $-A A_{X}\left(h h_{z z z}-h_{z} h_{z z}\right)$ on the right-hand side are independent of $t$ while the remaining terms of the right-hand side are proportional to $t^{-(1 / 2+i \gamma)}$. This means that after integration with respect to $t$, the function

$$
\phi_{z z}^{(2)} \sim O(t)+O\left(t^{1 / 2}\right)
$$

and it becomes unbounded as $t \rightarrow \infty$. Thus the nonlinear solution is

$$
\psi \sim \Phi^{(0)}+O(\mu \varepsilon t)
$$


and when $t \sim O\left(\mu^{-1} \varepsilon^{-1}\right)$ the nonlinear term is of order $O(1)$. This means than in order to examine the solution for $t>\mu^{-1} \varepsilon^{-1}$ we need to introduce a late-time variable $T=\mu \varepsilon t$. We will consider this case in the next section. It is interesting to note that in this configuration $(k=0)$ the nonlinear time scale is $\mu^{-1} \varepsilon^{-1}$ and depends on both the height and width of the mountain. This is in contrast to the $k \neq 0$ case considered by earlier researchers (section 5.2 ) where the nonlinear time scale is $\sim \varepsilon^{-2 / 3}$.

\subsection{Late-time solution}

We consider the late-time solution of the nonlinear equation (6.74) in terms of the new variable

$$
T=\mu \varepsilon t
$$

We can rewrite the equation in terms of the new variable $T$ by representing the time derivatives as

$$
\frac{\partial}{\partial t}=\mu \varepsilon \frac{\partial}{\partial T}
$$

Equation (6.74) becomes

$$
\begin{array}{r}
\left(\mu \varepsilon \frac{\partial}{\partial T}+\mu \bar{u} \frac{\partial}{\partial X}\right)^{2} \psi_{z z}+O\left(\delta \mu^{2}\right)+\mu^{2} N^{2} \psi_{X X}= \\
-\varepsilon\left(\varepsilon \mu \frac{\partial}{\partial T}+\mu \bar{u} \frac{\partial}{\partial X}\right)\left(\mu \psi_{X} \psi_{z z z}-\mu \psi_{z} \psi_{z z X}\right)+O\left(\varepsilon \mu^{2} \delta\right)
\end{array}
$$

If we divide this equation by $\mu^{2}$ we see that this equation does not depend on $\mu$ :

$$
\left(\varepsilon \frac{\partial}{\partial T}+\bar{u} \frac{\partial}{\partial X}\right)^{2} \psi_{z z}+N^{2} \psi_{X X}=-\varepsilon\left(\varepsilon \frac{\partial}{\partial T}+\bar{u} \frac{\partial}{\partial X}\right)\left(\psi_{X} \psi_{z z z}-\psi_{z} \psi_{z z X}\right)+O(\varepsilon \delta)
$$


So, the solution of the equation depends only on the parameter $\varepsilon$. We consider two cases: $\varepsilon \sim 1$ and $\varepsilon \ll 1$.

In the first case, when $\varepsilon \sim 1$, the governing equation is

$$
\left(\frac{\partial}{\partial T}+\bar{u} \frac{\partial}{\partial X}\right)^{2} \psi_{z z}+O(\delta)+N^{2} \psi_{X X}=-\left(\frac{\partial}{\partial T}+\bar{u} \frac{\partial}{\partial X}\right)\left(\psi_{X} \psi_{z z z}-\psi_{z} \psi_{z z}\right)+O(\delta)
$$

This equation is strongly nonlinear, and for this case we cannot find an analytical solution using asymptotic methods. As will be shown in the next chapter, the numerical solution also does not converge for large values of $\varepsilon$.

For the case where $\varepsilon \ll 1$ we look for a solution in powers of $\varepsilon$

$$
\psi=\psi^{(0)}+\varepsilon \psi^{(1)}+\varepsilon^{2} \psi^{(2)}+O\left(\varepsilon^{3}\right) .
$$

Substituting this series into the governing nonlinear equation we get

$$
\begin{aligned}
& O(1): \bar{u}^{2} \psi_{X X z}^{(0)}+N^{2} \psi_{X X}^{(0)}=0 \\
& O(\varepsilon): 2 \bar{u} \psi_{z z X T}^{(0)}+\bar{u}^{2} \psi_{X}^{(1)} X z z+N^{2} \psi_{X X}^{(1)}=-\bar{u}\left(\psi_{X}^{(0)} \psi_{z z z}^{(0)}-\psi_{z}^{(0)} \psi_{z z X}^{(0)}\right)
\end{aligned}
$$

The solution for these equations could be possibly found by asymptotic methods, but also by numerical simulation. In the next chapter numerical simulations for the nonlinear case are presented and then in the final chapter we present a discussion and summary of our results. In the next chapter the numerical simulation for the nonlinear case will be discussed. 


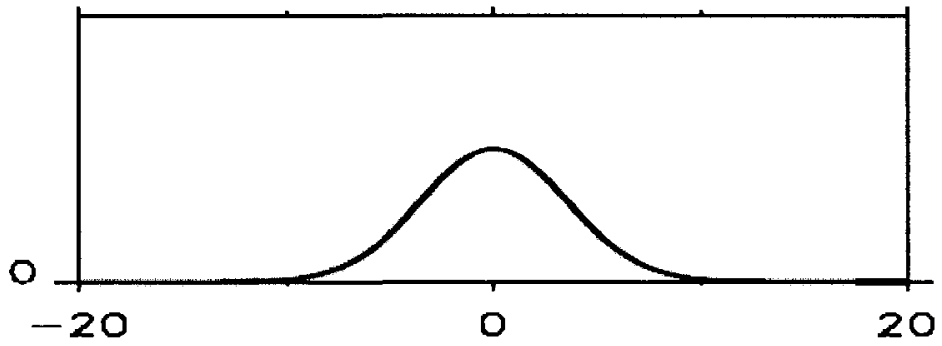

Figure 6.1: Boundary conditions in the form of an isolated mountain $\psi=e^{-\mu^{2} x^{2}}$ with length $\sim \mu^{-1}$. 


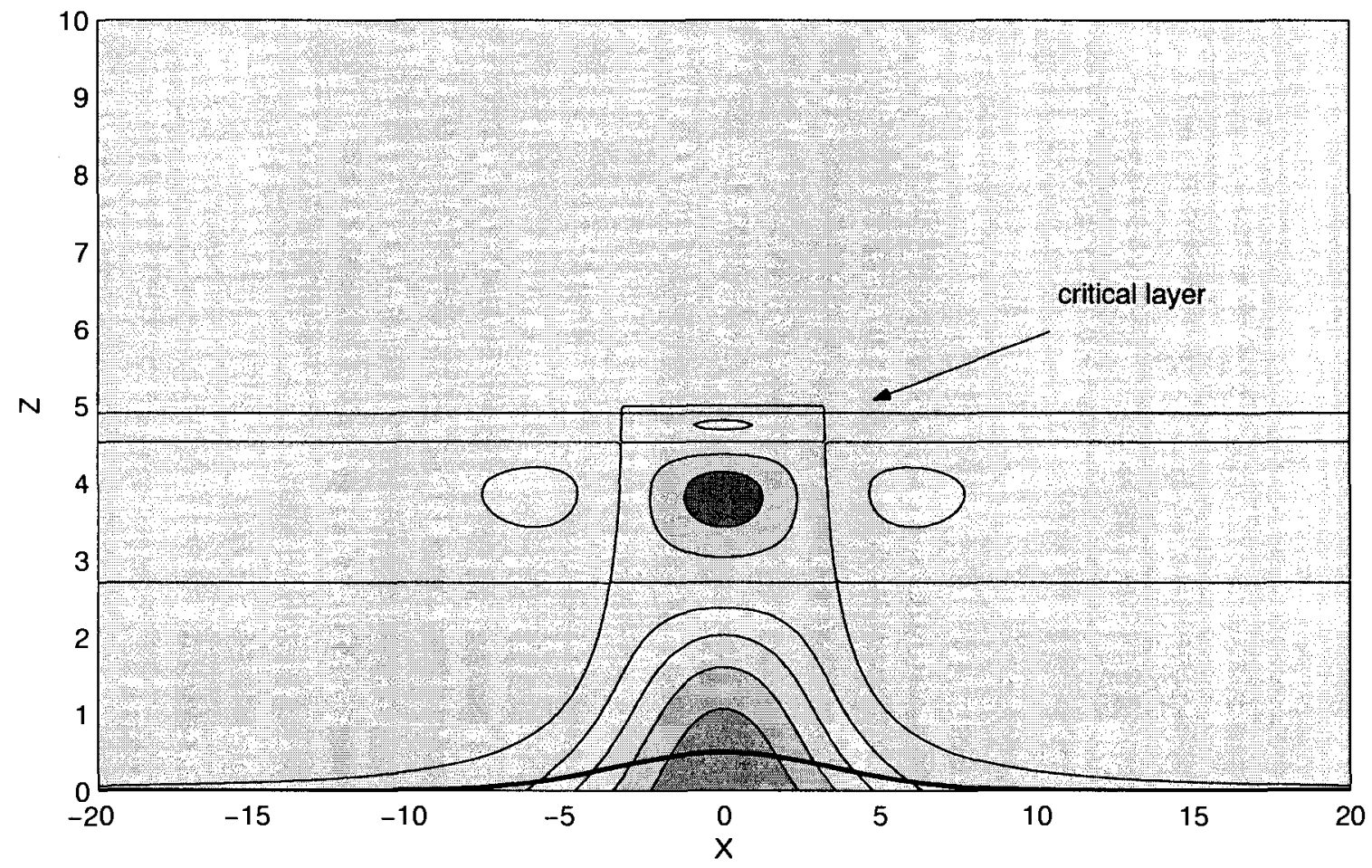

Figure 6.2: Contours of the streamfunction $\psi(X, z)(6.39)$ with the boundary condition $A(\mu x)=e^{-\mu^{2} x^{2}}, N=2.0, \mu=0.2$ and $\delta=0.1$. 


\section{Chapter 7}

\section{Numerical study of gravity waves over an isolated topography}

The goal of the numerical simulations is to continue the analytic study into the nonlinear regime $(\varepsilon \neq 0)$ for which analytic solutions are hard to derive. The numerical methods used in this chapter were developed for study a nonlinear critical layer evolution of a forced Rossby wave packet (Campbell and Maslowe, 2001) and for the nonlinear critical-layer evolution of a forced gravity wave packet (Campbell and Maslowe, 2003).

\subsection{Description of the numerical methods}

In this simulation the wave packet, localized in the horizontal direction, is forced at the lower boundary of a two-dimensional domain and propagates vertically towards the critical layer. The numerical simulations are carried out on a rectangular domain defined by $x_{1} \leq x \leq x_{2}$ and $z_{1} \leq z \leq z_{2}$. The forcing is applied at the lower boundary, 
$z_{1}=0$, and is of the form

$$
\psi=A(x) e^{i k x} f(t)+c . c .
$$

where

$$
f(t)= \begin{cases}t / t_{1}, & \text { if } t<t_{1} \\ 1, & \text { otherwise }\end{cases}
$$

With this forcing function, the amplitude of the disturbance at $z_{1}$ is increased with time from zero at $t=0$ and then kept fixed after a certain time $t=t_{1}$. The reason for this delay of forcing was to prevent instabilities from developing near the inflow boundary, which could occur if the time step used in the numerical solution was not sufficiently small. The initial condition is that everything is zero for $t \leq 0$ until the forcing is switched on at $t=0$.

For our problem where we consider an isolated topography, we set $k=0$ and $A(x)=e^{-\mu^{2} x^{2}}$ with $\mu$ constant. The initial velocity and density profile were taken to be $\bar{u}=\tanh \alpha_{0}\left(z-z_{c}\right)$ and $\bar{\rho}=\rho_{0} e^{-z / H}$, where $\alpha_{0}, \rho_{0}, H$ and $z_{c}$ are non-dimensional constants and $z_{c}$, the location of the critical level, was set to 5 . With this choice of $\bar{\rho}$ the Brunt-Väisälä frequency is given by

$$
N^{2}=-\frac{g}{\bar{\rho}} \frac{d \bar{\rho}}{d z}=\frac{g}{H}
$$

where $H$ is the scale height. So $N$ is constant, as was assumed in the analytic solution. At the upper boundary $z=10$ everything is kept fixed at zero. This is a reasonable boundary condition to use since in most cases the disturbances do not get through the critical level. The horizontal extent of the domain is chosen to be large enough to ensure that the amplitude of the disturbance would decay to zero at the boundaries as required in the analytic solution. 


\section{Horizontal discretization}

The system of equations (3.21) and (3.27) for the vorticity $\zeta$ and the density $\rho$

$$
\begin{gathered}
\zeta_{t}+\bar{u} \zeta_{x}-\bar{u}^{\prime \prime} \psi_{x}+g(\bar{\rho})^{-1} \rho_{x}+\varepsilon\left(\psi_{x} \zeta_{z}-\psi_{z} \zeta_{x}\right)-\operatorname{Re}^{-1} \nabla^{2} \zeta+R e^{-1} \varepsilon^{-1} \bar{u}^{\prime \prime \prime}=0 \\
\rho_{t}+\bar{u} \rho_{x}+\bar{\rho}^{\prime} \psi_{x}+\varepsilon\left(\psi_{x} \rho_{z}-\psi_{z} \rho_{x}\right)-\operatorname{Re}^{-1} \operatorname{Pr}^{-1} \nabla^{2} \rho-\operatorname{Re}^{-1} \operatorname{Pr}^{-1} \varepsilon^{-1} \bar{\rho}^{\prime \prime}=0
\end{gathered}
$$

and

$$
\zeta=\nabla^{2} \psi
$$

were solved numerically using Fourier transform methods for the $x$ discretization. We define the Fourier transform by

$$
\begin{gathered}
\tilde{\psi}(\lambda, z, t)=\int_{-\infty}^{\infty} \psi(x, z, t) e^{-i \lambda x} d x=\mathcal{F}(\psi(x, z, t)) \\
\psi(x, z, t)=\frac{1}{2 \pi} \int_{-\infty}^{\infty} \tilde{\psi}(\lambda, z, t) e^{i \lambda x} d \lambda
\end{gathered}
$$

The transformed equations

$$
\tilde{\zeta}_{t}+i \lambda\left(\bar{u} \tilde{\zeta}-\bar{u}^{\prime \prime} \tilde{\psi}+g(\bar{\rho})^{-1} \tilde{\rho}\right)+\varepsilon \mathcal{F}\left(\psi_{x} \zeta_{z}-\psi_{z} \zeta_{x}\right)-R e^{-1}\left(\tilde{\zeta}_{z z}-\delta \kappa^{2} \tilde{\zeta}\right)+R e^{-1} \varepsilon^{-1} \bar{u}^{\prime \prime \prime}=0
$$

$$
\begin{gathered}
\tilde{\rho}_{t}+i \lambda\left(\bar{u} \tilde{\rho}+\bar{\rho}^{\prime} \tilde{\psi}\right)+\varepsilon \mathcal{F}\left(\psi_{x} \rho_{z}-\psi_{z} \rho_{x}\right)-\operatorname{Re}^{-1} \operatorname{Pr}^{-1}\left(\tilde{\rho}_{z z}-\delta \lambda^{2} \tilde{\rho}\right)+\operatorname{Re}^{-1} \operatorname{Pr}^{-1} \varepsilon^{-1} \rho u^{\prime \prime \prime}=0 \\
\tilde{\zeta}=\tilde{\psi}_{z z}-\delta \lambda^{2} \tilde{\psi}
\end{gathered}
$$

are solved numerically. The nonlinear terms are calculated using a pseudo-spectral method, with the Fourier integrals evaluated by the trapezoidal approximation. With 
this method, an integral

$$
\tilde{\psi}(\lambda)=\int_{-\infty}^{\infty} \psi(x) e^{-i \lambda x} d x
$$

would be approximated by a series calculated at a set of points $x_{l}=x_{0}+l \Delta x$, $l=0,1, \ldots, N$, for wave numbers $\lambda_{m}=\lambda_{0}+m \Delta \lambda, m=0,1, \ldots, N$. This is a valid approximation provided $\lambda$ is not too large. The wave number domain is $\lambda_{1}<\lambda<\lambda_{2}$ where $\lambda_{1}$ and $\lambda_{2}$ are chosen so that the wave number interval is wide enough to accommodate the higher wave numbers that arise in the nonlinear computations, e.g., in the graph shown here (Figure 8.1), we have $-20<\lambda<20$. For linear computations we only need two wavenumbers $\lambda=k$ and $\lambda=-k$.

Denoting the approximations for $\tilde{\psi}\left(\lambda_{m}\right)$ and $\psi\left(x_{l}\right)$ by $\tilde{\psi}_{m}$ and $\psi_{l}$ respectively, we can write

$$
\tilde{\psi}_{m}=\Delta x \sum_{l=0}^{N-1} \psi_{l} e^{-i \lambda_{m} x_{l}}, \quad-\infty<\lambda<\infty
$$

or

$$
\tilde{\psi}_{m}=\Delta x e^{-i \lambda_{m} x_{0}} \sum_{l=0}^{N-1} \psi_{l} e^{-i l m \lambda_{0} \Delta x} e^{-i l m \Delta x \Delta \lambda} .
$$

If the grids of the $x$ and $\lambda$ values are chosen in such a way that

$$
(\Delta x)(\Delta \lambda)=2 \pi / N
$$

then the series takes the form

$$
\tilde{\psi}_{m}=\Delta x e^{-i \lambda_{m} x_{0}} \sum_{l=0}^{N-1} \psi_{l} e^{-i l \lambda_{0} \Delta x} e^{-2 \pi i l m / N}
$$

and can be evaluated in $O(N \log N)$ operations using the Fast Fourier Transform 
(FFT) algorithm, if $N$ is chosen to be a power of 2 . In general if

$$
(\Delta x)(\Delta \lambda) \neq 2 \pi / N
$$

then a generalization of the FFT called the Fractional Fourier Transform (FRFT) can be used (Bailey and Swarztrauber, 1991, 1994). This technique is implemented in the numerical code.

\section{Vertical discretization}

In the $z$ direction a finite difference approximation was used to evaluate the $z$ derivatives. The numerical code uses two different methods. One version of the code uses regular centred differences, i.e. for the first derivative of a function $\phi(z)$

$$
\phi_{j}^{\prime} \sim \frac{\phi_{j+1}-\phi_{j-1}}{2 \Delta z}
$$

where $\phi_{j}$ represents the approximation to $\phi$ at $z_{j}$ for $j=0,1, \ldots, J$ on a grid of points $z_{j}=z_{1}+j \Delta z$, with $\Delta z=\left(z_{2}-z_{1}\right) / J$. And for the second derivative,

$$
\phi_{j}^{\prime \prime} \sim \frac{\phi_{j+1}-2 \phi_{j}+\phi_{j-1}}{2 \Delta z^{2}}
$$

The other version of the code uses high-order compact finite difference approximation. These methods have a number of advantages over other methods: they give a better representation of short length scales than traditional finite difference schemes. A finite difference scheme is compact if the approximations for the derivatives of a function at a given point on the mesh involve only adjacent points. In high order compact schemes, an approximation for a derivative is evaluated by solving a system of equations. For example, on a mesh $z_{j}, j=0,1, \ldots, N$ with uniform step size $\Delta z$, an approximation 
$\phi_{j}^{\prime}$ for the first derivative of a function $\phi(z)$ can be defined by the tridiagonal system

$$
\frac{1}{6}\left(\phi_{j+1}^{\prime}+4 \phi_{j}^{\prime}+\phi_{j-1}^{\prime}\right)=\frac{\phi_{j+1}-\phi_{j-1}}{2 \Delta z}
$$

By expanding all the terms in the approximation in a Taylor series about the point $z_{j}$, it can be shown that the truncation error of the approximation is $\left(\Delta y^{4} / 180\right) \phi_{j}^{V}$, since the terms up to order $O\left(\Delta z^{3}\right)$ cancel out. Thus, fourth order accuracy is achieved while maintaining the compactness of the scheme. Similarly, a fourth order compact approximation for the second derivative is given by

$$
\frac{1}{12}\left(\phi_{j+1}^{\prime \prime}+10 \phi_{j}^{\prime \prime}+\phi_{j-1}^{\prime \prime}\right)=\frac{\phi_{j+1}-2 \phi_{j}+\phi_{j-1}}{\Delta z^{2}}
$$

The advantage of using this method is improved accuracy. The disadvantage is that it is more expensive. In this study both methods were used. The results shown here are for simulations with regular centred differences.

\section{Non-uniform mesh for the critical layer}

In the critical layer and above, finer resolution was required than in the region below, where there was little or no wave activity. Therefore, a mesh with non-uniform spacing was needed. But evaluating derivatives on a non-uniform mesh would reduce the accuracy of the scheme, since then the terms up to order $O\left(\Delta z^{3}\right)$ would then not cancel out in the Taylor expansion for the approximation.

To evaluate the first and second derivatives of a function on a non-uniform mesh using compact finite differences, we first construct a uniform mesh of points, $Z_{j}=$ $j \Delta z$, and then define a non-uniform grid $z_{j}$ by $z=F(Z)$, where $F$ is some twice differentiable function that maps the set of points $Z_{j}$ onto the set $z_{j}$. Derivatives with 
respect to $Z$ are transformed into $z$-derivatives using the chain rule

$$
\frac{\partial}{\partial z}=\frac{\partial}{\partial Z} \frac{\partial Z}{\partial z}, \quad \frac{\partial^{2}}{\partial z^{2}}=\frac{\partial^{2}}{\partial Z^{2}}\left(\frac{\partial Z}{\partial z}\right)^{2}+\frac{\partial}{\partial Z} \frac{\partial^{2} Z}{\partial z^{2}}
$$

We define the mapping $F$ in such a way as to double the resolution in the critical layer region and to halve it in the region above the critical layer, but keep it unchanged below the critical layer. In order to satisfy these requirements exactly, we would need to define $z$ in terms of $Z$ by a piecewise linear function of the form

$$
z=\left\{\begin{array}{lll}
Z, & \text { if } & 0 \leq Z \leq 4 \\
Z / 2+2 & \text { if } & 4 \leq Z \leq 8 \\
2 Z-10, & \text { if } & 8 \leq Z \leq 10
\end{array}\right.
$$

But, clearly, this function is not differentiable at $Z=4$ and 8 . It is necessary, therefore, to define the mapping by constructing a differentiable function that would approximate the above function in the interval $0 \leq Z \leq 10$. This function is

$$
z=Z+\frac{Z^{2}(Z-4)(Z-10)}{288}
$$

which is continuous and twice differentiable for all real $Z$, and quite closely approximates the function (7.22). By experimenting with the mesh spacing, reducing it until the computed solution was found to be independent of the mesh size, it was found that a minimum of 20 points was needed in the critical layer to represent the evolution of the disturbance accurately. The time-derivatives were discretized for using the second order Adams-Bashforth method and the time-step size set to 0.02 non-dimensional units, a value small enough to ensure stability of the computations. 


\subsection{Solutions for $k \neq 0$ (Campbell and Maslowe, 2003)}

Campbell and Maslowe (2003) did a standard run with the following input parameters $k=2, \mu=0.2, \delta=0.2, N=2.0$ for the wave-packet forcing. Some of their results are shown in Figures $7.1-7.3$ which correspond to Figures 2, 3, and 5 in their paper (Campbell and Maslowe, 2003). In Figure 7.1 the horizontal velocity perturbation $u(x, z, t)=-\psi_{z}$ at early time $(t=10)$ is plotted. It is seen that there is almost complete net absorption of the disturbance at the critical level $\left(z_{c}=5\right)$. To see the behaviour of the solution across the critical layer, the vertical momentum flux $F$ (see Appendix D for the definition and detailed description of the momentum flux) was plotted in Figure 7.2. The flux was seen to be discontinuous across the critical layer, negative below and zero above. This fact proves the assumption that the gravity waves are almost absorbed at the critical layer at least at the early time.

The jump in the momentum flux across the critical level varies in time. In Figure 7.3 the jump $[F]$ across the critical layer was calculated and plotted as a function of time. In the linear case, when $\varepsilon$ is set to zero, the jump increases initially during the switch-on time, but in this short time attains a steady state. This is shown by the dashed line. The solid line corresponds to the nonlinear case, when $\varepsilon=0.05$. In this case $[F]$ is increasing at early time, however, no steady state is attained, the nonlinear effects soon become evident, and $[F]$ starts to decrease rapidly and goes to zero which indicates wave reflections as discussed in Appendix D. 


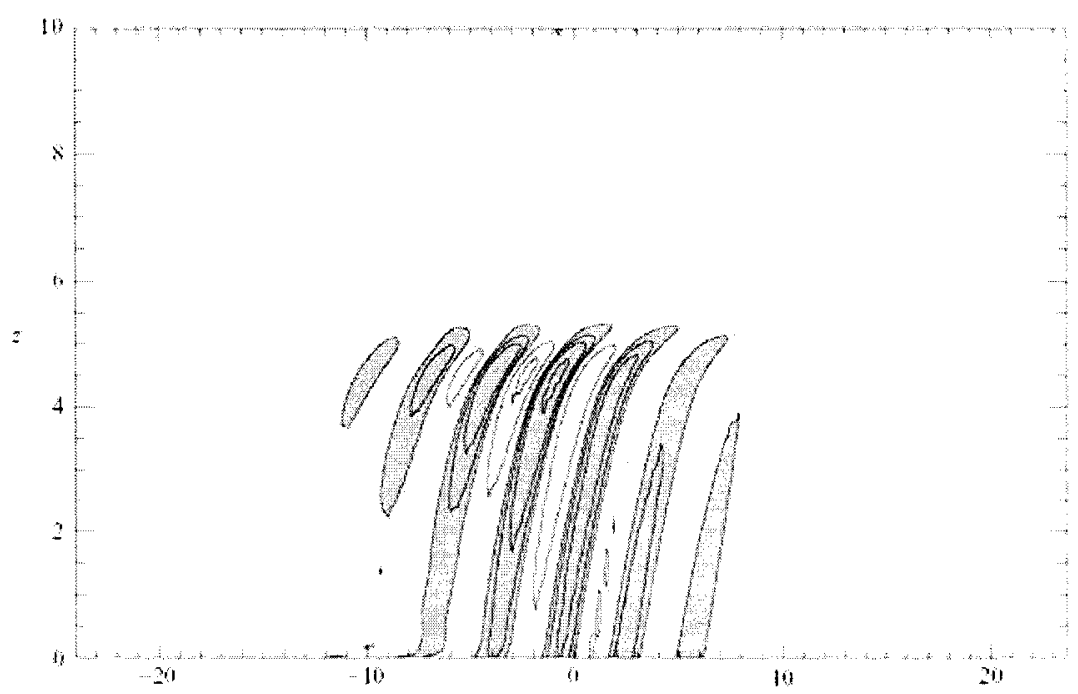

Figure 7.1: Wave packet forcing: $e^{-\mu^{2} x^{2}} e^{2 i x}$. Horizontal velocity perturbation as a function of $x$ and $z$ at $t=10$. Parameters: $\varepsilon=0.05, \mu=0.2, N=2.0$. Regions where $u>0$ are shaded.

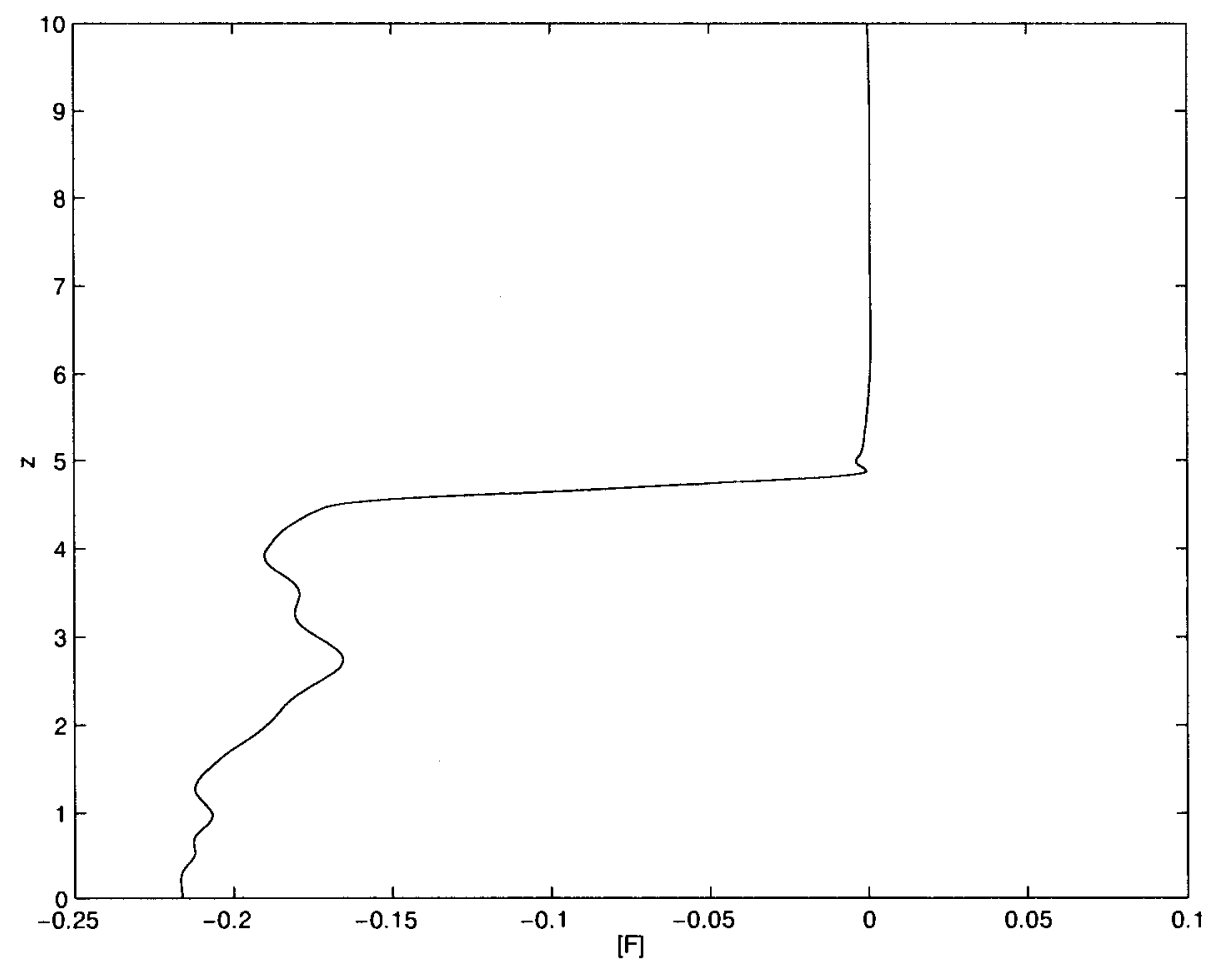

Figure 7.2: Vertical variation of the mean vertical momentum flux $F(z, t)$ for the wave packet forcing $e^{-\mu^{2} x^{2}} e^{2 i x}$. The result obtained at $t=50$. Parameters: $\varepsilon=0.05$, $\mu=0.2, N=2.0$. 


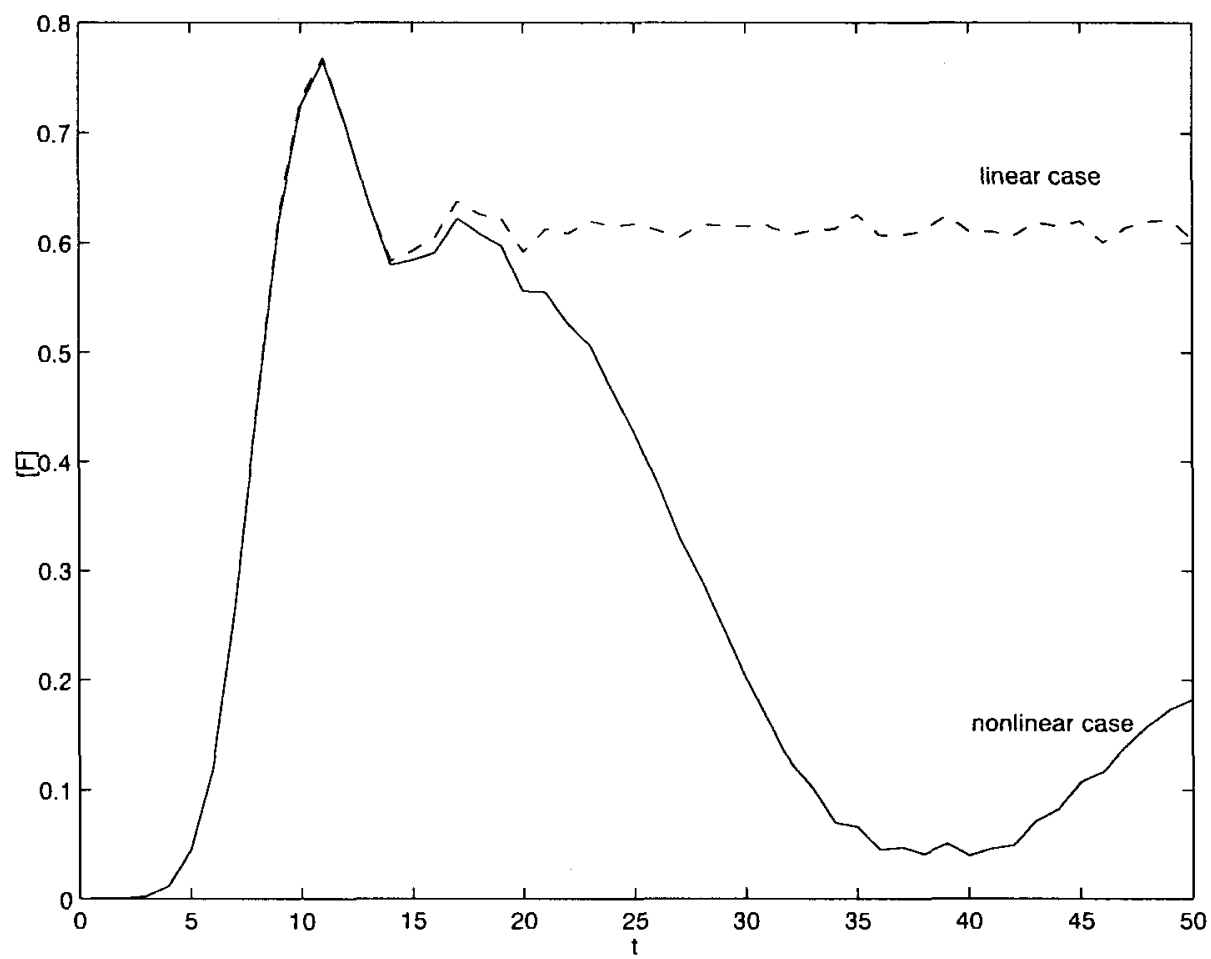

Figure 7.3: Variation of the jump in the momentum flux with $t$. Comparison of the results obtained with the wave-packet forcing $e^{-\mu^{2} x^{2}} e^{2 i x}$ for the linear case $\varepsilon=0$ (dashed line) and for the nonlinear case $\varepsilon=0.05$ (solid line). $\mu=0.2, N=2.0$. 


\subsection{Results of the numerical simulation for the grav- ity wave over an isolated mountain}

In our simulation we set $k=0$. In Figure 7.4 we plotted the streamfunction of the gravity waves forced by an isolated mountain with the boundary condition $\psi\left(X, z_{1}, t\right)=$ $e^{-\mu^{2} x^{2}}$. The streamfunction is plotted for different moments of time: $t=2, t=5$, $t=10$, and $t=40$ for the nonlinear case when $\varepsilon=0.2$. We can conclude that initially gravity waves propagate upward, but soon they are absorbed by the critical layer, and at later time (here it is $t=10$ ) the gravity waves exist only under the critical level $\left(z=z_{c}\right)$. Note, that Figure 7.4 (d) looks approximately like Figure 6.2, where the analytical solution for the steady case was plotted. It supports the fact that when $t \rightarrow \infty$ the time-dependent solution is converging to the steady solution.

Absorption of the gravity waves by the critical layer is shown also in Figure 7.5, where the variation of the momentum flux with height is plotted. As in the previous study with the wave packet forcing (see section 7.2), in the case of an isolated mountain the momentum flux changes from negative under the critical level to zero above the critical level. In the linear case with $\varepsilon=0$ the graph (dashed line) looks like a footstep because of the Elliassen-Palm theorem (see Appendix D) which states that the momentum flux is independent of height everywhere where the flow is nonsingular. We see that in the nonlinear case, when $\varepsilon=0.2$ (solid line), the momentum flux changes smoothly and the absolute value of the momentum flux is less than in the linear case. This means that the nonlinear terms restraints the flow. This conclusion will be supported also by Figure 7.6.

To investigate the nonlinear problem we want to examine three cases which were discussed in section 6.4 for the nonlinear inner solution. We discussed three cases with different relations between the small parameters $\mu$ and $\varepsilon$. We will consider the 

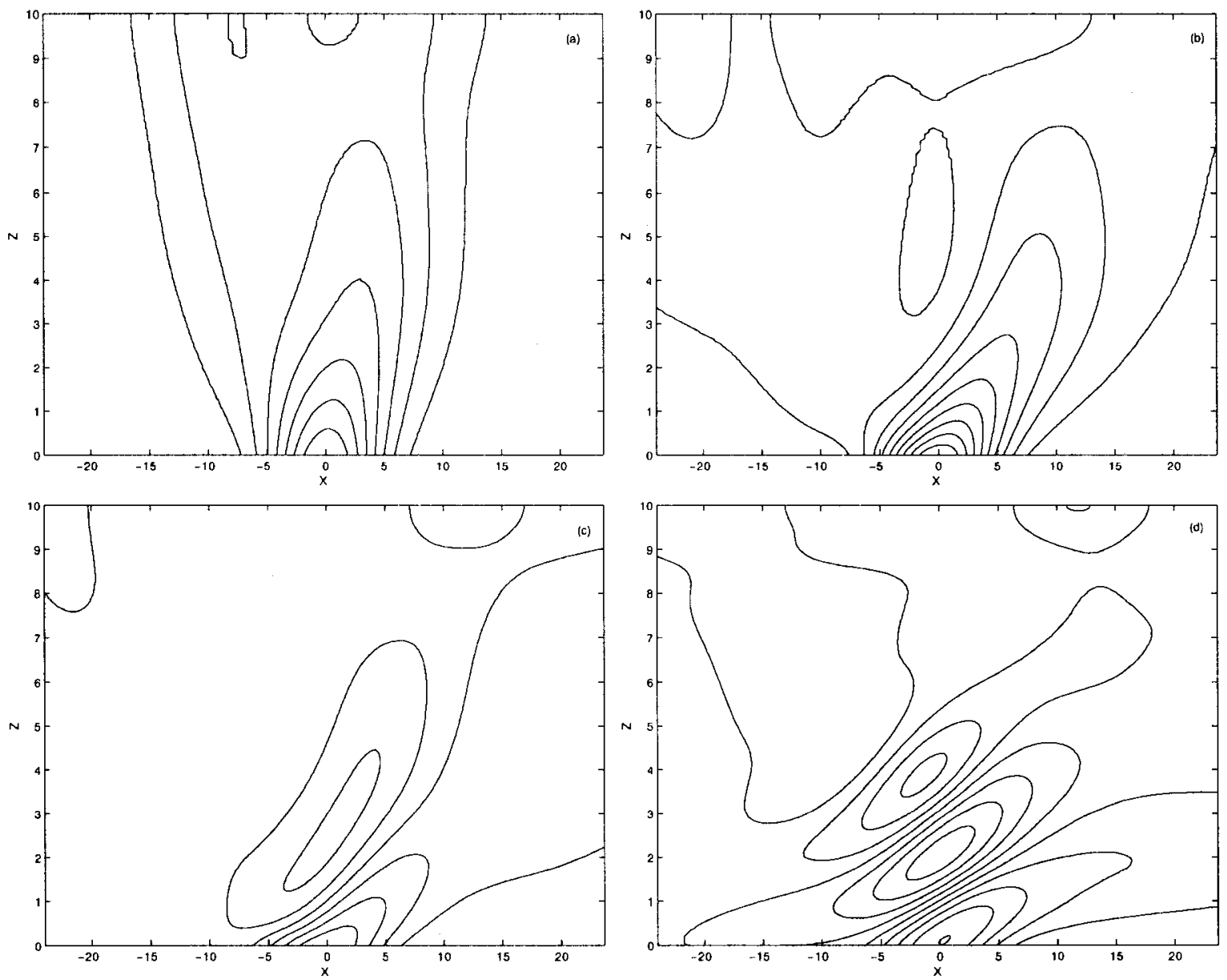

Figure 7.4: Developing of the gravity wave packet forced by isolated mountain $e^{-\mu^{2} x^{2}}$. Contour plots of streamfunction $\psi(X, z, t)$ obtained at $t=2(\mathrm{a}), t=5(\mathrm{~b}), t=10$ (c),$t=40$ (d) for the nonlinear case when $\varepsilon=0.2$. Parameters: $\mu=0.2, N=2.0$. 


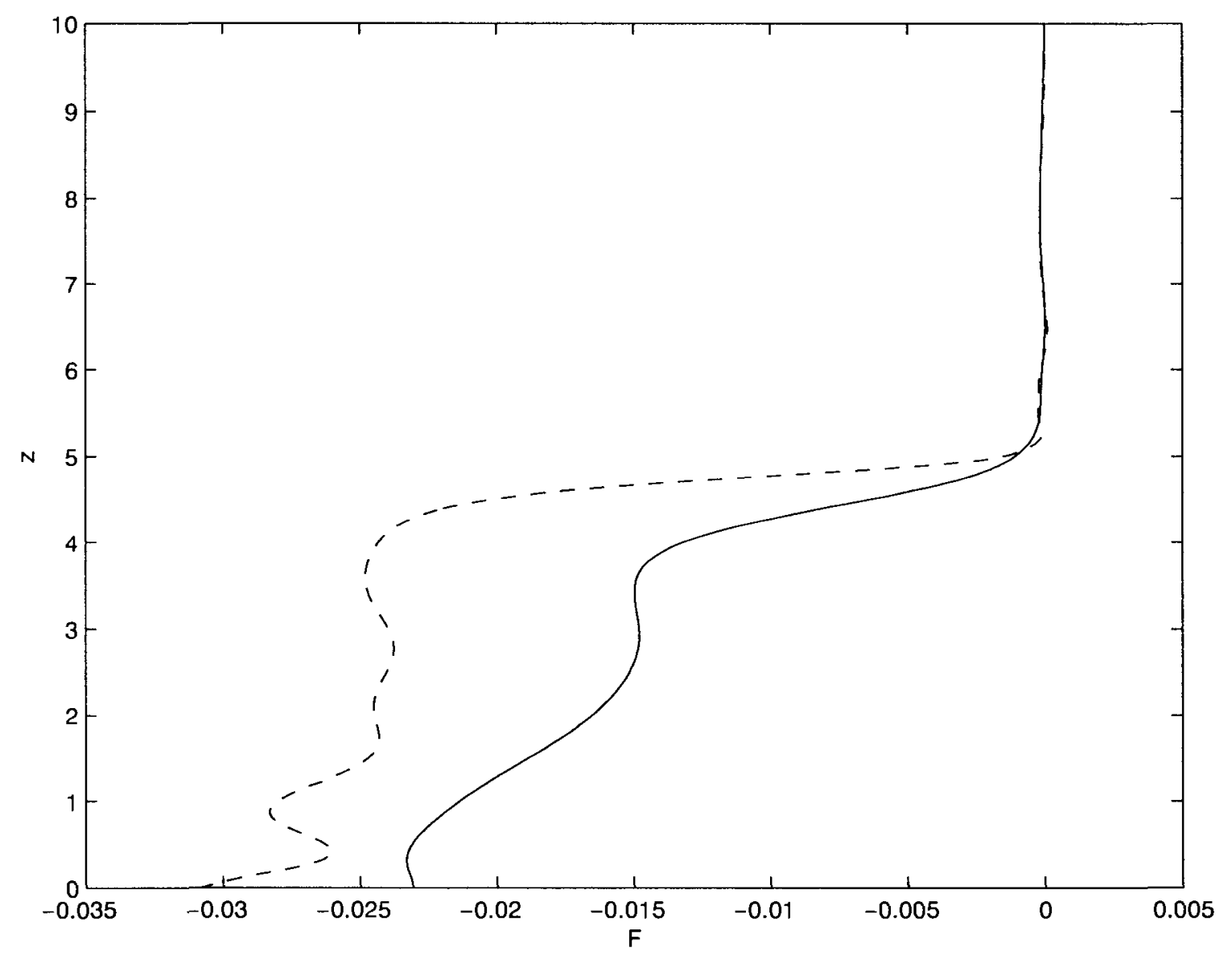

Figure 7.5: Momentum flux as a function of z. $t=10$. Gravity waves forced by an isolated mountain $e^{-\mu^{2} x^{2}}$. Parameters: $\mu=0.2, N=2.0, t=50.0$. the momentum flux is plotted for the nonlinear case (solid line) where $\varepsilon=0.2$ and for the linear case where $\varepsilon=0$ (dashed line). 
cases

- $\varepsilon \ll \mu$,

- $\varepsilon=\mu$,

- $\varepsilon>>\mu$.

We will keep $\mu$ fixed $\mu=0.2$. For the case $\varepsilon>>\mu$ we tried $\varepsilon>0.2$. However, the solution is unstable for any $\varepsilon>0.2$. There is a large amount of wave reflection at the critical layer and transmission through the critical level, and the solution very quickly becomes unstable. To carry out a nonlinear computation with such a large value of $\varepsilon$, we would need to find a way to deal with wave reflections at the upper and lower boundaries. In Figure 7.6 we compare two plots for the streamfunction $\psi(x, z, t)$ for the cases $\varepsilon=0.01 \ll \mu$ and $\varepsilon=0.2=\mu$. The streamfunctions are plotted for time $t=50$. At the early time the difference between these two cases is negligible. At $t=50$ it is evident that when $\varepsilon$ is very small and the solution is close to the linear case, the gravity wave packet spreads in the horizontal direction more than when $\varepsilon=0.2=\mu$. So we can conclude that the nonlinear terms restrain the horizontal spreading of the wave packet. This conclusion is in agreement with the paper of Campbell and Maslowe (2003). 

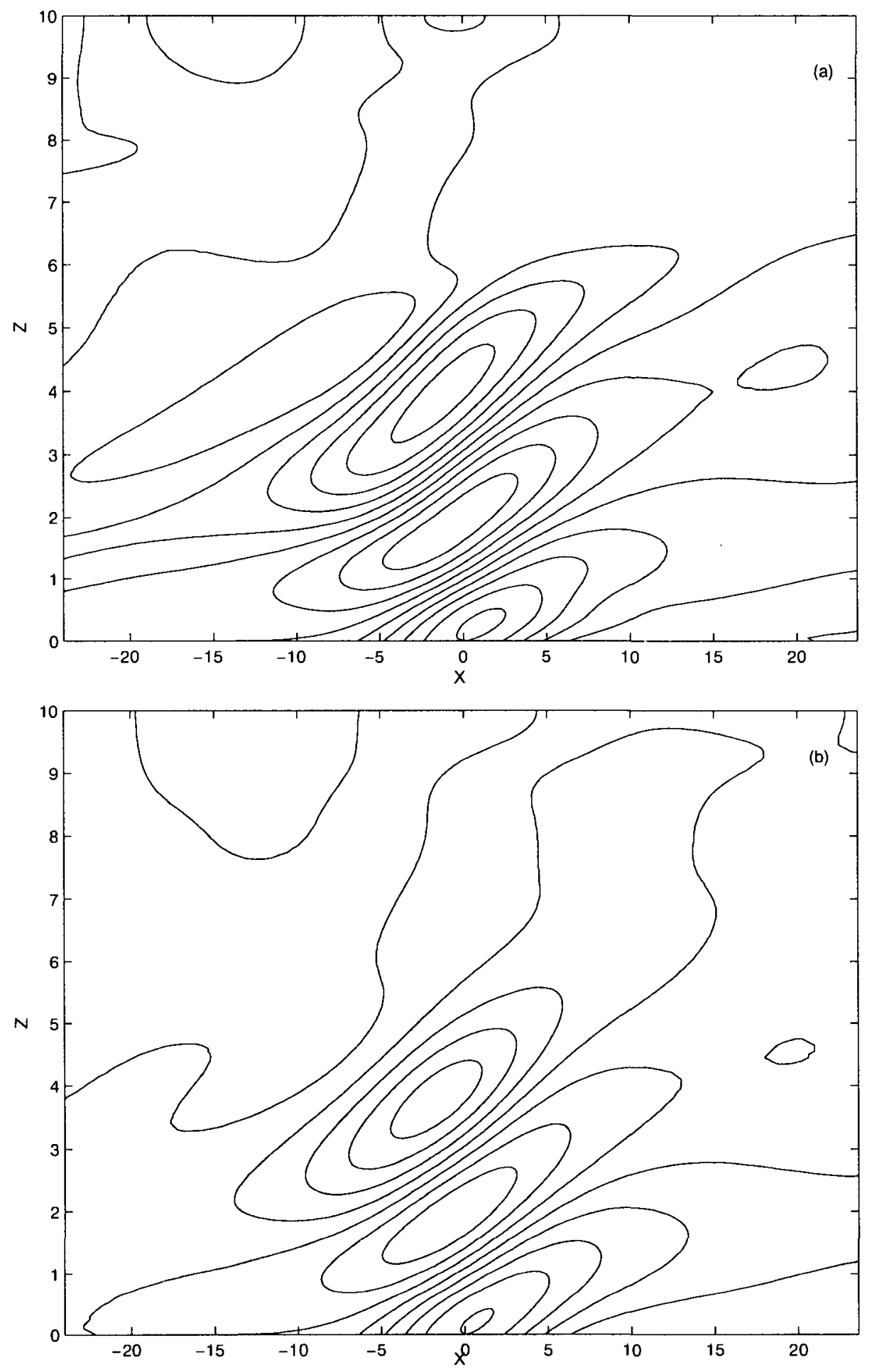

Figure 7.6: Gravity wave packet forced by an isolated mountain $e^{-\mu^{2} x^{2}}$. Contour plots of streamfunction $\psi(X, z, t)$ for the case $\varepsilon=0.01$ (a) and $\varepsilon=0.2$ (b) The results are obtained at $t=50$. Parameters: $\mu=0.2, N=2.0$. 


\section{Chapter 8}

\section{Conclusions}

In Chapters 1-4 we derived the governing equations for our study and presented some basic background information before proceeding to derive analytical solutions for various cases. Chapter 5 described the time-dependent problem in which the waves are assumed to be periodic in the horizontal direction only, but the wave amplitude varies in time and in the vertical direction. The governing time-dependent equations were linearized and approximate analytical solutions were derived for the two cases: a wave forced by a monochromatic horizontally-periodic boundary condition $e^{i k x}$, and a wave packet forced by a horizontally-localized boundary condition like a mountain range $A(\mu x) e^{i k x}$. In the monochromatic case the solution was first derived by Booker and Bretherton (1967) and we described it here. For the wave packet case numerical solutions for the linear and nonlinear problem were obtained by Campbell and Maslowe (2003). In this thesis an approximate analytic solution for the linear case was derived using Booker and Bretherton's (1967) monochromatic solution as a starting point. In Chapter 6 we derived analytic solutions for the case where $k=0$. In Chapter 7 we described the numerical solution of the problem. 


\subsection{Discussion of the analytical solutions}

In Chapter 6 we considered the solution of the problem of the gravity waves forced by isolated topography. We obtained solutions both for the steady case and the time-dependent case. We included into our consideration three small parameters $\mu$, $\varepsilon$ and $\delta$. Here $\mu$ determines the width of the mountain, $\varepsilon$ determines the magnitude of perturbation of the wave amplitude because of the mountain height, and $\delta$ is the aspect ratio which determines the relative magnitude of horizontal and vertical scales in the problem. We solved the governing equation by expanding our solutions in series of powers of these small parameters.

For the steady case we considered two situations, when the mean flow $\bar{u}$ is constant and when the mean flow is a linear function of $z$. For constant $\bar{u}$ the solution presents gravity waves with a vertical wavenumber of $\frac{N}{\bar{u}}$. The wavenumber and amplitude of the wave do not depend on the height. This solution has no singularities.

For the second case, when the mean flow is a linear function of $z$, the solution is singular at the critical level where the phase speed of the gravity waves equals to the mean flow velocity. The solution consists of a leading term which corresponds to the solution of the steady Taylor-Goldstein equation and additional terms of smaller orders in powers of $\delta \mu^{2}$. The solution has a singularity at the critical level, and the wavenumber of the gravity wave packet decreases with height. For long waves $(\delta=0)$ the wave packet maintains the horizontal profile that is specified at the lower boundary. With a nonzero aspect ratio $\delta$ the horizontal shape of the wave packet changes with height as shown in Figure (6.2).

To derive a time-dependent solution we also expand the solution in a series of powers of small parameters. The leading term of the solution is the sum of the steady solution, the same as before, and a time-dependent part. The time-dependent term is 
proportional to the height over the mountain and decreases with time. So, as $t \rightarrow \infty$ the leading term corresponds to the steady solution.

To find the exact expression for the time-dependent term we had to consider the so-called inner layer - the thin layer in the vicinity of the critical level, where the obtained solution is singular. When we derived the solution in the inner layer we had two aims: to find the time-dependent term in the outer solution and to avoid the singularity of the solution inside the inner layer. We obtained a non-singular solution inside the inner layer. Matching the two solutions at the boundary between the inner layer and the outer region we obtained an expression for the time-dependent term of the outer solution.

Up to that point we had solved the problem in the linear approximation. But in the linear problem the critical layer thickness decreases with time as $t^{-1}$. This means that we have to consider the non-linear problem to get a solution for the inner layer. The nonlinear time-dependent solution for the inner layer is derived. However, the solution grows with time and to find the inner layer solution properly we have to consider a "late-time" solution. The differential equations for the late-time nonlinear solution were derived.

\subsection{Discussion of the numerical solutions}

For the analytical study in Chapter 6 it became clear that in order to proceed any further with the nonlinear solutions we would have to use numerical rather than analytical techniques. Thus in Chapter 7 we made use of the numerical code developed by Campbell and Maslowe (2003) and adapted it to suit our problem. With $k=0$ we saw that the wave packet was absorbed at the critical layer at early time and there was a jump in the momentum flux at the critical level, as expected from the analytic 
solutions. This is similar to the $k \neq 0$ case. We carried out nonlinear solutions with $\varepsilon=0.01<\mu$ and $\varepsilon=0.2=\mu$ to be able to compare the different cases discussed in section 6.4. The nonlinearity restricts the horizontal spreading out of wave packet. We saw that with larger $\varepsilon$ the restriction is stronger than in the case where $\varepsilon$ is smaller. A possible explanation for this is that in the case of small $\varepsilon$ the wave packet is absorbed at the critical level and there is an outward horizontal momentum flux near the critical level which allows the absorption of packet to continue to late time. When $\varepsilon$ is large, however, there is less absorption and there is no momentum flux in the horizontal direction so the wave packet remains more confined in the horizontal direction. This explanation has been suggested by earlier researchers Bacmeister and Pierrehumbert (1988). We plan to explore and attempt to understand this better by carrying out further numerical investigations. 


\section{Appendix A}

\section{Laplace transform}

Here we show how to use a Laplace transform to get a solution for section 5.1 . Let $\tilde{\phi}$ be the Laplace transform of $\phi$

$$
\tilde{\phi}(z, s)=\int_{0}^{\infty} \phi(z, t) e^{-s t} d t .
$$

After taking the Laplace transform of (5.3) we have the equation

$$
\left(s+i k\left(z-z_{c}\right)\right)^{2} \tilde{\phi}_{z z}-\left(N^{2}+\delta\right) k^{2} \tilde{\phi}=0 .
$$

with the boundary condition:

$$
\tilde{\phi}\left(z_{1}, s\right)=\int_{0}^{\infty} \phi\left(z_{1}, t\right) e^{-s t} d t=\frac{1}{s}
$$

as $\phi\left(z_{1}, t\right)=1$. We search for the solution of (A.2) in the form

$$
\tilde{\phi} \sim\left(z-z_{c}-i s / k\right)^{\alpha}
$$


So,

$$
\alpha^{2}(\alpha-1)+\left(N^{2}+\delta\right)=0
$$

and

$$
\alpha_{1,2}=\frac{1}{2} \pm i \sqrt{N^{2}+\delta-\frac{1}{4}}=\frac{1}{2} \pm i \gamma
$$

where $\gamma=\sqrt{N^{2}+\delta-1 / 4}$. We consider only upward propagating waves, so we take only $\alpha=\frac{1}{2}+i \gamma$ into account. Applying the boundary condition we get a solution

$$
\tilde{\phi}(z, s)=\frac{1}{s} \frac{\left(z-z_{c}-i s / k\right)^{1 / 2+i \gamma}}{\left(z_{1}-z_{c}-i s / k\right)^{1 / 2+i \gamma}}
$$

We can find the function $\psi(x, z, t)$ by inverting the Laplace transform.

\section{Inverse Laplace transform}

To find the function $\phi$ we use take the inverse Laplace transform of (A.7) to get

$$
\phi(z)=\frac{1}{2 \pi i} \int_{a-i \infty}^{a+i \infty} \frac{1}{s} \frac{\left(z-z_{c}-i s / k\right)^{1 / 2+i \gamma}}{\left(z_{1}-z_{c}-i s / k\right)^{1 / 2+i \gamma}} e^{s t} d s
$$

where $a$ is a real number chosen so that the contour of integration will lie to the right of any singularities of the integrand. The integrand in (A.8) has a pole at $s=0$ and two branch points at

$$
\begin{gathered}
z-z_{c}=\frac{i s}{k} \Longrightarrow s=-i k\left(z-z_{c}\right), \\
z_{1}-z_{c}=\frac{i s}{k} \Longrightarrow s=-i k\left(z_{1}-z_{c}\right) .
\end{gathered}
$$

The integral is evaluated along the contour in the imaginary plane shown in Figure 
A.1. Making use of Cauchy's theorem it is found to be equal to the sum of the contributions from the three singularities: the pole at $s=0$, and the branch points at the point $P$, where $s=-i k\left(z-z_{c}\right)$, and at $P_{1}$ where $s=-i k\left(z_{1}-z_{c}\right)$.

$$
\frac{1}{2 \pi i} \int_{a-i \infty}^{a+i \infty}=\operatorname{Res}(0)-\frac{1}{2 \pi i}\left(\int_{c_{1}}+\int_{c_{2}}+\int_{c_{3}}+\int_{c_{4}}+\int_{c_{5}}+\int_{c_{6}}+\int_{c_{7}}\right)
$$

Contour $C_{1}$ can be described in polar coordinates $(\rho, \theta)$ as

$$
s=a+R e^{i \theta}, \quad \frac{\pi}{2}<\theta<-\frac{\pi}{2}
$$

So,

$$
\begin{array}{r}
\int_{C_{1}} \frac{1}{s} \frac{\left(z-z_{c}-i s / k\right)^{1 / 2+i \gamma}}{\left(z_{1}-z_{c}-i s / k\right)^{1 / 2+i \gamma}} e^{s t} d s= \\
\int_{\pi / 2}^{-\pi / 2} \frac{e^{\left(a+R e^{i \theta}\right) t}}{a+R e^{i \theta}} \frac{\left(z / R-z_{c} / R-i\left(a / R+e^{i \theta}\right) / k\right)^{1 / 2+i \gamma}}{\left(z_{1} / R-z_{c} / R-i\left(a / R+e^{i \theta}\right) / k\right)^{1 / 2+i \gamma}} i R e^{i \theta} d \theta .
\end{array}
$$

When $R \rightarrow \infty$, the integral on the right hand side of (A.12) has the order of $e^{R e^{i \theta}}$ or $e^{R \cos \theta}$. The integral converges to 0 because $\cos \theta$ is negative when $\frac{\pi}{2}<\theta<-\frac{\pi}{2}$. So, the integral along the contour $C_{1}$ converges to 0 .

$$
\int_{C_{1}}=0
$$

The contours $C_{2}$ and $C_{3}$ are circles surrounding the branch points $P$ and $P_{1}$. The 
contour $C_{2}$ in polar coordinates takes the form

$$
s=-i k\left(z-z_{c}\right)+r e^{i \theta}, \pi<\theta<-\pi
$$

So,

$\int_{C_{2}} \frac{1}{s} \frac{\left(z-z_{c}-i s / k\right)^{1 / 2+i \gamma}}{\left(z_{1}-z_{c}-i s / k\right)^{1 / 2+i \gamma}} \quad e^{s t} d s=\int_{-\pi}^{\pi} \frac{\left.\left(-i r / k^{i \theta}\right) / k\right)^{1 / 2+i \gamma}}{\left.\left(z_{1}-z-i r / k e^{i \theta}\right)\right)^{1 / 2+i \gamma}} e^{\left(-i k\left(z-z_{c}\right)+r e^{i \theta}\right) t} i r e^{i \theta} d \theta$.

When $r \rightarrow 0$, the integral on the right-hand side of (A.15) has the order of $r^{3 / 2+i \gamma}$. The integral $\int_{C_{2}}$ converges to 0 when $r \rightarrow 0$. In a similar way, it can be proved that the integral along the contour surrounding the other branch point $s=-i k\left(z_{1}-z_{c}\right)+r e^{i \theta}$ converges to 0 when $r \rightarrow 0$. So, $\int_{C_{3}}=0$. The contours $C_{4}$ and $C_{5}$ are lines which can be written as $s=\rho e^{i \pi}-i k\left(z-z_{c}\right)$ and $s=\rho e^{-i \pi}-i k\left(z-z_{c}\right)$. Along the contour $C_{4}$ the variable $\rho$ runs from $\infty$ to 0 . Along the contour $C_{5}$ the variable $\rho$ goes in the opposite direction, from 0 to $\infty$. Thus

$$
\int_{C_{4}} \frac{1}{s} \frac{\left(z-z_{c}-i s / k\right)^{1 / 2+i \gamma}}{\left(z_{1}-z_{c}-i s / k\right)^{1 / 2+i \gamma}} \quad e^{s t} d s=\int_{\infty}^{0} \frac{e^{\left(\rho e^{i \pi}-i k\left(z-z_{c}\right)\right) t}}{-\rho-i k\left(z-z_{c}\right)}\left[\frac{\rho e^{i \pi}}{i k\left(z_{1}-z\right)-\rho}\right]^{1 / 2+i \gamma} e^{i \pi} d \rho
$$

and

$$
\int_{C_{5}} \frac{1}{s} \frac{\left(z-z_{c}-i s / k\right)^{1 / 2+i \gamma}}{\left(z_{1}-z_{c}-i s / k\right)^{1 / 2+i \gamma}} e^{s t} d s=\int_{0}^{\infty} \frac{e^{\left(\rho e^{i \pi}-i k\left(z-z_{c}\right)\right) t}}{-\rho-i k\left(z-z_{c}\right)}\left[\frac{\rho e^{-i \pi}}{i k\left(z_{1}-z\right)-\rho}\right]^{1 / 2+i \gamma} e^{-i \pi} d \rho
$$

The sum of the integrals along contours $C_{4}$ and $C_{5}$ is

$$
\int_{C_{4}}+\int_{C_{5}}=i \int_{0}^{\infty} \frac{e^{-\rho t} e^{-i k\left(z-z_{c}\right) t}}{-\rho-i k\left(z-z_{c}\right)} \frac{\rho^{1 / 2+i \gamma}}{\left[i k\left(z_{1}-z\right)-\rho\right]^{1 / 2+i \gamma}}\left(e^{-\pi \gamma}+e^{\pi \gamma}\right) d \rho
$$


Because of the term $e^{-\rho t}$, the main contribution to the integral comes from small $\rho$. To integrate the function on the right-hand side we will expand the denominator in powers of $\rho$ and with $\rho$ small we will be able to approximate the series by the first term.

$$
\int_{C_{4}}+\int_{C_{5}}=-\frac{e^{-i k\left(z-z_{c}\right) t}\left(e^{-\pi \gamma}+e^{\pi \gamma}\right)}{k\left(z-z_{c}\right)\left(i k\left(z-z_{1}\right)\right)^{1 / 2+i \gamma}} \int_{0}^{\infty} \frac{e^{-\rho t} \rho^{1 / 2+i \gamma}}{\left(1+\frac{\rho}{i k\left(z-z_{c}\right)}\right)\left(1-\frac{\rho}{i k\left(z-z_{1}\right)}\right)^{1 / 2+i \gamma}} d \rho
$$

Now we can rewrite the sum of the integrals in (A.19) in the form:

$$
\int_{C_{4}}+\int_{C_{5}}=\left(e^{-\pi \gamma}+e^{\pi \gamma}\right)\left(\frac{g_{1}(z, t)}{k^{3 / 2+i \gamma}} \int_{0}^{\infty} e^{-\rho t} \rho^{1 / 2+i \gamma} d \rho+\frac{g_{2}(z, t)}{k^{5 / 2+i \gamma}} \int_{0}^{\infty} e^{-\rho t} \rho^{3 / 2+i \gamma} d \rho+\ldots\right)
$$

where

$$
\begin{aligned}
f_{1}(z) & =-\frac{e^{-i k\left(z-z_{c}\right) t}}{\left(z-z_{c}\right)\left(i\left(z-z_{1}\right)\right)^{1 / 2+i \gamma}} \\
f_{2}(z) & =-\frac{\left[(3 / 2+i \gamma) z-(1 / 2+i \gamma) z_{c}-z_{1}\right]}{\left(z-z_{c}\right)^{2}\left(i\left(z-z_{1}\right)\right)^{3 / 2+i \gamma}} e^{-i k\left(z-z_{c}\right) t}
\end{aligned}
$$

To integrate (A.20) we use the definition of the gamma-function (Abramowitz and Stegun, 1964)

$$
\int_{0}^{\infty} e^{-\rho t} \rho^{a-1} d x=\Gamma(a) t^{-a}
$$

where $a$ is complex and the real part of $a$ is positive. So, we have

$$
\int_{C_{4}}+\int_{C_{5}}=2 i \pi e^{-i k\left(z-z_{c}\right) t}\left[\frac{G_{1}(z)}{t^{3 / 2+i \gamma}}+\frac{G_{2}(z)}{t^{5 / 2+i \gamma}}+O\left(t^{-7 / 2}\right)\right]
$$


In a similar way we can find the integrals along contours $C_{6}$ and $C_{7}$.

$$
\int_{C_{6}}+\int_{C_{7}}=2 i \pi e^{-i k\left(z_{1}-z_{c}\right) t}\left[\frac{G_{3}(z)}{t^{1 / 2-i \gamma}}+\frac{G_{4}(z)}{t^{3 / 2-i \gamma}}+O\left(t^{-5 / 2}\right)\right]
$$

where

$$
\begin{aligned}
& G_{1}(z)=-\frac{\left(e^{-\pi \gamma}+e^{\pi \gamma}\right) \Gamma(3 / 2+i \gamma)}{2 i \pi\left(z-z_{c}\right)\left(i\left(z-z_{1}\right)\right)^{1 / 2+i \gamma} k^{3 / 2+i \gamma}}, \\
& G_{2}(z)=-\frac{\left(e^{-\pi \gamma}+e^{\pi \gamma}\right) \Gamma(5 / 2+i \gamma)\left[(3 / 2+i \gamma) z-(1 / 2+i \gamma) z_{c}-z_{1}\right]}{2 i \pi\left(z-z_{c}\right)^{2}\left(i\left(z-z_{1}\right)\right)^{3 / 2+i \gamma} k^{5 / 2+i \gamma}}, \\
& G_{3}(z)=\frac{\left(e^{-\pi \gamma}+e^{\pi \gamma}\right) \Gamma(1 / 2-i \gamma)\left(i\left(z-z_{1}\right)\right)^{1 / 2+i \gamma}}{2 i \pi\left(z_{1}-z_{c}\right) k^{1 / 2-i \gamma}}, \\
& G_{4}(z)=-\frac{\left(e^{-\pi \gamma}+e^{\pi \gamma} \Gamma(3 / 2-i \gamma)\left(i\left(z-z_{1}\right)\right)^{-1 / 2+i \gamma}\right.}{2 i \pi\left(z_{1}-z_{c}\right) k^{3 / 2-i \gamma}}\left[z+z_{1}(1 / 2-i \gamma)+z_{c}(-1 / 2-i \gamma)\right] .
\end{aligned}
$$

The residue at the pole $s=0$ is

$$
\operatorname{Res}(s=0)=\lim _{s \rightarrow 0} \frac{\left(z-z_{c}-i s / k\right)^{1 / 2+i \gamma}}{\left(z_{1}-z_{c}-i s / k\right)^{1 / 2+i \gamma}} e^{s t}=\left(\frac{z-z_{c}}{z_{1}-z_{c}}\right)^{1 / 2+i \gamma} .
$$

So, now we can find $\phi(z, t)$

$$
\phi(z, t)=\left(\frac{z-z_{c}}{z_{1}-z_{c}}\right)^{1 / 2+i \gamma}-\frac{1}{2 \pi i}\left(\int_{C_{4}}+\int_{C_{5}}+\int_{C_{6}}+\int_{C_{7}}\right)
$$

So,

$$
\begin{aligned}
\phi(z, t)=\left(\frac{z-z_{c}}{z_{1}-z_{c}}\right)^{1 / 2+i \gamma} & -e^{-i k\left(z-z_{c}\right) t}\left[\frac{G_{1}(z)}{t^{3 / 2+i \gamma}}+\frac{G_{2}(z)}{t^{5 / 2+i \gamma}}\right] \\
& -e^{-i k\left(z_{1}-z_{c}\right) t}\left[\frac{G_{3}(z)}{t^{1 / 2-i \gamma}}+\frac{G_{4}(z)}{t^{3 / 2-i \gamma}}\right] .
\end{aligned}
$$




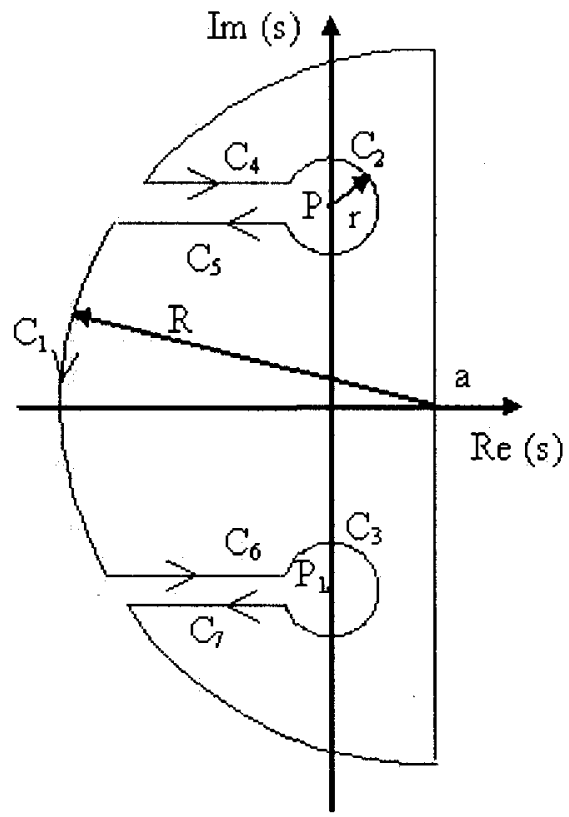

Figure A.1: Contour of integration in the complex plane for equation (A.8). 


\section{Appendix B}

In Appendix B we show how we used the inverse Laplace and Fourier transforms to get the solution of (5.8) of section 5.3 .

\section{Fourier transform}

The Fourier transform of a given function $\psi(x)$ is the function

$$
\tilde{\psi}(\lambda)=\mathcal{F}(\psi(x))=\int_{-\infty}^{\infty} \psi(x) e^{-i \lambda x} d x
$$

and the inverse Fourier transform is defined by

$$
\psi(x)=\mathcal{F}^{-1}(\tilde{\psi}(\lambda))=\frac{1}{2 \pi} \int_{-\infty}^{\infty} \tilde{\psi}(\lambda) e^{i \lambda x} d \lambda
$$

We find the function $\psi(x, z, t)$ by using the inverse Laplace and Fourier transforms:

$$
\psi(x, z, t)=\frac{1}{2 \pi i} \frac{1}{2 \pi} \int_{a-i \infty}^{a+i \infty} \frac{1}{s} e^{s t} \int_{-\infty}^{\infty} \tilde{A}(\lambda, \mu) e^{i \lambda x} F(\lambda, s) d \lambda d s
$$

where

$$
F(\lambda, s)=\frac{\left(z-z_{c}-i s / \lambda\right)^{1 / 2+i \gamma}}{\left(z_{1}-z_{c}-i s / \lambda\right)^{1 / 2+i \gamma}}
$$


and

$$
\tilde{A}(\lambda, \mu)=\frac{\sqrt{\pi}}{\mu} e^{-\frac{(\lambda-k)^{2}}{4 \mu^{2}}}
$$

where $a$ is a real number chosen so that the contour of integration lies to the right of the singularities of the integrand.

\section{Inverse Fourier transform}

The inversion of the Fourier transform is carried out first. Let $\tilde{\psi}(x, z, s)$ denote the inverse transform of $\tilde{\phi}(\lambda, z, s)$, then

$$
\tilde{\psi}(x, z, s)=\frac{1}{2 \pi s} \int_{-\infty}^{\infty} e^{i \lambda x} F(\lambda, s) d \lambda
$$

Since $\mu$ is small we can conclude that the dominant contribution to the above integral comes from the values $\lambda$ lying in a small neighbourhood around the point $\lambda=k$. We can expand $F(\lambda, s)$ in powers of $(\lambda-k)$, approximate it by the first two terms in the series and integrate with respect to $(\lambda-k)$. Then $\tilde{\psi}$ can be written as

$$
\begin{aligned}
\tilde{\psi}(x, z, s) \sim A(\mu x) \frac{e^{i k x}}{s}[ & {\left[F(k, s)+2 i \mu^{2} x \frac{\partial F}{\partial \lambda}(k, s)\right.} \\
& \left.+\mu^{2} \frac{\partial^{2} F}{\partial \lambda^{2}}(k, s)+O\left(\mu^{3}\right)\right] .
\end{aligned}
$$

So, to find $\psi$ we have to apply an inverse Laplace transform

$$
\begin{array}{r}
\psi(x, z, s)=A(\mu x) e^{i k x}\left\{\mathcal{L}^{-1}\left[\frac{F(k, s)}{s}\right]+2 i \mu^{2} x \mathcal{L}^{-1}\left[\frac{1}{s} \frac{\partial F}{\partial \lambda}(k, s)\right]\right. \\
\left.+\mu^{2} \mathcal{L}^{-1}\left[\frac{1}{s} \frac{\partial^{2} F(k, s)}{\partial \lambda^{2}}\right]+O\left(\mu^{3}\right)\right\}
\end{array}
$$


or

$$
\begin{array}{r}
\psi(x, z, s)=A(\mu x) e^{i k x}\left\{\mathcal{L}^{-1}\left[\frac{F(k, s)}{s}\right]-\frac{2 i \mu^{2} x}{k} \mathcal{L}^{-1}\left[\frac{\partial F}{\partial s}(k, s)\right]\right. \\
\left.+\frac{\mu^{2}}{k^{2}} \mathcal{L}^{-1}\left[\frac{\partial^{2}(s F(k, s))}{\partial s^{2}}\right]+O\left(\frac{\mu^{3}}{k^{3}}\right)\right\} .
\end{array}
$$

According to the properties of the Laplace transform

$$
\mathcal{L}^{-1}\left[\frac{\partial F(k, s)}{\partial s}\right]=-t f(t),
$$

where $f(t)=\mathcal{L}^{-1}[F]$, and

$$
\mathcal{L}^{-1}\left[\frac{\partial^{2}(s F(k, s))}{\partial s^{2}}\right]=t^{2} \mathcal{L}^{-1}[s F]
$$

As shown in Appendix A

$$
\begin{aligned}
\mathcal{L}^{-1}\left[\frac{F(k, s)}{s}\right]=\left(\frac{z-z_{c}}{z-z_{1}}\right)^{1 / 2+i \gamma} & -e^{-i k\left(z-z_{c}\right) t}\left[\frac{g_{1}(z)}{(k t)^{3 / 2+i \gamma}}+\frac{g_{2}(z)}{(k t)^{5 / 2+i \gamma}}\right] \\
& -e^{-i k\left(z_{1}-z_{c}\right) t}\left[\frac{g_{3}(z)}{(k t)^{1 / 2-i \gamma}}+\frac{g_{4}(z)}{(k t)^{3 / 2-i \gamma}}\right]
\end{aligned}
$$

where

$$
\begin{aligned}
& g_{1}=-\frac{\left(e^{\pi \gamma}+e^{-\pi \gamma}\right) \Gamma(3 / 2+i \gamma)}{2 \pi i\left(z-z_{c}\right)\left(i\left(z_{1}-z\right)\right)^{1 / 2+i \gamma}}, \\
& g_{2}=-\frac{\left(e^{\pi \gamma}+e^{-\pi \gamma}\right) \Gamma(5 / 2+i \gamma)}{2 \pi i\left(z-z_{c}\right)^{2}\left(i\left(z_{1}-z\right)\right)^{3 / 2+i \gamma}}\left[(3 / 2+i \gamma) z-(1 / 2+i \gamma) z_{c}-z_{1}\right], \\
& g_{3}=-\frac{\left(e^{\pi \gamma}+e^{-\pi \gamma}\right) \Gamma(1 / 2-i \gamma)\left(i\left(z_{1}-z\right)\right)^{1 / 2+i \gamma}}{2 \pi i\left(z_{1}-z_{c}\right)}, \quad \text { (B.13) } \\
& g_{4}=-\frac{\left(e^{\pi \gamma}+e^{-\pi \gamma}\right) \Gamma(3 / 2-i \gamma)\left(i\left(z_{1}-z\right)\right)^{-1 / 2+i \gamma}\left((1 / 2-i \gamma) z_{1}-(1 / 2+i \gamma) z_{c}+z\right)}{2 \pi i\left(z_{1}-z_{c}\right)} .
\end{aligned}
$$


To find $\mathcal{L}^{-1}[F]$ we integrate around the same contour shown in Figure A1. This time the integrand has no pole, only two branch points.

$$
\begin{aligned}
\mathcal{L}^{-1}[F]= & -e^{-i k\left(z-z_{c}\right) t}\left[\frac{g_{5}(z)}{t^{3 / 2+i \gamma} k^{1 / 2+i \gamma}}+\frac{g_{6}(z)}{t^{5 / 2+i \gamma} k^{3 / 2+i \gamma}}\right] \\
& e^{-i k\left(z_{1}-z_{c}\right) t}\left[\frac{h_{7}(z)}{t^{1 / 2-i \gamma} k^{-1 / 2-i \gamma}}+\frac{g_{8}(z)}{t^{3 / 2-i \gamma} k^{1 / 2-i \gamma}}\right]
\end{aligned}
$$

where

$$
\begin{aligned}
& g_{5}=\frac{\left(e^{\pi \gamma}+e^{-\pi \gamma}\right) \Gamma(3 / 2+i \gamma)}{2 \pi\left(i\left(z_{1}-z\right)\right)^{1 / 2+i \gamma}} \\
& g_{6}=\frac{\left(e^{\pi \gamma}+e^{-\pi \gamma}\right) \Gamma(5 / 2+i \gamma)(1 / 2+1 \gamma)}{2 \pi\left(i\left(z_{1}-z\right)\right)^{3 / 2+i \gamma}} \\
& g_{7}=\frac{1}{2 \pi}\left(e^{\pi \gamma}+e^{-\pi \gamma}\right) \Gamma(1 / 2-i \gamma)\left(i\left(z_{1}-z\right)\right)^{1 / 2+i \gamma} \\
& g_{8}=\frac{(1 / 2+i \gamma)\left(e^{\pi \gamma}+e^{-\pi \gamma}\right) \Gamma(3 / 2-i \gamma)}{2 \pi\left(i\left(z_{1}-z\right)\right)^{1 / 2+i \gamma}}
\end{aligned}
$$

$$
\begin{aligned}
\mathcal{L}^{-1}[s F] & =-e^{-i k\left(z-z_{c}\right) t}\left[\frac{g_{9}(z) k^{1 / 2-i \gamma}}{t^{3 / 2+i \gamma}}+\frac{g_{10}(z)}{t^{5 / 2+i \gamma} k^{1 / 2+i \gamma}}\right] \\
& -e^{-i k\left(z_{1}-z_{c}\right) t}\left[\frac{g_{11}(z) k^{3 / 2+i \gamma}}{t^{1 / 2-i \gamma}}+\frac{g_{12}(z) k^{1 / 2+i \gamma}}{t^{3 / 2-i \gamma}}\right]
\end{aligned}
$$

where

$$
\begin{aligned}
& g_{9}=-\frac{\left(e^{\pi \gamma}+e^{-\pi \gamma}\right) \Gamma(3 / 2+i \gamma)\left(z-z_{c}\right)}{2 \pi\left(z_{1}-z\right)^{1 / 2+i \gamma}} \\
& g_{10}=-\frac{(1 / 2+1 \gamma)\left(e^{\pi \gamma}+e^{-\pi \gamma}\right) \Gamma(5 / 2+i \gamma)}{2 \pi\left(z_{1}-z\right)}\left[z_{1}+(-1 / 2+i \gamma) z-(1 / 2+i \gamma) z_{c}\right] \\
& g_{11}=\frac{\left(e^{\pi \gamma}+e^{-\pi \gamma}\right) \Gamma(1 / 2-i \gamma)\left(z_{1}-z_{c}\right)}{2 \pi i}\left(i\left(z_{1}-z\right)\right)^{1 / 2+i \gamma}
\end{aligned}
$$




$$
g_{12}=\frac{\left(e^{\pi \gamma}+e^{-\pi \gamma}\right) \Gamma(3 / 2-i \gamma)}{2 \pi i\left(i\left(z_{1}-z\right)\right)^{1 / 2-i \gamma}}\left[z+(-3 / 2+i \gamma) z_{1}+(1 / 2+i \gamma) z_{c}\right]
$$

Having found $L^{-1}[F]$ and $\mathcal{L}^{-1}[s F]$ we can compute

$$
\mathcal{L}^{-1}\left[\frac{\partial F}{\partial s}\right]=-t \mathcal{L}^{-1}[F] \quad \text { and } \quad \mathcal{L}^{-1}\left[\frac{\partial^{2}(s F)}{\partial s^{2}}\right]=t^{2} \mathcal{L}^{-1}[s F]
$$

Finally, the inverse Laplace transform of the function $\tilde{\psi}$ is

$$
\begin{array}{r}
\psi=e^{i k x} A(\mu x)\left\{\left(\frac{z-z_{c}}{z_{1}-z_{c}}\right)^{1 / 2+i \gamma}-e^{-i k\left(z-z_{c}\right)}\left[\left(\frac{g_{1}}{(k t)^{3 / 2+i \gamma}}+\frac{g_{2}}{(k t)^{5 / 2+i \gamma}}\right)\right.\right. \\
\left.+\frac{2 \mu^{2} i x}{k}\left(g_{5}(k t)^{1 / 2+i \gamma}+\frac{g_{6}}{(k t)^{3 / 2+i \gamma}}\right)+\frac{\mu^{2}}{k^{2}}\left(g_{9}(k t)^{1 / 2-i \gamma}+\frac{g_{10}}{(k t)^{1 / 2+i \gamma}}\right)\right]( \\
-e^{-i k\left(z_{1}-z_{c}\right)}\left[\left(\frac{g_{3}}{(k t)^{1 / 2-i \gamma}}+\frac{g_{4}}{(k t)^{3 / 2-i \gamma}}\right)+\frac{2 \mu^{2} i x}{k}\left(g_{7}(t k)^{1 / 2-i \gamma}+\frac{g_{8}}{(k t)^{1 / 2-i \gamma}}\right)\right. \\
\left.\left.+\frac{\mu^{2}}{k^{2}}\left(g_{11}(k t)^{3 / 2+i \gamma}+g_{12}(k t)^{1 / 2+i \gamma}\right)\right]\right\}
\end{array}
$$




\section{Appendix C}

\section{Asymptotic and multiple scale analysis}

Multiple scale analysis (see, e.g. Bender and Orszag (1978)) is a very general collection of perturbation techniques. It is particularly useful for constructing valid approximation to solutions of perturbation problems. Let us consider the oscillation equation

$$
u_{t t}+u+\varepsilon u^{3}=0
$$

If we use perturbation theory to solve this equation we get secular terms and can not get a bounded solution. To eliminate secular terms in multiple-scale analysis we introduce a new variable $\tau=\varepsilon t$. $\tau$ defines a long time scale because $\tau$ is not negligible when $t$ is of order $1 / \varepsilon$ or larger. Even though the exact solution $u(t)$ is a function of $t$ alone, multiple-scale analysis seeks solutions which are functions of both variables $t$ and $\tau$ treated as independent variables. The formal procedure consists of the following steps: 
- Assume a solution of the form

$$
u(t)=u_{0}(t, \tau)+\varepsilon u_{1}(t, \tau)+O\left(\varepsilon^{2}\right)
$$

- Use the chain rule to compute the derivatives of $u(t, \tau)$, e.g.

$$
\frac{d u}{d t}=\frac{\partial u_{0}}{\partial t}+\varepsilon\left(\frac{\partial u_{0}}{\partial \tau}+\frac{\partial u_{1}}{\partial t}\right)+O\left(\varepsilon^{2}\right)
$$

- Substitute these derivatives into the differential equation and group terms of $O(1), O(\varepsilon), O\left(\varepsilon^{2}\right)$ and so on. This gives a series of equations which are solved. This technique is used in Chapters 5 and 6 in the thesis. 


\section{Appendix D}

\section{The Eliassen-Palm theorem and the mean flow evolution}

\section{D.1 Momentum flux and mean flow}

In section 4.3 we showed that in the linear problem the wave amplitude is reduced by a factor of $e^{-\gamma \pi}$, where $\gamma=\sqrt{N^{2}-1 / 4}$, across the critical level. Since $N^{2}>>1 / 4, \gamma$ is large and so the wave amplitude is reduced to close to zero above the critical level.

In the nonlinear problem any change in the wave amplitude at any level results in a change in the mean flow. The mean flow velocity $\bar{u}$ defined to be the horizontal average of the initial total horizontal velocity $\bar{u}=-\overline{\Psi_{z}(x, z, 0)}$. In a case with a monochromatic forcing the average is taken over a wavelength, so the overbar denotes:

$$
\overline{(\ldots)}=\frac{k}{2 \pi} \int_{0}^{2 \pi / k}(\ldots) d x
$$

In a case with a wave packet forcing the average is taken over the horizontal extent of the forcing. Let us call this length $L$ which is defined so that everything is zero at 
$x=-L / 2$ and $x=L / 2$.

$$
\bar{u}(z)=-\bar{\Psi}_{z}(z)=\frac{1}{L} \int_{-L / 2}^{L / 2} \Psi(x, z, 0) d x
$$

We will denote the average $\frac{1}{L} \int_{-L / 2}^{L / 2}(\ldots) d x$ by an overbar.

In the nonlinear problem the mean flow changes with time because of interaction between waves with positive and negative wavenumbers i.e. terms of the form $e^{i k x}$ and $e^{-i k x}$ which when multiplied together give terms of the form $e^{0}$ and $e^{2 i k x}$. The zero wavenumber terms represent changes in the mean flow. We can obtain an equation that describes the evolution of the mean flow by taking the mean $\frac{1}{L} \int_{-L / 2}^{L / 2}(\ldots) d x$ of each term in our original linearized equation for the total variables $(u, w, \rho, p)$.

$$
\frac{\partial u}{\partial t}+u \frac{\partial u}{\partial x}+w \frac{\partial u}{\partial z}+\frac{1}{\rho} \frac{\partial p}{\partial x}=0
$$

where $u=-\Psi_{z}$ and $w=\Psi_{x}$.

In terms of the total streamfunction this equation can be written as

$$
\frac{\partial \Psi_{z}}{\partial t}-\Psi_{z} \Psi_{z x}+\Psi_{x} \Psi_{z z}+\frac{1}{\rho} \frac{\partial p}{\partial x}=0
$$

After averaging we have

$$
\overline{\frac{\partial \Psi_{z}}{\partial t}}=\overline{\Psi_{z} \Psi_{z x}-\Psi_{x} \Psi_{z z}}
$$

Substituting $\Psi(x, z, t)=\bar{\psi}+\varepsilon \psi(x, z, t)$ gives

$$
\overline{\frac{\partial \Psi_{z}}{\partial t}}=\varepsilon^{2} \overline{\Psi_{z} \Psi_{z x}-\Psi_{x} \Psi_{z z}}
$$


The first term on the right-hand side is

$$
\frac{1}{L} \int_{-L / 2}^{L / 2} \psi_{z} \psi_{x z} d x=\left.\frac{1}{2 L} \psi_{z}^{2}\right|_{-L / 2} ^{L / 2}=0
$$

So, we can subtract two times this zero term from the right-hand side to obtain

$$
\frac{\overline{\partial \Psi_{z}}}{\partial t}=\varepsilon^{2} \overline{-\psi_{z} \psi_{z x}-\psi_{x} \psi_{z z}}=-\varepsilon^{2} \frac{\partial}{\partial z} \overline{\psi_{x} \psi_{z}}
$$

The left-hand side is the rate of change in the mean flow with time as a result of the nonlinear interactions with $\bar{u}=-\overline{\Psi_{z}(x, z, 0)}$ being the initial mean flow. Let us denote the change in the mean flow at time $t$ as $\Delta \bar{u}(z, t)$. So the total mean flow at time $t$ is $\Psi_{z}=\bar{u}+\Delta \bar{u}(z, t)$. The term $\left(-\psi_{x} \psi_{z}\right)$ is the vertical flux of horizontal momentum $F$, and $F=-\overline{\psi_{x} \psi_{z}}$ is its horizontal average. This is also called the Reynold's stress. So equation (D.7) can be written as

$$
\frac{\partial \Delta \bar{u}}{\partial t}=\varepsilon^{2} \frac{\partial F}{\partial z}
$$

The Eliassen-Palm theorem states that for linear waves $F$ is independent of height in the absence of singularities. If there is a critical level, i.e. a singularity in the linear problem, $F$ is discontinuous at the critical level. At the critical layer where the wave amplitude changes by a factor of $e^{-\gamma \pi}$ (as was shown in section 4.3) the Reynold's stress $F$ changes by a factor of $e^{-2 \gamma \pi}$. In the nonlinear problem such a change in $F$ with height means that according to (D.8) there is a change $\bar{u}$ in the mean flow near the critical level. In our problem $F$ is negative below the critical level and it goes to zero above. So the jump in $F,[F]=F_{\text {above }}-F_{\text {below }}$, is positive and there is a positive change in the mean velocity, i.e. an acceleration in the mean flow. The physical interpretation of this is that the wave are absorbed by the mean flow at the 
critical level, or that the waves deposit momentum in the mean flow at the critical level.

\section{D.2 The Eliassen-Palm theorem}

\section{The Eliassen-Palm theorem:}

For linear waves the momentum flux $F$ is independent of height in the absence of singularities.

\section{Proof:}

We consider the linear problem and assume the waves are time-independent and periodic in $x, z$ and $t$

$$
\psi=C e^{i(k x+m z-\omega t)}+\text { c.c. }=C e^{i(k x+m z-\omega t)}+C^{*} e^{-i(k x+m z-\omega t)}
$$

We find the averaged momentum flux which is $F=-\overline{\psi_{x} \psi_{z}}$ :

$\psi_{x} \psi_{z}=\left(i k C e^{i(k x+m z-\omega t)}-i k C^{*} e^{-i(k x+m z-\omega t)}\right) \cdot\left(i m C e^{i(k x+m z-\omega t)}-i m C^{*} e^{-i(k x+m z-\omega t)}\right)$

So,

$$
\psi_{x} \psi_{z}=-k m\left(C^{2} e^{2 i(k x+m z-\omega t)}+C^{* 2} e^{-2 i(k x+m z-\omega t)}-2 C C^{*}\right)
$$

The averaged momentum flux is $F=-\overline{\psi_{x} \psi_{z}}$. Averaging the expression in the righthand side of (D.11) over an $x$-wavelength $2 \pi / k$ we get $\overline{\psi_{x} \psi_{z}}=2 k m|C|^{2}$. So, the momentum flux is

$$
F=-\overline{\psi_{x} \psi_{z}}=-2 k m|C|^{2}
$$

$F$ is constant, and for positive $m$ and $k$ it is is negative. 


\section{Remark:}

For the time-dependent case we consider the time-dependent wave

$$
\psi=\phi(z, t) e^{i k x}+\text { c.c. }=\phi(z, t) e^{i k x}+\phi^{*}(z, t) e^{-i k x}
$$

After the same procedure as for the steady case, we find that the momentum flux is

$$
F=-\overline{\psi_{x} \psi_{z}}=i k \phi^{*} \phi_{z}-i k \phi_{z}^{*} \phi=-2 k \operatorname{Im}\left(\phi \phi^{*}\right)
$$

In the absence of a critical layer, $F$ is approximately independent on $z$ if the wave amplitude is oscillatory in the $z$ direction, i.e. if $\phi \sim e^{i m z}$. If $k$ and $m$ are positive, the momentum flux is negative.

In problems with critical layers the momentum flux is discontinuous across the critical layer and it goes to zero at the critical layer. So there is a positive jump in $F$ across the critical layer.

In the nonlinear problem, at early time the solution behaves like the solution of the linear problem. So, at early time there is a positive jump in $F$ across the critical layer. According to equation (D.8) the mean flow is accelerated near the critical layer as a result of the wave absorption. Numerical solutions show that at late time the jump in $F$ goes to zero. This indicates that there is wave reflection, i.e. momentum being transferred back to the mean flow. 


\section{Bibliography}

[1] Abramowitz, M., Stegun, I.A., 1964, Handbook of mathematical functions with formulas, graphs and mathematical tables, Nat. Bur. Stands, 1046 pp.

[2] Bacmeister, J.T., Pierrehumbert, R.T., 1988, On high-drag states of nonlinear stratified flow over an obstacle, J. Atmos. Sci., 45, 63-80.

[3] Bailey, D.H., Swarztrauber, P.N., 1991, The fractional Fourier transform and applications, SIAM Review, 33, 389-404.

[4] Bailey, D.H., Swarztrauber, P.N., 1994, A fast method for the numerical evaluation of continuous Fourier and Laplace transforms, J. Sci. Comput., 15, 11051110.

[5] Baines, P., 1995, Topographic effects in stratified flows, Cambridge University Press, 482 pp.

[6] Batchelor, G.K., 1967, An introduction to fluid dynamics, Cambridge University Press.

[7] Bender C.M., Orszag S.A. 1978, Advanced mathematical methods for scientists and engeneers, McGraw-Hill, Inc., 593 pp.

[8] Booker, J.R., Bretherton, F.P., 1967, The critical layer for gravity waves in a shear flow, J. Fluid Mech., 27, 513-539. 
[9] Brown, S.N., Stewartson, K., 1978, The evolution of the critical layer of a Rossby wave, Part 2, Geophys. Astrophys. Fluid Dyn., 10, 1-24.

[10] Brown, S.N., Stewartson, K., 1982, On the nonlinear reflection of a gravity wave at a critical level, Part 3, J. Fluid Mech., 115, 231-250.

[11] Campbell, L.J., Maslowe, S.A., 1998, Forced Rossby wave packets in barotropic shear flows with critical layers, Dyn. Atmos. Oceans, 28, 9-37.

[12] Campbell, L.J., Maslowe, S.A., 2001, A numerical simulation of the nonlinear critical layer evolution of a forced Rossby wave packet in a zonal shear flow, Mathematics and Computers in Simulation, 55, 365-375.

[13] Campbell, L.J., Maslowe, S.A., 2003, Nonlinear critical-layer evolution of a forced gravity wave packet, J.Fluid Mech., 493, 151-179.

[14] Fritts, D.C., 1982, The transient critical level interaction in a Boussinesq fluid, J. Geophys. Res., 87, 7997-8016.

[15] Goldstein, S., 1931, On the stability of superposed streams of fluids of different densities, Proc. R. Soc. London A, 132, 524-548.

[16] Haynes, P.H., 1985, Nonlinear instability of a Rossby wave critical layer, $J$. Fluid Mech., 161, 493-511.

[17] Howard, L.N., 1961, Note on a paper of John W. Miles, J. Fluid Mech., 10, $509-512$.

[18] Kundu, P.K., Cohen, I.M., 2004, Fluid Mechanics, third edition, Elsevier Academic Press, 759 pp. 
[19] Landau, L.D., Lifshitz,E.M., 1953, Course of theoretical physics, Fluid Mechanics, Nauka, 630 pp.

[20] Miles, J.W., 1961, On the stability of heterogenous flows, J. Fluid Mech., 10, 496-508.

[21] Nappo, C.J., 2002, An introduction to atmospheric gravity waves, Elsevier, 276 pp.

[22] Spiegel, E.A., Veronis, G., 1960, On the Boussinesq approximation for a compressible fluid, Astrophys. J., 131, 442-447.

[23] Taylor, G.I., 1931, Effect of variation in density on the stability of superposed streams of fluids, Proc. R. Soc. London A, 201, 499-523. 